Supporting Information

\title{
AuCl-Catalyzed Synthesis of Benzyl-Protected Substituted Phenols: A Formal [3+3] approach
}

\author{
Xiaogen Huang and Liming Zhang* \\ Department of Chemistry/216, University of Nevada, Reno \\ 1664 North Virginia Street, Reno, Nevada 89557
}

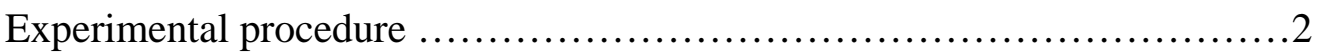

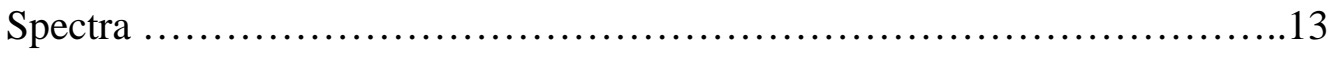


General: Ethyl acetate (ACS grade), hexanes (ACS grade) and diethyl ether (ACS grade) were purchased from Fisher Scientific and used without further purification. Anhydrous tetrahydrofuran and anhydrous dichloromethane in Pure-Pac ${ }^{\mathrm{TM}}$ from Aldrich were used directly without further treatment. Commercially available reagents were used without further purification. The NMR data were the same as reported. Reactions were monitored by thin layer chromatography (TLC) using silicycle precoated silica gel plates. Flash column chromatography was performed over silicycle silica gel (230-400 mesh). ${ }^{1} \mathrm{H}$ NMR and ${ }^{13} \mathrm{C}$ NMR spectra were recorded on a Varian $500 \mathrm{MHz}$ Unity plus spectrometer and a Varian $400 \mathrm{MHz}$ spectrometer using residue solvent peaks as internal standards. Infrared spectra were recorded with a Perkin Elmer FT-IR spectrum 2000 spectrometer and are reported in reciprocal centimeter $\left(\mathrm{cm}^{-1}\right)$. Mass spectra were recorded with Waters micromass $Z Q$ detector using electron spray method.

\section{General procedure A: Preparation of acetates from enals:}

To a solution of 1-benzyloxy-1,2-propadiene (161 mg, $1.1 \mathrm{mmol}, 1.1$ eq.) in $10 \mathrm{~mL}$ of THF cooled in a dry ice-acetone bath was added BuLi $(0.75 \mathrm{~mL}, 1.2 \mathrm{mmol}, 1.2$ eq.) dropwise under $\mathrm{N}_{2}$. The mixture was stirred for $1 \mathrm{~h}$ and TMEDA (0.2 mL, $1.2 \mathrm{mmol}, 1.2 \mathrm{eq}$.) was then introduced. After $15 \mathrm{~min}$, the appropriate enal $(1 \mathrm{mmol}, 1$ eq.) was added dropwise, and the reaction mixture was stirred for another $2 \mathrm{~h}$ at the same temperature before dropwise addition of $\mathrm{Ac}_{2} \mathrm{O}(0.25 \mathrm{~mL}$, $1.3 \mathrm{mmol}, 1.3$ eq.). The resulting mixture was allowed to warm to room temperature and then quenched with water. The mixture was extracted with ether $(3 \times 50 \mathrm{~mL})$, and the organic layers were combined and washed with brine. The residue obtained after solvent removal was purified through silica gel flash column chromatography ( $1 \mathrm{v} / \mathrm{v} \%$ of $\mathrm{Et}_{3} \mathrm{~N}$ was added to basify the silica gel) to offer the desired allenyl alkenyl carbinol acetate as a viscous liquid.

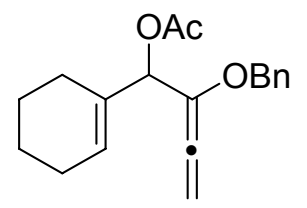

1

Compound 1 was isolated in $80 \%$ yield following the general procedure A using cyclohex 1 enecarboxaldehyde as the enal starting material. ${ }^{1} \mathrm{H}$ NMR $\left(400 \mathrm{MHz}, \mathrm{CDCl}_{3}\right)$ ס: 7.35-7.25 (m, $5 \mathrm{H}), 5.81(\mathrm{~s}, 1 \mathrm{H}), 5.65(\mathrm{~s}, 1 \mathrm{H}), 5.52-5.47(\mathrm{~m}, 2 \mathrm{H}), 4.68-4.62(\mathrm{~m}, 2 \mathrm{H}), 2.10-1.92(\mathrm{~m}, 7 \mathrm{H}), 1.64-$ $1.54(\mathrm{~m}, 4 \mathrm{H}) ;{ }^{13} \mathrm{C}$ NMR $\left(100.5 \mathrm{MHz}, \mathrm{CDCl}_{3}\right) \delta:$ 198.8, 169.9, 137.5, 133.3, 131.1, 128.2, 127.6, 127.4, 126.1, 92.1, 75.8, 70.5, 25.1, 24.7, 22.5, 22.2, 21.1; IR (neat): 2931, 2253, 1733, 1607, 1455, 1371, 1238, 1169, 1027, 908, 824, 731; MS $\left(E S^{+}\right)$Calculated for $\left[\mathrm{C}_{19} \mathrm{H}_{22} \mathrm{NaO}_{3}\right]^{+} 321.1$; Found: 321.1. 


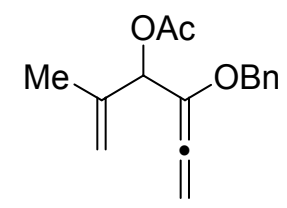

$3 a$

Compound $3 \mathrm{a}$ was isolated in $72 \%$ yield following the general procedure $\mathrm{A}$ using methacrolein as the enal starting material. ${ }^{1} \mathrm{H}$ NMR $\left(500 \mathrm{MHz}, \mathrm{CDCl}_{3}\right) \delta: 7.35-7.25(\mathrm{~m}, 5 \mathrm{H}), 5.72(\mathrm{~s}, 1 \mathrm{H})$, 5.54-5.49 (m, 2H), $5.08(\mathrm{~s}, 1 \mathrm{H}), 4.99(\mathrm{~s}, 1 \mathrm{H}), 4.66(\mathrm{~s}, 2 \mathrm{H}), 2.10(\mathrm{~d}, 3 \mathrm{H}, \mathrm{J}=1.5 \mathrm{~Hz}), 1.75(\mathrm{~s}, 3 \mathrm{H})$;

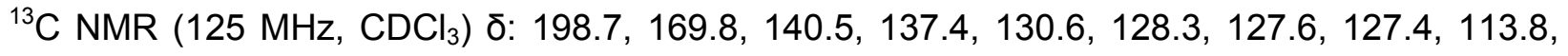
92.1, 75.5, 70.6, 21.1, 18.8; IR (neat): 2925, 1960, 1746, 1656, 1608, 1496, 1455, 1371, 1232 , 1172, 1028, 900, 824, 736; MS (ES ${ }^{+}$) Calculated for $\left[\mathrm{C}_{16} \mathrm{H}_{18} \mathrm{NaO}_{3}\right]^{+} 281.1$; Found: 281.1.

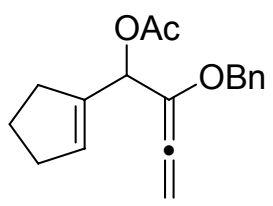

3b

Compound $\mathbf{3 b}$ was isolated in $53 \%$ yield following the general procedure $A$ using cyclopent-1enecarbaldehyde as the enal starting material. ${ }^{1} \mathrm{H} \mathrm{NMR}\left(500 \mathrm{MHz}, \mathrm{CDCl}_{3}\right) \delta: 7.35-7.25(\mathrm{~m}, 5 \mathrm{H})$, $5.92(\mathrm{~s}, 1 \mathrm{H}), 5.74(\mathrm{~s}, 1 \mathrm{H}), 5.51(\mathrm{~d}, 1 \mathrm{H}, \mathrm{J}=8.5 \mathrm{~Hz}), 5.49(\mathrm{~d}, 1 \mathrm{H}, \mathrm{J}=8.5 \mathrm{~Hz}), 4.66(\mathrm{~s}, 1 \mathrm{H}), 2.36-$

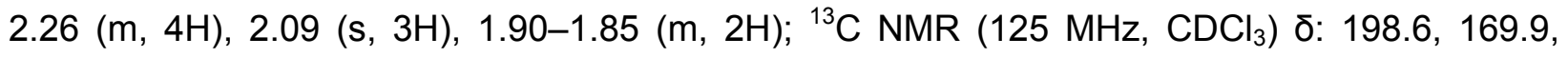
139.7, 137.4, 130.9, 128.9, 128.2, 127.6, 127.5, 92.1, 71.9, 70.6, 32.4, 32.2, 23.3, 21.1; IR (neat): $2253,1735,1607,1494,1455,1240,1049,907,735$; MS $\left(E^{+}\right)$Calculated for $\left[\mathrm{C}_{18} \mathrm{H}_{20} \mathrm{NaO}_{3}\right]^{+}$307.1; Found: 307.1 .

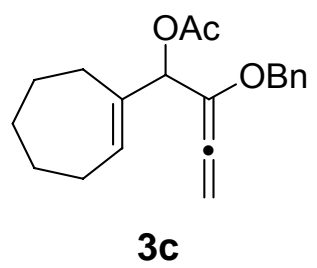

Compound $3 \mathrm{c}$ was isolated in $55 \%$ yield following the general procedure A using cyclohept-1enecarbaldehyde as the enal starting material. ${ }^{1} \mathrm{H} \mathrm{NMR}\left(500 \mathrm{MHz}, \mathrm{CDCl}_{3}\right) \delta: 7.35-7.25(\mathrm{~m}, 5 \mathrm{H})$, $5.95(\mathrm{t}, 1 \mathrm{H}, \mathrm{J}=6.5 \mathrm{~Hz}), 5.66(\mathrm{~s}, 1 \mathrm{H}), 5.51(\mathrm{~s}, 2 \mathrm{H}), 4.65(\mathrm{~d}, 1 \mathrm{H}, \mathrm{J}=12.0 \mathrm{~Hz}), 4.61(\mathrm{~d}, 1 \mathrm{H}, J=$ $12.0 \mathrm{~Hz}), 2.19-2.20(\mathrm{~m}, 4 \mathrm{H}), 2.06(\mathrm{~s}, 3 \mathrm{H}), 1.74-1.69(\mathrm{~m}, 2 \mathrm{H}), 1.55-1.39(\mathrm{~m}, 2 \mathrm{H}) ;{ }^{13} \mathrm{C}$ NMR $(125$ $\left.\mathrm{MHz}, \mathrm{CDCl}_{3}\right) \delta: 198.8,169.8,139.2,137.4,131.5,131.3,128.2,127.6,127.4,92.2,76.9,70.5$, 32.4, 28.9, 28.3, 26.7, 26.6, 21.2; IR (neat): 2253, 1735, 1607, 1494, 1455, 1370, 1240, 1168, 1028, 909, 732; MS (ES $\left.{ }^{+}\right)$Calculated for $\left[\mathrm{C}_{20} \mathrm{H}_{24} \mathrm{NaO}_{3}\right]^{+} 335.2$; Found: 335.1 . 


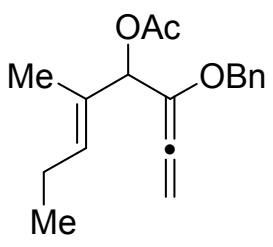

3d

Compound $\mathbf{3 d}$ was isolated in $70 \%$ yield following the general procedure A using 2-methylpent2-enal as the enal starting material. ${ }^{1} \mathrm{H}$ NMR $\left(500 \mathrm{MHz}, \mathrm{CDCl}_{3}\right) \delta: 7.35-7.25(\mathrm{~m}, 5 \mathrm{H}), 5.68(\mathrm{~s}$, $1 \mathrm{H}), 5.55(\mathrm{dt}, 1 \mathrm{H}, \mathrm{J}=1.0,7.0 \mathrm{~Hz}), 5.52-5.48(\mathrm{~m}, 2 \mathrm{H}), 4.66(\mathrm{~d}, 1 \mathrm{H}, \mathrm{J}=12.5 \mathrm{~Hz}), 4.62(\mathrm{~d}, 1 \mathrm{H}, \mathrm{J}=$ $12.5 \mathrm{~Hz}), 2.10-2.02(\mathrm{~m}, 5 \mathrm{H}), 1.61(\mathrm{~s}, 3 \mathrm{H}), 0.97(\mathrm{t}, 3 \mathrm{H}, \mathrm{J}=7.0 \mathrm{~Hz}) ;{ }^{13} \mathrm{C} \mathrm{NMR}\left(125 \mathrm{MHz}, \mathrm{CDCl}_{3}\right) \delta$ : 198.7, 169.9, 137.5, 131.6, 131.2, 129.8, 128.2, 127.6, 127.4, 92.2, 76.8, 70.5, 21.1, 21.0, 13.7, 12.5; IR (neat): 2251, 1607, 1500, 1454, 1380, 1288, 1230, 1199, 1164, 1123, 1048, 908, 730; MS (ES $\left.{ }^{+}\right)$Calculated for $\left[\mathrm{C}_{18} \mathrm{H}_{22} \mathrm{NaO}_{3}\right]^{+}$309.2; Found: 309.1 .

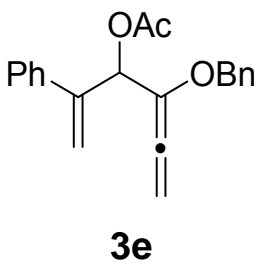

Compound $3 \mathrm{e}$ was isolated in $41 \%$ yield following the general procedure $\mathrm{A}$ using 2phenylpropenal as the enal starting material. ${ }^{1} \mathrm{H} \mathrm{NMR}\left(400 \mathrm{MHz}, \mathrm{CDCl}_{3}\right) \delta: 7.45-7.25(\mathrm{~m}, 10 \mathrm{H})$, $6.31(\mathrm{~s}, 1 \mathrm{H}), 5.46(\mathrm{~s}, 1 \mathrm{H}), 5.42-5.41(\mathrm{~m}, 3 \mathrm{H}), 4.58(\mathrm{~s}, 2 \mathrm{H}), 2.12(\mathrm{~s}, 3 \mathrm{H}) ;{ }^{13} \mathrm{C} \mathrm{NMR}(125 \mathrm{MHz}$, $\left.\mathrm{CDCl}_{3}\right)$ $\delta: 199.5,169.8,144.4,138.9,137.2,130.8,128.2,128.1,127.7,127.6,127.4,126.8$, 115.4, 92.2, 73.8, 70.9, 21.1; IR (neat): 2253, 1735, 1607, 1494, 1451, 1384, 1235, 1049, 907 , 730; $\mathrm{MS}\left(\mathrm{ES}^{+}\right)$Calculated for $\left[\mathrm{C}_{21} \mathrm{H}_{20} \mathrm{NaO}_{3}\right]^{+} 343.1$; Found: 342.9 .

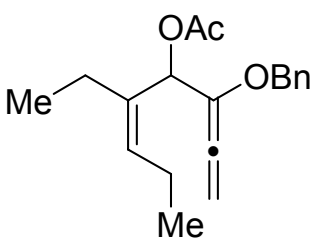

$3 f$

Compound $\mathbf{3 f}$ was isolated in $80 \%$ yield following the general procedure $A$ using $(Z)$-2-ethylpent2-enal as the enal starting material. ${ }^{1} \mathrm{H}$ NMR $\left(500 \mathrm{MHz}, \mathrm{CDCl}_{3}\right) \delta: 7.37-7.26(\mathrm{~m}, 5 \mathrm{H}), 6.22(\mathrm{t}, 1 \mathrm{H}$, $\mathrm{J}=2.5 \mathrm{~Hz}$ ), $5.50(\mathrm{~d}, 2 \mathrm{H}, \mathrm{J}=2.5 \mathrm{~Hz}$ ), $5.27(\mathrm{t}, 1 \mathrm{H}, \mathrm{J}=7.0 \mathrm{~Hz}$ ), 4.62 (q, 2H, J = $12.5 \mathrm{~Hz}), 2.22-$ $2.12(\mathrm{~m}, 2 \mathrm{H}), 2.10-2.06(\mathrm{~m}, 5 \mathrm{H}), 1.01(\mathrm{t}, 3 \mathrm{H}, \mathrm{J}=7.5 \mathrm{~Hz}), 0.94(\mathrm{t}, 3 \mathrm{H}, \mathrm{J}=7.5 \mathrm{~Hz}) ;{ }^{13} \mathrm{C}$ NMR $(125$ $\left.\mathrm{MHz}_{\mathrm{CDCl}}\right) \mathrm{CD}_{3} 198.4,169.9,137.5,135.3,131.8,131.1,128.2,127.5,127.2,92.4,70.53$, 
70.50, 24.6, 21.2, 21.1, 14.3, 12.9; IR (neat): 2965, 1960, 1744, 1495, 1455, 1370, 1233, 1173, 1044, 892; MS $\left(\mathrm{ES}^{+}\right)$Calculated for $\left[\mathrm{C}_{19} \mathrm{H}_{24} \mathrm{NaO}_{3}\right]^{+}$323.2; Found: 323.0.

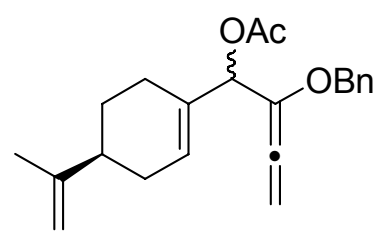

$3 g$

Compound $\mathbf{3 g}$ was isolated in $80 \%$ yield following the general procedure $A$ using (-)perillaldehyde as the enal starting material. ${ }^{1} \mathrm{H} \mathrm{NMR}\left(500 \mathrm{MHz}, \mathrm{CDCl}_{3}\right) \delta: 7.37-7.25(\mathrm{~m}, 5 \mathrm{H})$, 5.84-5.81 (m, 1H), $5.69(\mathrm{~d}, 1 \mathrm{H}, \mathrm{J}=5.5 \mathrm{~Hz}), 5.53-5.48(\mathrm{~m}, 2 \mathrm{H}), 4.71(\mathrm{~d}, 2 \mathrm{H}, \mathrm{J}=7.0 \mathrm{~Hz}), 4.68-$ $4.63(\mathrm{~m}, 2 \mathrm{H}), 2.12-1.94(\mathrm{~m}, 8 \mathrm{H}), 1.82-1.79(\mathrm{~m}, 1 \mathrm{H}), 1.73(\mathrm{~s}, 3 \mathrm{H}), 1.48-1.40(\mathrm{~m}, 1 \mathrm{H}) ;{ }^{13} \mathrm{C} \mathrm{NMR}$ $\left(125 \mathrm{MHz}, \mathrm{CDCl}_{3}\right)$ $\delta:$ 198.67, 198.63, 169.87, 169.85, 149.65, 149.63, 137.39, 137.38, 133.17, 132.88, 130.92, 130.86, 128.19, 127.60, 127.43, 127.42, 125.72, 125.40, 108.69, 108.67, 92.17, $92.16,75.54,75.34,70.49,70.48,40.82$, 40.78, 30.51, 30.44, 27.32, 27.31, 25.16, 25.14, 21.14, 21.11, 20.72, 20.69; IR (neat): 2926, 2253, 1734, 1607, 1494, 1452, 1372, 1240, 1049, 907, 824, 731; $\mathrm{MS}\left(\mathrm{ES}^{+}\right)$Calculated for $\left[\mathrm{C}_{22} \mathrm{H}_{26} \mathrm{NaO}_{3}\right]^{+} 361.2$; Found: 361.0 .

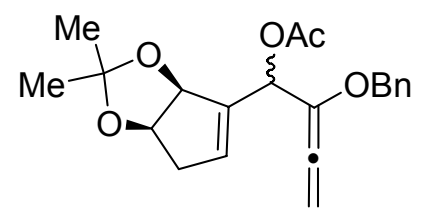

3h

Compound $3 \mathrm{~h}$ was isolated in $76 \%$ yield following the general procedure A using 2,2-dimethyl6,6a-dihydro-3aH-cyclopenta[1,3]dioxole-4-carbaldehyde as the enal starting material. ${ }^{1} \mathrm{H}$ NMR $\left(500 \mathrm{MHz}, \mathrm{CDCl}_{3}\right)$ ס: 7.37-7.22 (m, 5H), 6.07 (s, 0.4H), $5.94(\mathrm{~s}, 0.6 \mathrm{H}), 5.83(\mathrm{~s}, 0.6 \mathrm{H}), 5.72(\mathrm{~s}$, $0.4 \mathrm{H}), 5.57-5.51(\mathrm{~m}, 2 \mathrm{H}), 5.11(\mathrm{~d}, 0.6 \mathrm{H}, \mathrm{J}=5.5 \mathrm{~Hz}), 4.90(\mathrm{~d}, 0.4 \mathrm{H}, \mathrm{J}=6.0 \mathrm{~Hz}), 4.75(\mathrm{t}, 0.6 \mathrm{H}, \mathrm{J}$ $=5.5 \mathrm{~Hz}), 4.72(\mathrm{t}, 0.4 \mathrm{H}, \mathrm{J}=5.5 \mathrm{~Hz}), 4.69-4.63(\mathrm{~m}, 2 \mathrm{H}), 2.60-2.48(\mathrm{~m}, 2 \mathrm{H}), 2.12(\mathrm{~s}, 1.2 \mathrm{H}), 2.09$ $(\mathrm{s}, 1.8 \mathrm{H}), 1.39(\mathrm{~s}, 1.2 \mathrm{H}), 1.35(\mathrm{~s}, 1.8 \mathrm{H}), 1.33(\mathrm{~s}, 3 \mathrm{H}) ;{ }^{13} \mathrm{C} \mathrm{NMR}\left(125 \mathrm{MHz}, \mathrm{CDCl}_{3}\right) \delta: 199.03$, $198.67,169.89,169.57,139.61,139.21,137.29,137.22,131.22,130.63,129.71,128.26$, $128.25,127.72,127.68,127.62,127.55,127.48,110.50,110.24,92.45,92.08,85.02$, 84.76, 78.50, 78.26, 70.69, 70.59, 70.58, 70.55, 69.76, 37.89, 37.69, 27.51, 27.36, 26.28, 26.04, 21.11, 21.08; IR (neat): 1607, 1494, 1451, 1050, 907, 824, 731; MS (ES $)$ Calculated for $\left[\mathrm{C}_{21} \mathrm{H}_{24} \mathrm{NaO}_{5}\right]^{+}$ 379.2; Found: 378.8.

General procedure B: AuCl-catalyzed formation of benzyl-protected substituted phenols from purified alkenyl allenyl carbinol acetates 
To a solution of an acetate (1, 3a-h, $0.1 \mathrm{mmol}, 1$ eq.) in $2 \mathrm{~mL}$ of $\mathrm{CH}_{2} \mathrm{Cl}_{2}$ was added $\mathrm{AuCl}$ (0.005-0.01 mmol, 5-10 mol \%) at room temperature. The resulting mixture was stirred for the specified time. The reaction mixture was concentrated, and the resulting residue was purified with silica gel column flash chromatography to yield the desired product.

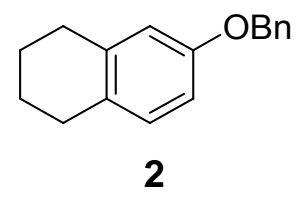

Compound 2 was isolated in $80 \%$ yield from acetate 1 following the general procedure B with 5 mol \% of $\mathrm{AuCl}$ and $2 \mathrm{~h}$ reaction time. ${ }^{1} \mathrm{H} \mathrm{NMR}\left(500 \mathrm{MHz}, \mathrm{CDCl}_{3}\right) \delta: 7.41(\mathrm{~d}, 2 \mathrm{H}, \mathrm{J}=7.5 \mathrm{~Hz}), 7.38$ $(\mathrm{t}, 2 \mathrm{H}, \mathrm{J}=6.0 \mathrm{~Hz}), 7.29(\mathrm{t}, 1 \mathrm{H}, \mathrm{J}=8.0 \mathrm{~Hz}), 6.96(\mathrm{~d}, 1 \mathrm{H}, \mathrm{J}=8.0 \mathrm{~Hz}), 6.73(\mathrm{~d}, 1 \mathrm{H}, \mathrm{J}=8.0 \mathrm{~Hz})$, $6.69(\mathrm{~s}, 1 \mathrm{H}), 5.02(\mathrm{~s}, 2 \mathrm{H}), 2.73-2.69(\mathrm{~m}, 4 \mathrm{H}), 1.78-1.76(\mathrm{~m}, 4 \mathrm{H}) ;{ }^{13} \mathrm{C} \mathrm{NMR}\left(125 \mathrm{MHz}, \mathrm{CDCl}_{3}\right) \delta$ : 156.6, 138.2, 137.4, 129.9, 129.6, 128.5, 127.7, 127.4, 114.7, 112.6, 69.9, 29.7, 28.6, 23.4, 23.1; IR (neat): 2929, 2253, 1607, 1496, 1455, 1384, 1260, 1231, 1049, 907, 731; MS (ES $)$ Calculated for $\left[\mathrm{C}_{17} \mathrm{H}_{18} \mathrm{NaO}\right]^{+} 261.1$; Found: 261.1.<smiles>Cc1cccc(OCc2ccccc2)c1</smiles>

$4 a$

Compound $\mathbf{4 a}$ was isolated in $67 \%$ yield from acetate $\mathbf{3 a}$ following the general procedure $\mathrm{B}$ with $5 \mathrm{~mol} \%$ of $\mathrm{AuCl}$ and $3 \mathrm{~h}$ reaction time. ${ }^{1} \mathrm{H}$ NMR $\left(500 \mathrm{MHz}, \mathrm{CDCl}_{3}\right) \delta: 7.44-7.30(\mathrm{~m} \mathrm{10H}), 7.17(\mathrm{t}$, $1 \mathrm{H}, \mathrm{J}=9.5 \mathrm{~Hz}), 6.81-6.77(\mathrm{~m}, 2 \mathrm{H}), 5.05(\mathrm{~s}, 2 \mathrm{H}), 2.33(\mathrm{~s}, 3 \mathrm{H})$.

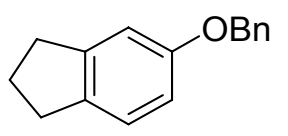

4b

Compound $\mathbf{4 b}$ was isolated in $99 \%$ yield from acetate $\mathbf{3 b}$ following the general procedure $\mathrm{B}$ with $5 \mathrm{~mol} \%$ of $\mathrm{AuCl}$ and $0.2 \mathrm{~h}$ reaction time. ${ }^{1} \mathrm{H}$ NMR $\left(500 \mathrm{MHz}, \mathrm{CDCl}_{3}\right)$ ס: 7.43-7.22 (m, 5H), 7.10 $(\mathrm{d}, 1 \mathrm{H}, \mathrm{J}=8.5 \mathrm{~Hz}), 6.86(\mathrm{~s}, 1 \mathrm{H}), 6.76(\mathrm{dd}, 1 \mathrm{H}, \mathrm{J}=8.5,2.5 \mathrm{~Hz}), 5.03(\mathrm{~s}, 2 \mathrm{H}), 2.87(\mathrm{t}, 2 \mathrm{H}, \mathrm{J}=8.0$ $\mathrm{Hz}$ ), $2.84(\mathrm{t}, 2 \mathrm{H}, \mathrm{J}=7.5 \mathrm{~Hz}), 2.09-2.03(\mathrm{~m}, 2 \mathrm{H}) ;{ }^{13} \mathrm{C} N M R\left(125 \mathrm{MHz}, \mathrm{CDCl}_{3}\right) \delta: 157.8,145.7$, $137.4,136.4,128.5,127.8,127.4,124.7,112.8,110.9,70.2$, 33.2, 31.9, 25.8; IR (neat): 2926, 2253, 1607, 1494, 1452, 1261, 1050, 907, 732; MS $\left(\mathrm{ES}^{+}\right)$Calculated for $\left[\mathrm{C}_{17} \mathrm{H}_{18} \mathrm{NaO}\right]^{+} 247.1$; Found: 247.1. 


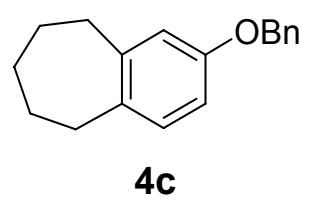

Compound $4 \mathrm{c}$ was isolated in $72 \%$ yield from acetate $3 \mathrm{c}$ following the general procedure $\mathrm{B}$ with $5 \mathrm{~mol} \%$ of $\mathrm{AuCl}$ and $3 \mathrm{~h}$ reaction time. ${ }^{1} \mathrm{H} \mathrm{NMR}\left(500 \mathrm{MHz}, \mathrm{CDCl}_{3}\right) \delta: 7.43-7.29(\mathrm{~m}, 5 \mathrm{H}), 7.00(\mathrm{~d}$, $1 \mathrm{H}, J=8.5 \mathrm{~Hz}), 6.77(\mathrm{~d}, 1 \mathrm{H}, \mathrm{J}=5.5 \mathrm{~Hz}), 6.68(\mathrm{dd}, 1 \mathrm{H}, \mathrm{J}=8.5,5.5 \mathrm{~Hz}), 5.02(\mathrm{~s}, 2 \mathrm{H}), 2.75-2.71$ $(\mathrm{m}, 4 \mathrm{H}), 1.83-1.78(\mathrm{~m}, 2 \mathrm{H}), 1.65-1.60(\mathrm{~m}, 4 \mathrm{H}) ;{ }^{13} \mathrm{C} \mathrm{NMR}\left(125 \mathrm{MHz}, \mathrm{CDCl}_{3}\right) \delta: 156.9,144.8$, 137.4, 136.0, 129.8, 128.5, 127.8, 127.4, 116.1, 111.1, 67.7, 36.9, 35.8, 32.7, 28.6, 28.4; IR (neat): 2925, 2253, 1607, 1495, 1267, 1049, 907, 732; MS (ES ${ }^{+}$Calculated for $\left[\mathrm{C}_{18} \mathrm{H}_{20} \mathrm{NaO}\right]^{+}$ 275.1; Found: 275.2 .

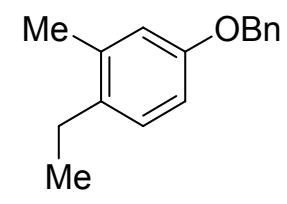

4d

Compound $\mathbf{4 d}$ was isolated in $83 \%$ yield from acetate $3 \mathbf{d}$ following the general procedure $B$ with $5 \mathrm{~mol} \%$ of $\mathrm{AuCl}$ and $3 \mathrm{~h}$ reaction time. ${ }^{1} \mathrm{H} \mathrm{NMR}\left(500 \mathrm{MHz}, \mathrm{CDCl}_{3}\right) \delta: 7.43-7.29(\mathrm{~m}, 5 \mathrm{H}), 7.05(\mathrm{~d}$, $1 \mathrm{H}, J=8.0 \mathrm{~Hz}), 6.79(\mathrm{~d}, 1 \mathrm{H}, \mathrm{J}=2.5 \mathrm{~Hz}), 6.76(\mathrm{dd}, 1 \mathrm{H}, \mathrm{J}=8.0,2.5 \mathrm{~Hz}), 5.03(\mathrm{~s}, 2 \mathrm{H}), 2.56(\mathrm{q}, 2 \mathrm{H}$,

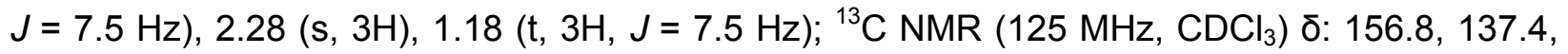
137.0, 134.9, 128.7, 128.5, 127.8, 127.4, 116.7, 111.9, 69.9, 25.4, 19.4, 14.7; IR (neat): 2926 , 1607, 1494, 1452, 1050, 906, 824, 731; MS $\left(\mathrm{ES}^{+}\right)$Calculated for $\left[\mathrm{C}_{16} \mathrm{H}_{18} \mathrm{NaO}\right]^{+} 249.1$; Found: 248.9.

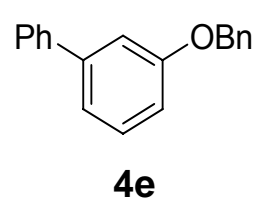

Compound $4 \mathrm{e}$ was isolated in $53 \%$ yield from acetate $3 \mathrm{e}$ following the general procedure $\mathrm{B}$ with $5 \mathrm{~mol} \%$ of $\mathrm{AuCl}$ and $3 \mathrm{~h}$ reaction time. ${ }^{1} \mathrm{H}$ NMR $\left(400 \mathrm{MHz}, \mathrm{CDCl}_{3}\right) \delta: 7.57(\mathrm{dd}, 2 \mathrm{H}, \mathrm{J}=0.8,6.4$ $\mathrm{Hz}), 7.46-7.21(\mathrm{~m}, 8 \mathrm{H}), 7.24-7.19(\mathrm{~m}, 2 \mathrm{H}), 6.96(\mathrm{dd}, 1 \mathrm{H}, \mathrm{J}=2.0,6.4 \mathrm{~Hz}), 5.12(\mathrm{~s}, 2 \mathrm{H}) ;{ }^{13} \mathrm{C} N M R$ $\left(125 \mathrm{MHz}, \mathrm{CDCl}_{3}\right)$ ס: 159.1, 142.8, 141.0, 137.0, 129.8, 128.7, 128.6, 127.9, 127.5, 127.4, 127.2, 119.9, 113.9, 113.5, 70.1; IR (neat): 2926, 2253, 1603, 1494, 1452, 1050, 907, 824, 732; MS $\left(\mathrm{ES}^{+}\right)$Calculated for $\left[\mathrm{C}_{19} \mathrm{H}_{16} \mathrm{NaO}\right]^{+}$283.1; Found: 283.0 . 


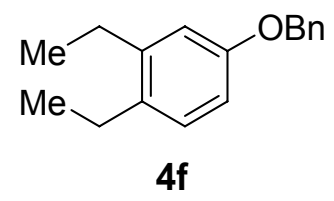

Compound $\mathbf{4 f}$ was isolated in $53 \%$ yield from acetate $\mathbf{3 f}$ following the general procedure $B$ with $10 \mathrm{~mol} \%$ of $\mathrm{AuCl}$ and $3 \mathrm{~h}$ reaction time. ${ }^{1} \mathrm{H}$ NMR $\left(400 \mathrm{MHz}, \mathrm{CDCl}_{3}\right) \delta: 7.44(\mathrm{~d}, 2 \mathrm{H}, \mathrm{J}=7.5 \mathrm{~Hz})$, $7.38(\mathrm{t}, 2 \mathrm{H}, \mathrm{J}=8.0 \mathrm{~Hz}), 7.31(\mathrm{t}, 1 \mathrm{H}, \mathrm{J}=7.5 \mathrm{~Hz}), 7.07(\mathrm{~d}, 1 \mathrm{H}, \mathrm{J}=8.5 \mathrm{~Hz}), 6.82(\mathrm{~d}, 1 \mathrm{H}, J=3.0$ $\mathrm{Hz}$ ), 6.77 (dd, $1 \mathrm{H}, \mathrm{J}=8.5,3.0 \mathrm{~Hz}$ ), $5.04(\mathrm{~s}, 2 \mathrm{H}), 2.62(\mathrm{q}, 2 \mathrm{H}, \mathrm{J}=7.5 \mathrm{~Hz}), 2.59$ (q, 2H, J = 7.5 $\mathrm{Hz}), 1.21(\mathrm{t}, 3 \mathrm{H}, J=7.5 \mathrm{~Hz}), 1.19(\mathrm{t}, 3 \mathrm{H}, \mathrm{J}=7.5 \mathrm{~Hz}) ;{ }^{13} \mathrm{C} \mathrm{NMR}\left(125 \mathrm{MHz}, \mathrm{CDCl}_{3}\right) \delta: 157.0$, 143.0, 137.3, 134.2, 129.1, 128.5, 127.8, 127.5, 115.0, 111.6, 69.9, 25.6, 24.7, 15.5, 15.1; IR (neat): 2928, 2253, 1607, 1494, 1454, 1262, 1050, 907, 824, 732; MS (ES ${ }^{+}$) Calculated for $\left[\mathrm{C}_{17} \mathrm{H}_{20} \mathrm{NaO}\right]^{+}$263.1; Found: 263.0 .

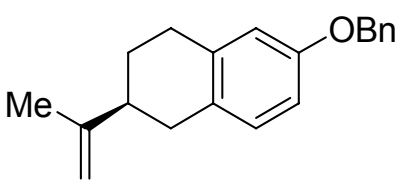

49

Compound $\mathbf{4 g}$ was isolated in $65 \%$ yield from acetate $\mathbf{3 g}$ following the general procedure $B$ with $10 \mathrm{~mol} \%$ of AuCl and $10 \mathrm{~h}$ reaction time. ${ }^{1} \mathrm{H} \mathrm{NMR}\left(400 \mathrm{MHz}, \mathrm{CDCl}_{3}\right) \delta: 7.44(\mathrm{~d}, 2 \mathrm{H}, \mathrm{J}=8.5 \mathrm{~Hz})$, $7.38(\mathrm{t}, 2 \mathrm{H}, \mathrm{J}=7.5 \mathrm{~Hz}), 7.33-7.30(\mathrm{~m}, 1 \mathrm{H}), 6.99(\mathrm{~d}, 1 \mathrm{H}, \mathrm{J}=8.0 \mathrm{~Hz}), 6.76(\mathrm{dd}, 1 \mathrm{H}, J=1.0,8.0$ $\mathrm{Hz}), 6.72(\mathrm{~d}, 1 \mathrm{H}, \mathrm{J}=1.0 \mathrm{~Hz}), 5.03(\mathrm{~s}, 2 \mathrm{H}), 4.78-4.75(\mathrm{~m}, 2 \mathrm{H}), 2.84-2.79(\mathrm{~m}, 3 \mathrm{H}), 2.63(\mathrm{dd}, 1 \mathrm{H}$, $\mathrm{J}=11.0,16.0 \mathrm{~Hz}), 2.35-2.29(\mathrm{~m}, 1 \mathrm{H}), 1.99-1.94(\mathrm{~m}, 1 \mathrm{H}), 1.80(\mathrm{~s}, 3 \mathrm{H}), 1.66-1.58(\mathrm{~m}, 1 \mathrm{H}) ;{ }^{13} \mathrm{C}$ $\operatorname{NMR}\left(125 \mathrm{MHz}, \mathrm{CDCl}_{3}\right) \delta: 156.8,149.6,137.5,137.3,129.9,129.1,128.5,127.8,127.4,114.5$, 112.7, 108.9, 69.9, 41.9, 34.1, 29.8, 28.1, 20.8; IR (neat): 2926, 2253, 1607, 1494, 1452, 1262, 1050, 907, 824, 732; MS (ES $\left.{ }^{+}\right)$Calculated for $\left[\mathrm{C}_{20} \mathrm{H}_{22} \mathrm{NaO}\right]^{+} 301.2$; Found: 300.9.

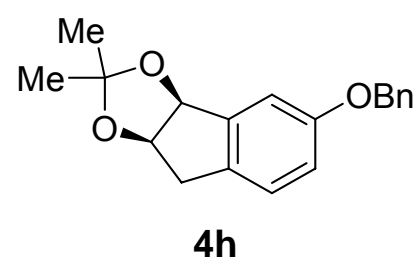

Compound $4 \mathrm{~h}$ was isolated in $60 \%$ yield from acetate $3 \mathrm{~h}$ following the general procedure $\mathrm{B}$ with $10 \mathrm{~mol} \%$ of $\mathrm{AuCl}$ and $6 \mathrm{~h}$ reaction time. ${ }^{1} \mathrm{H}$ NMR $\left(400 \mathrm{MHz}, \mathrm{CDCl}_{3}\right)$ ס: 7.44-7.29 (m, 5H), 7.12 $(\mathrm{d}, 1 \mathrm{H}, \mathrm{J}=8.0 \mathrm{~Hz}), 7.03(\mathrm{~d}, 1 \mathrm{H}, \mathrm{J}=2.4 \mathrm{~Hz}), 6.93(\mathrm{dd}, 1 \mathrm{H}, \mathrm{J}=8.0,2.4 \mathrm{~Hz}), 5.49(\mathrm{~d}, 1 \mathrm{H}, \mathrm{J}=5.6$ $\mathrm{Hz}), 5.07(\mathrm{~d}, 1 \mathrm{H}, \mathrm{J}=11.6 \mathrm{~Hz}), 5.03(\mathrm{~d}, 1 \mathrm{H}, \mathrm{J}=11.6 \mathrm{~Hz}), 4.97(\mathrm{dt}, 1 \mathrm{H}, J=2.0,5.6 \mathrm{~Hz}), 3.17-$ $3.03(\mathrm{~m}, 2 \mathrm{H}), 1.42(\mathrm{~s}, 3 \mathrm{H}), 1.24(\mathrm{~s}, 3 \mathrm{H}) ;{ }^{13} \mathrm{C}$ NMR $\left(125 \mathrm{MHz}, \mathrm{CDCl}_{3}\right) \delta: 158.4,142.7,136.7$, 132.9, 128.5, 127.9, 127.5, 126.1, 116.8, 110.9, 110.8, 84.1, 80.1, 70.2, 37.1, 27.5, 25.9; IR 
(neat): 2927, 2251, 1609, 1494, 1453, 1383, 1251, 1154, 1050, 907, 824, 732; MS (ES $\left.{ }^{+}\right)$ Calculated for $\left[\mathrm{C}_{19} \mathrm{H}_{20} \mathrm{NaO}_{3}\right]^{+}$319.1; Found: 318.9.

\section{General procedures C: AuCl-catalyzed synthesis of benzyl-protected substituted phenols} from enone starting materials.

To a solution of 1-benzyloxy-1,2-propadiene (175 mg, $1.2 \mathrm{mmol}, 1.2$ eq.) in $10 \mathrm{~mL}$ of THF cooled in a dry ice-acetone bath was added $n$-BuLi $(0.75 \mathrm{~mL}, 1.2 \mathrm{mmol}, 1.2$ eq.) dropwise under $\mathrm{N}_{2}$. The mixture was stirred at the same temperature for $1 \mathrm{~h}$ before the addition of TMEDA (0.2 $\mathrm{mL}, 1.2 \mathrm{mmol}, 1.2$ eq.). After stirring for $15 \mathrm{~min}$, an enone (1 mmol, 1 eq.) was added dropwise, and the resulting mixture was stirred for another $2 \mathrm{~h}$. The thus-formed lithium alkoxide was treated with $\mathrm{BzCl}(0.16 \mathrm{~mL}, 1.4 \mathrm{mmol}, 1.4$ eq.). The reaction was warmed to room temperature and quenched with water. The resulting mixture was extracted with ether $(3 \times 50 \mathrm{~mL})$, and the combined organic layers were washed with $\mathrm{H}_{2} \mathrm{O}(50 \mathrm{~mL})$ and brine $(20 \mathrm{~mL})$. The solvent was evaporated under vacuum, and the crude benzoates were used directly for the AuCl-catalyzed reaction (the purity of benzoates was calculated by dividing the theoretical weight with the weight of the crude residue).

To a solution of the crude benzoates prepared above $\left(0.1 \mathrm{mmol}, 1\right.$ eq.) in $2 \mathrm{~mL}$ of $\mathrm{CH}_{2} \mathrm{Cl}_{2}$ was added $\mathrm{AuCl}(0.005-0.01 \mathrm{mmol}, 5 \%)$ at room temperature. The resulting mixture was stirred for $3 \mathrm{~h}$. The solvent was evaporated, and the residue was treated with $\mathrm{KOH}$ (2 eq.) in refluxing Methanol/ $/ \mathrm{H}_{2} \mathrm{O}(1 / 1)$ for $2 \mathrm{~h}$. It was then cooled and extracted with ethyl ether $(3 \times 10 \mathrm{~mL})$. The combined organic layers were dried $\left(\mathrm{MgSO}_{4}\right)$, filtered, and concentrated under vacuum. The residue was purified with silica gel flash column chromatography to yield the desired benzylprotected substituted phenol.

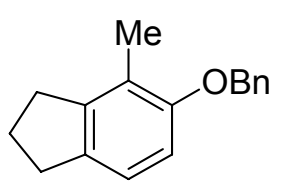

$6 a$

Compound $6 \mathrm{a}$ was isolated in $70 \%$ overall yield from cyclopent-1-enylethanone following the general procedure C. ${ }^{1} \mathrm{H}$ NMR $\left(500 \mathrm{MHz}, \mathrm{CDCl}_{3}\right) \delta: 7.44(\mathrm{~d}, 2 \mathrm{H}, J=7.5 \mathrm{~Hz}), 7.39-7.29(\mathrm{~m}, 3 \mathrm{H})$, $6.98(\mathrm{~d}, 1 \mathrm{H}, \mathrm{J}=8.5 \mathrm{~Hz}), 6.71(\mathrm{~d}, 1 \mathrm{H}, \mathrm{J}=8.5 \mathrm{~Hz}), 5.04(\mathrm{~s}, 2 \mathrm{H}), 2.87(\mathrm{t}, 2 \mathrm{H}, \mathrm{J}=7.5 \mathrm{~Hz}), 2.84(\mathrm{t}$, $2 \mathrm{H}, J=7.5 \mathrm{~Hz}), 2.20(\mathrm{~s}, 3 \mathrm{H}), 2.07(\mathrm{p}, 2 \mathrm{H}, \mathrm{J}=7.5 \mathrm{~Hz}),{ }^{13} \mathrm{C} \mathrm{NMR}\left(125 \mathrm{MHz}, \mathrm{CDCl}_{3}\right) \delta: 155.4$, 144.8, 137.8, 136.2, 128.4, 128.39, 127.8, 127.6, 127.1, 122.9, 110.1, 70.5, 32.5, 31.8, 25.4, 12.6; IR (neat): 2925, 2253, 1607, 1494, 1452, 1252, 1050, 908, 732; MS (ES ${ }^{+}$) Calculated for $\left[\mathrm{C}_{17} \mathrm{H}_{18} \mathrm{NaO}\right]^{+}$261.1; Found: 261.1. 


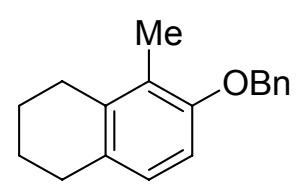

$6 b$

Compound $\mathbf{6 b}$ was isolated in $66 \%$ overall yield from cyclohex-1-enylethanone following the general procedure C. ${ }^{1} \mathrm{H}$ NMR $\left(500 \mathrm{MHz}, \mathrm{CDCl}_{3}\right) \delta: 7.44(\mathrm{~d}, 2 \mathrm{H}, \mathrm{J}=6.0 \mathrm{~Hz}), 7.38(\mathrm{t}, 2 \mathrm{H}, \mathrm{J}=8.0$ $\mathrm{Hz}), 7.30(\mathrm{t}, 1 \mathrm{H}, \mathrm{J}=7.5 \mathrm{~Hz}$ ), $6.87(\mathrm{~d}, 1 \mathrm{H}, \mathrm{J}=8.0 \mathrm{~Hz}), 6.73(\mathrm{~d}, 1 \mathrm{H}, \mathrm{J}=8.0 \mathrm{~Hz}), 5.04(\mathrm{~s}, 2 \mathrm{H}), 2.72$ (t, $2 \mathrm{H}, J=6.5 \mathrm{~Hz}), 2.65(\mathrm{t}, 2 \mathrm{H}, \mathrm{J}=6.5 \mathrm{~Hz}), 2.17(\mathrm{~s}, 3 \mathrm{H}), 1.84-1.81(\mathrm{~m}, 2 \mathrm{H}), 1.76-1.72(\mathrm{~m}, 2 \mathrm{H})$; ${ }^{13} \mathrm{C} \mathrm{NMR}\left(125 \mathrm{MHz}, \mathrm{CDCl}_{3}\right) \delta: 154.5,137.9,136.7,129.8,128.4,127.6,127.1,126.7,125.2$, 109.7, 70.5, 29.6, 27.2, 23.4, 22.9, 11.2; IR (neat): 2928, 2253, 1603, 1494, 1452, 1262, 1050 , 907, 732; MS (ES $\left.{ }^{+}\right)$Calculated for $\left[\mathrm{C}_{18} \mathrm{H}_{20} \mathrm{NaO}^{+} 275.1\right.$; Found: 274.9 .

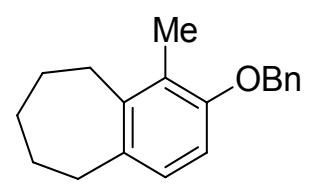

6c

Compound $6 \mathrm{c}$ was isolated in $53 \%$ overall yield from cyclohept-1-enylethanone following the general procedure C. ${ }^{1} \mathrm{H}$ NMR $\left(500 \mathrm{MHz}, \mathrm{CDCl}_{3}\right) \delta: 7.45(\mathrm{dd}, 2 \mathrm{H}, \mathrm{J}=1.5,8.0 \mathrm{~Hz}), 7.39-7.29(\mathrm{~m}$, $3 \mathrm{H}), 6.89(\mathrm{~d}, 1 \mathrm{H}, \mathrm{J}=8.5 \mathrm{~Hz}), 6.65(\mathrm{~d}, 1 \mathrm{H}, \mathrm{J}=8.5 \mathrm{~Hz}), 5.02(\mathrm{~s}, 2 \mathrm{H}), 2.83-2.81(\mathrm{~m}, 2 \mathrm{H}), 2.76-$ $2.73(\mathrm{~m}, 2 \mathrm{H}), 2.27(\mathrm{~s}, 3 \mathrm{H}), 1.83-1.78(\mathrm{~m}, 2 \mathrm{H}), 1.63-1.58(\mathrm{~m}, 4 \mathrm{H}){ }^{13} \mathrm{C} \mathrm{NMR}\left(125 \mathrm{MHz}, \mathrm{CDCl}_{3}\right) \delta$ : 155.1, 143.2, 137.8, 136.4, 128.4, 128.39, 127.8, 127.6, 127.2, 124.1, 108.7, 70.2, 35.9, 32.4, 30.0, 28.7, 27.2, 11.9; IR (neat): 2925, 2253, 1607, 1494, 1452, 1262, 1050, 908, 732; MS (ES $\left.{ }^{+}\right)$ Calculated for $\left[\mathrm{C}_{19} \mathrm{H}_{22} \mathrm{NaO}\right]^{+}$289.1; Found: 289.0.

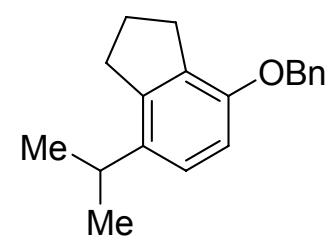

$6 d$

Compound $6 \mathbf{d}$ was isolated in $64 \%$ overall yield from 2-isobutylidenecyclopentanone following the general procedure C. ${ }^{1} \mathrm{H} \mathrm{NMR}\left(500 \mathrm{MHz}, \mathrm{CDCl}_{3}\right)$ ס: $7.44(\mathrm{dd}, 2 \mathrm{H}, \mathrm{J}=1.5,8.5 \mathrm{~Hz}), 7.39-7.29$ $(\mathrm{m}, 3 \mathrm{H}), 7.00(\mathrm{~d}, 1 \mathrm{H}, \mathrm{J}=8.5 \mathrm{~Hz}), 6.71(\mathrm{~d}, 1 \mathrm{H}, \mathrm{J}=8.5 \mathrm{~Hz}), 5.07(\mathrm{~s}, 2 \mathrm{H}), 2.96-2.91(\mathrm{~m}, 5 \mathrm{H})$, 2.11-2.05 (m, 2H), $1.21(\mathrm{~d}, 6 \mathrm{H}, \mathrm{J}=6.5 \mathrm{~Hz}) ;{ }^{13} \mathrm{C}$ NMR $\left(125 \mathrm{MHz}, \mathrm{CDCl}_{3}\right)$ ठ: 153.3, 143.8, 137.8, $137.1,131.9,128.4,127.6,127.1,123.4,109.6,69.7,31.6,30.4,29.8,24.6,23.2$; IR (neat): 
2926, 2253, 1607, 1494, 1452, 1264, 1050, 907, 732; $M S\left(E S^{+}\right)$Calculated for $\left[\mathrm{C}_{19} \mathrm{H}_{22} \mathrm{NaO}^{+}\right.$ 289.2; Found: 289.0.

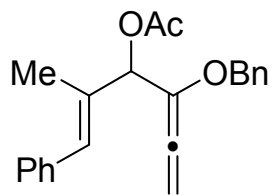

7

Compound 7 was synthesized according to the general procedure $A$ using $\alpha$ methylcinnamaldehyde. Yield: $69 \%$; ${ }^{1} \mathrm{H}$ NMR $\left(400 \mathrm{MHz}, \mathrm{CDCl}_{3}\right)$ ס: 7.38-7.21 (m, 10H), $6.61(\mathrm{~s}$, 1H), $5.85(\mathrm{~s}, 1 \mathrm{H}), 5.55(\mathrm{~d}, 2 \mathrm{H}, \mathrm{J}=2.5 \mathrm{~Hz}), 4.72-4.64(\mathrm{~m}, 2 \mathrm{H}), 2.13(\mathrm{~s}, 3 \mathrm{H}), 1.86(\mathrm{~d}, 3 \mathrm{H}, \mathrm{J}=1.5$ $\mathrm{Hz}) ;{ }^{13} \mathrm{C}$ NMR $\left(125 \mathrm{MHz}, \mathrm{CDCl}_{3}\right) \delta: 198.8,169.8,137.4,137.2,133.4,130.9,129.1,128.6$, 128.3, 128.1, 127.9, 127.7, 127.4, 126.7, 92.4, 70.7, 53.4, 21.2, 14.6; IR (neat): 2926, 2253, $\left.1734,1607,1494,1451,1384,1239,1050,907,734 ; \mathrm{MS}_{(E S}{ }^{+}\right)$Calculated for $\left[\mathrm{C}_{22} \mathrm{H}_{22} \mathrm{NaO}_{3}\right]^{+}$ 357.1; Found: 356.9.

\section{Mechanism studies:}

To a solution of 7 ( $50 \mathrm{mg}, 0.145 \mathrm{mmol}, 1$ eq.) in $3 \mathrm{~mL}$ of dichloromethane was added $\mathrm{AuCl}$ ( 3.4 $\mathrm{mg}, 0.0145 \mathrm{mmol}, 0.1$ eq.) in one portion. The mixture was stirred at rt for $30 \mathrm{~min}$ and TLC showed complete consumption of $\mathbf{7}$ and formation of intermediate $\mathbf{8}$ and phenol product $\mathbf{9}$. The column purification was done on basified silica gel column.

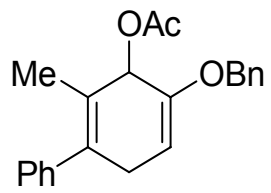

8

Yield: 44\%. ${ }^{1} \mathrm{H}$ NMR $\left(400 \mathrm{MHz}, \mathrm{CDCl}_{3}\right) \delta: 7.45-7.15(\mathrm{~m}, 10 \mathrm{H}), 5.51$ (dd, $1 \mathrm{H}, \mathrm{J}=2.8,6.8 \mathrm{~Hz}$ ), $5.35(\mathrm{~s}, 2 \mathrm{H}), 4.90(\mathrm{~s}, 2 \mathrm{H}), 3.03-2.96(\mathrm{~m}, 1 \mathrm{H}), 2.78(\mathrm{ddd}, 1 \mathrm{H}, \mathrm{J}=18.4,2.4,0.8 \mathrm{~Hz}), 2.06(\mathrm{~s}, 3 \mathrm{H})$, $1.86(\mathrm{dd}, 3 \mathrm{H}, \mathrm{J}=1.2,2.8 \mathrm{~Hz}){ }^{13} \mathrm{C}$ NMR $\left(125 \mathrm{MHz}, \mathrm{CDCl}_{3}\right)$ ס: 170.6, 152.6, 141.7, 136.4, 128.5, 128.4, 128.0, 127.9, 127.3, 126.2, 125.4, 123.6, 104.2, 69.6, 68.7, 37.0, 21.4, 19.8; IR (neat): 3390, 2926, 2253, 1730, 1654, 1601, 1455, 1371, 1246, 1188, 1104, 1016, 981, 907, 733; MS $\left(\mathrm{ES}^{+}\right)$Calculated for $\left[\mathrm{C}_{22} \mathrm{H}_{22} \mathrm{NaO}\right]^{+}$357.2; Found: 356.9. 


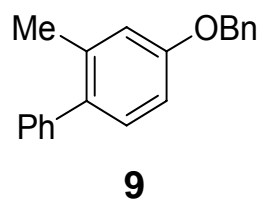

Yield: 40\%; ${ }^{1} \mathrm{H}$ NMR (500MHz, $\left.\mathrm{CDCl}_{3}\right)$ ס: 7.46-7.30 (m 10H), 7.15 (d, $\left.1 \mathrm{H}, \mathrm{J}=8.5 \mathrm{~Hz}\right), 6.91(\mathrm{~d}$, $1 \mathrm{H}, \mathrm{J}=2.5 \mathrm{~Hz}$ ), $6.86(\mathrm{dd}, 1 \mathrm{H}, \mathrm{J}=8.5,2.5 \mathrm{~Hz}), 5.09(\mathrm{~s}, 2 \mathrm{H}), 2.26(\mathrm{~s}, 3 \mathrm{H}) ;{ }^{13} \mathrm{C} \mathrm{NMR}(125 \mathrm{MHz}$, $\left.\mathrm{CDCl}_{3}\right)$ ס: 157.9, 141.5, 137.1, 136.8, 134.9, 130.8, 129.4, 128.6, 128.0, 127.9, 127.5, 126.5, 116.6, 111.8, 69.9, 20.8; IR (neat): 2926, 2253, 1607, 1494, 1453, 1235, 1050, 908, 824, 731; MS $\left(\mathrm{ES}^{+}\right)$Calculated for $\left[\mathrm{C}_{20} \mathrm{H}_{18} \mathrm{NaO}\right]^{+}$297.1; Found: 297.1. 


$$
=
$$



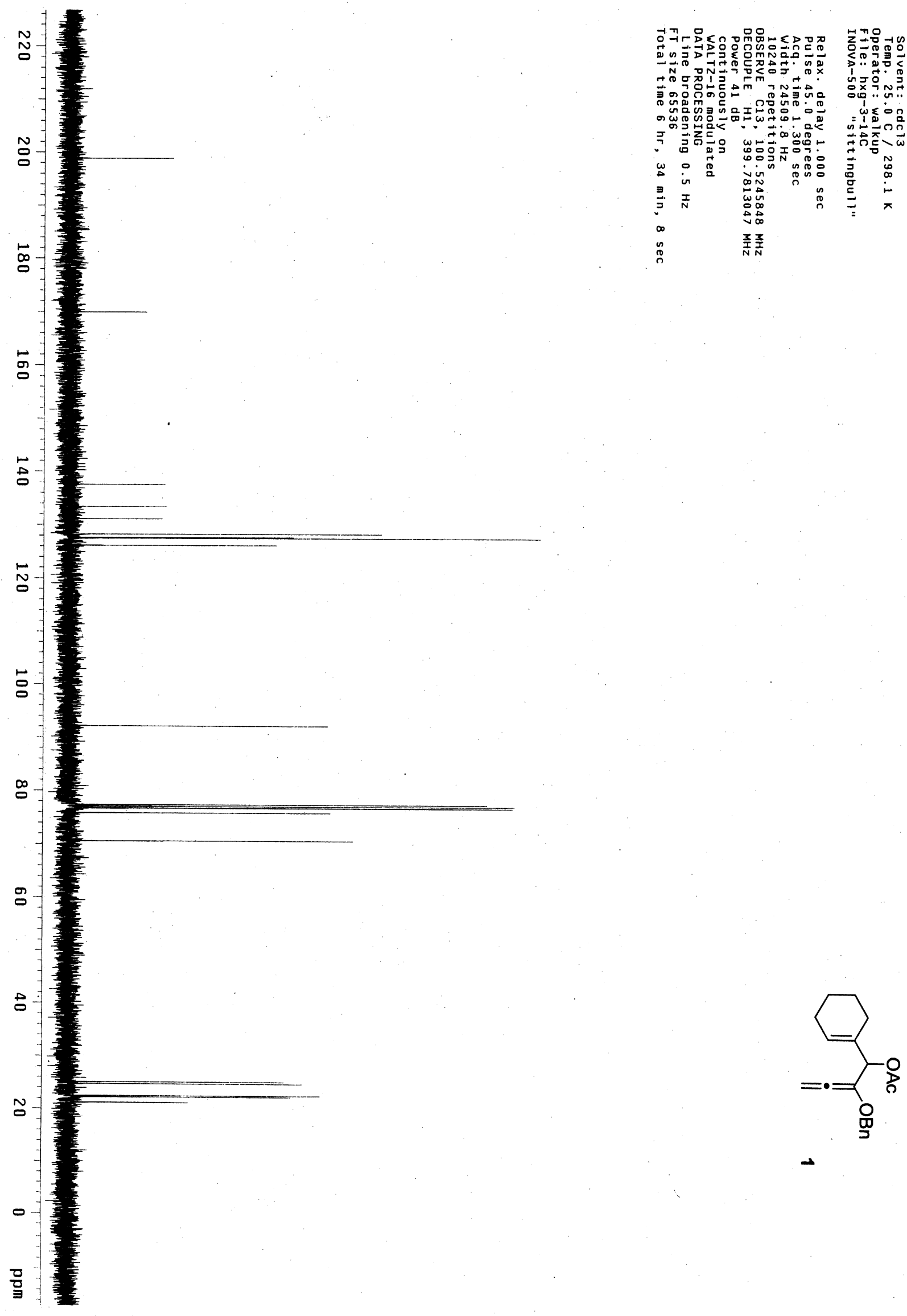

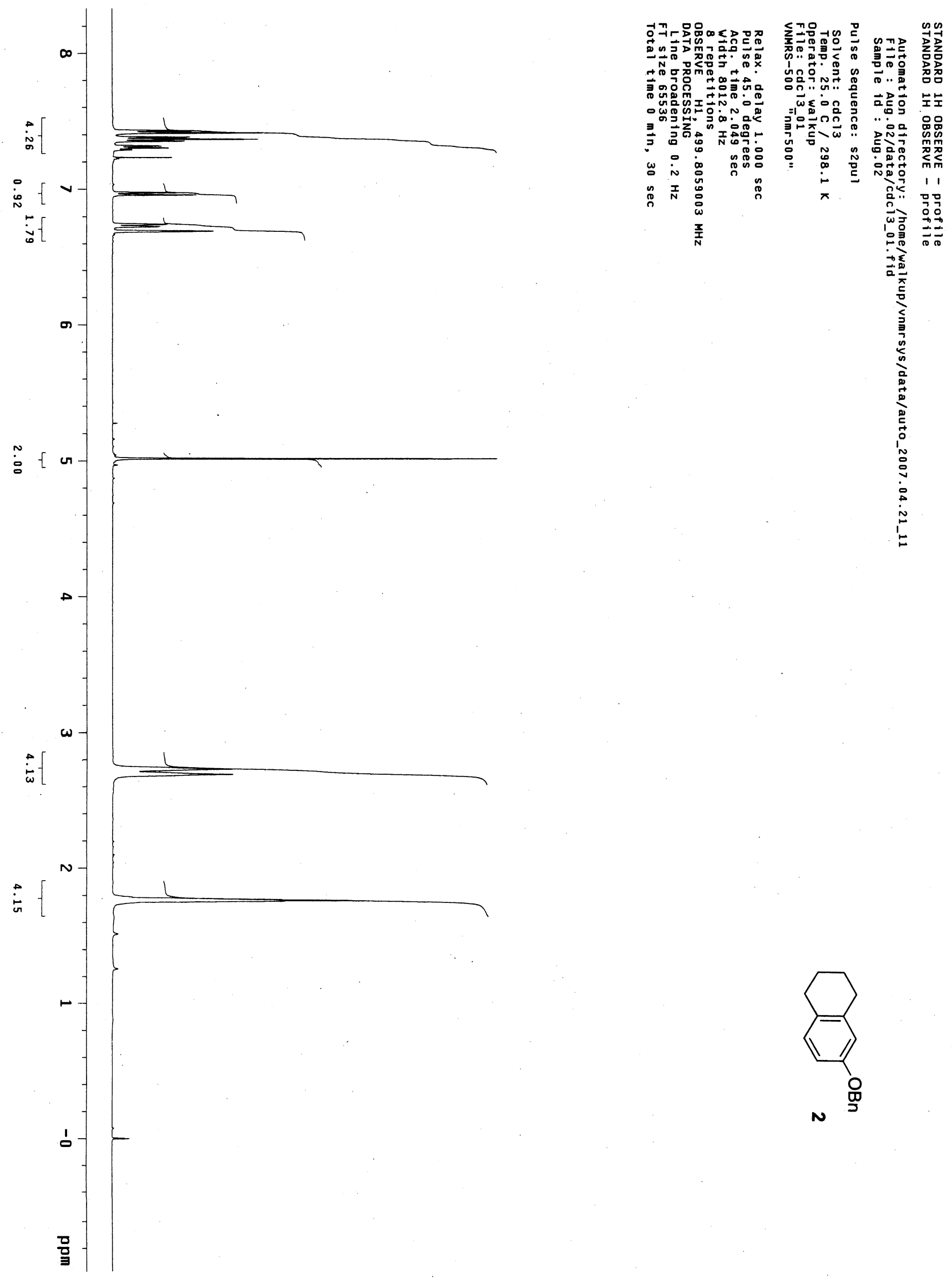


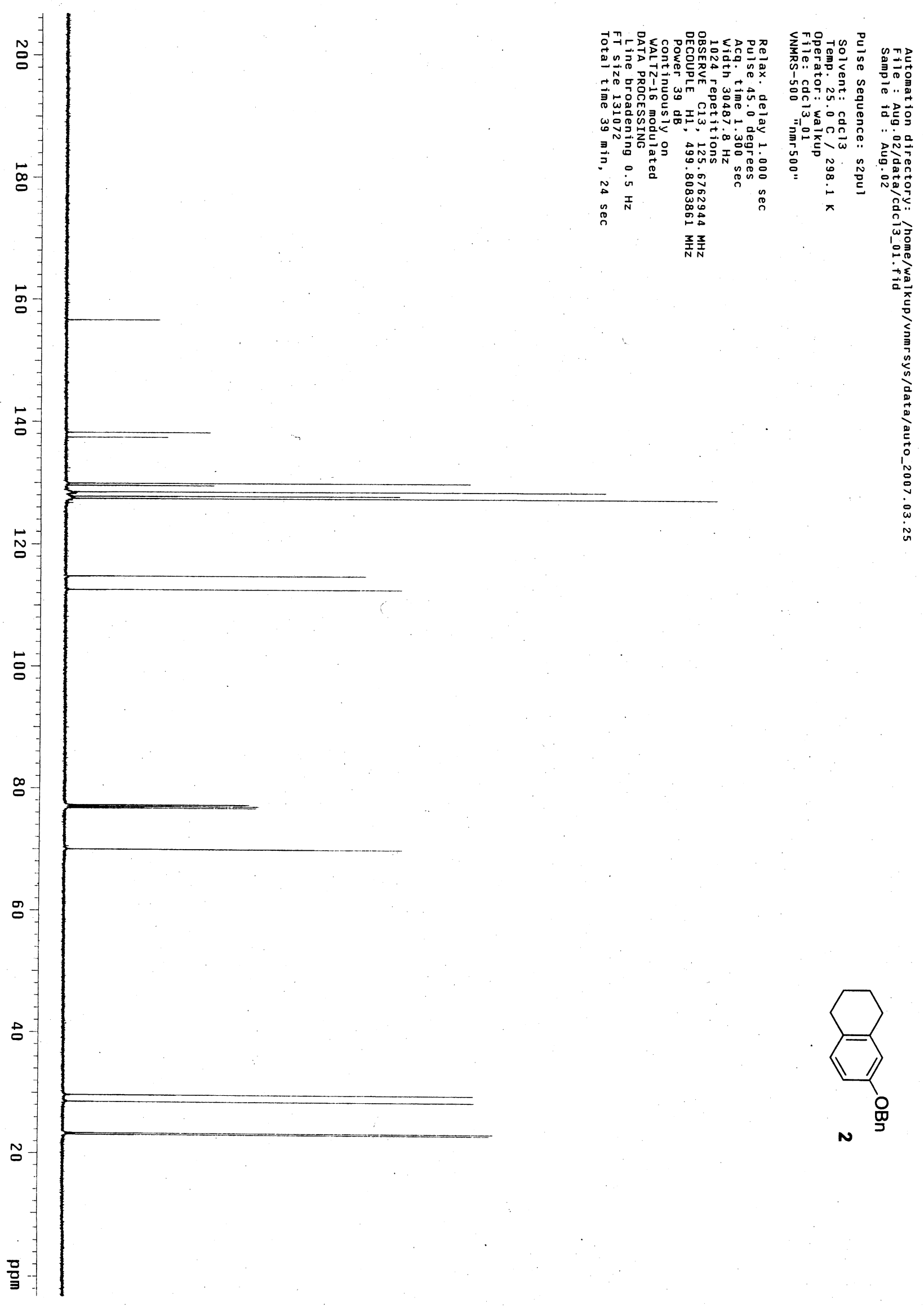




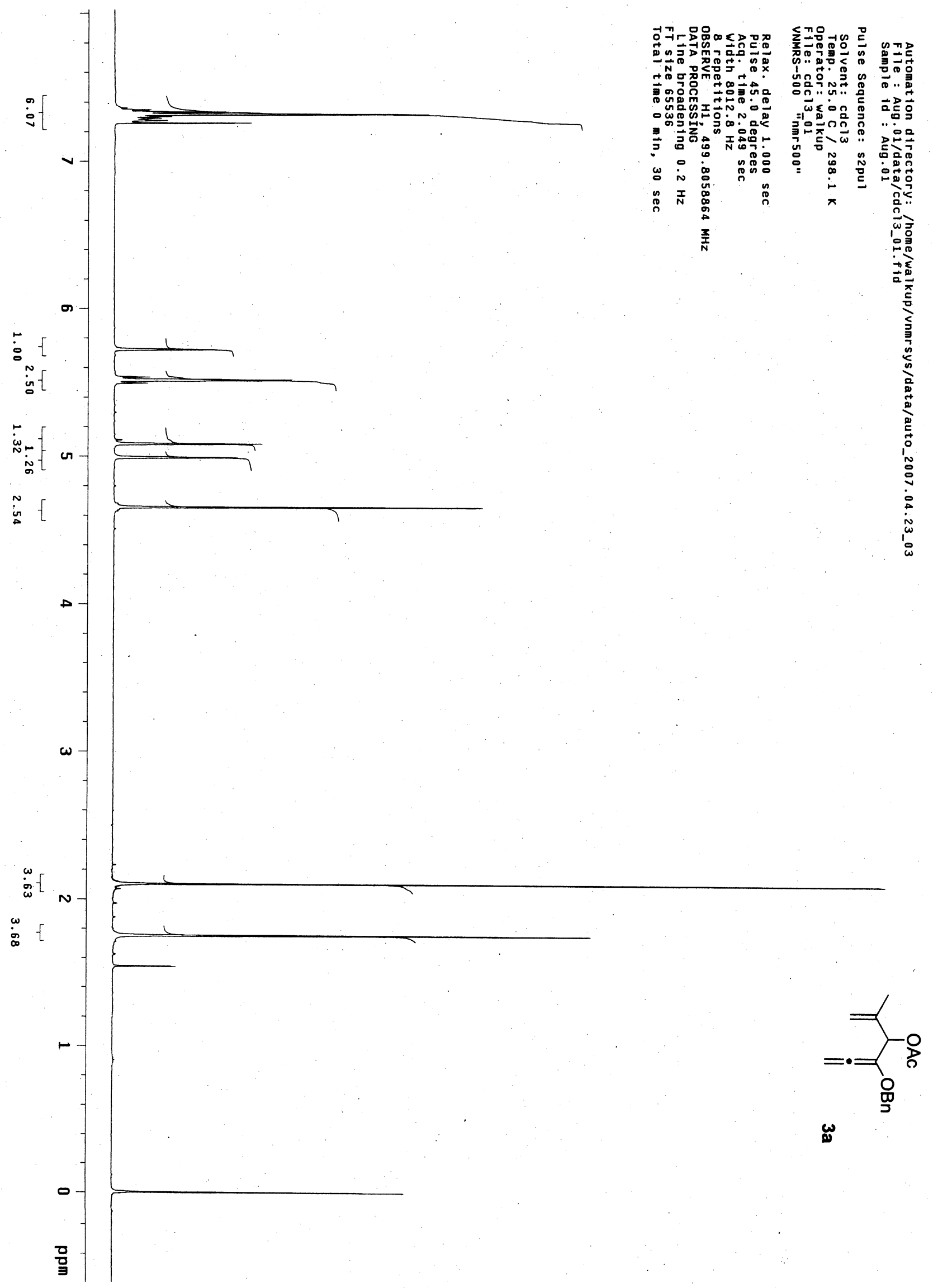




$$
\text { |"7 }
$$




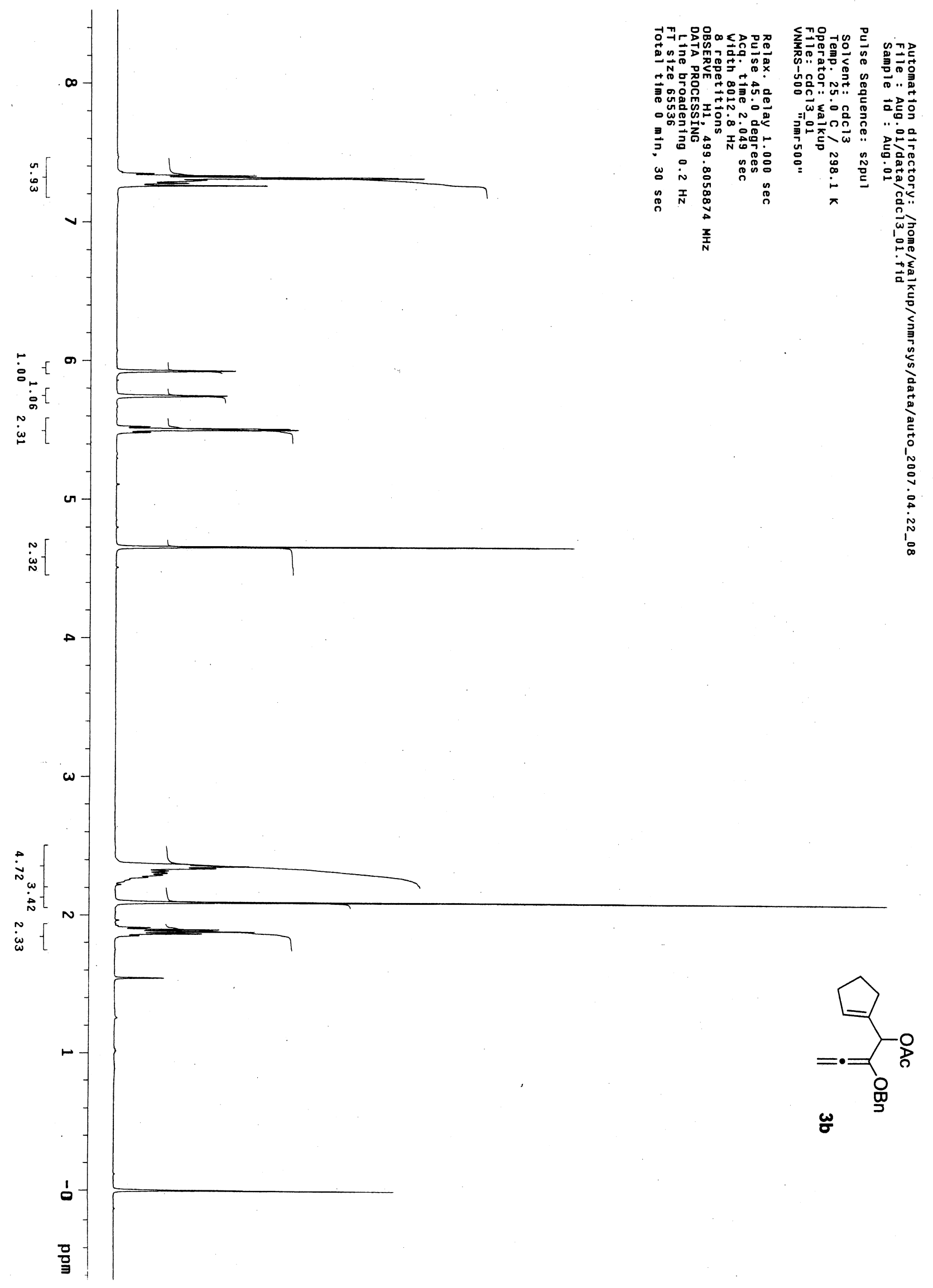




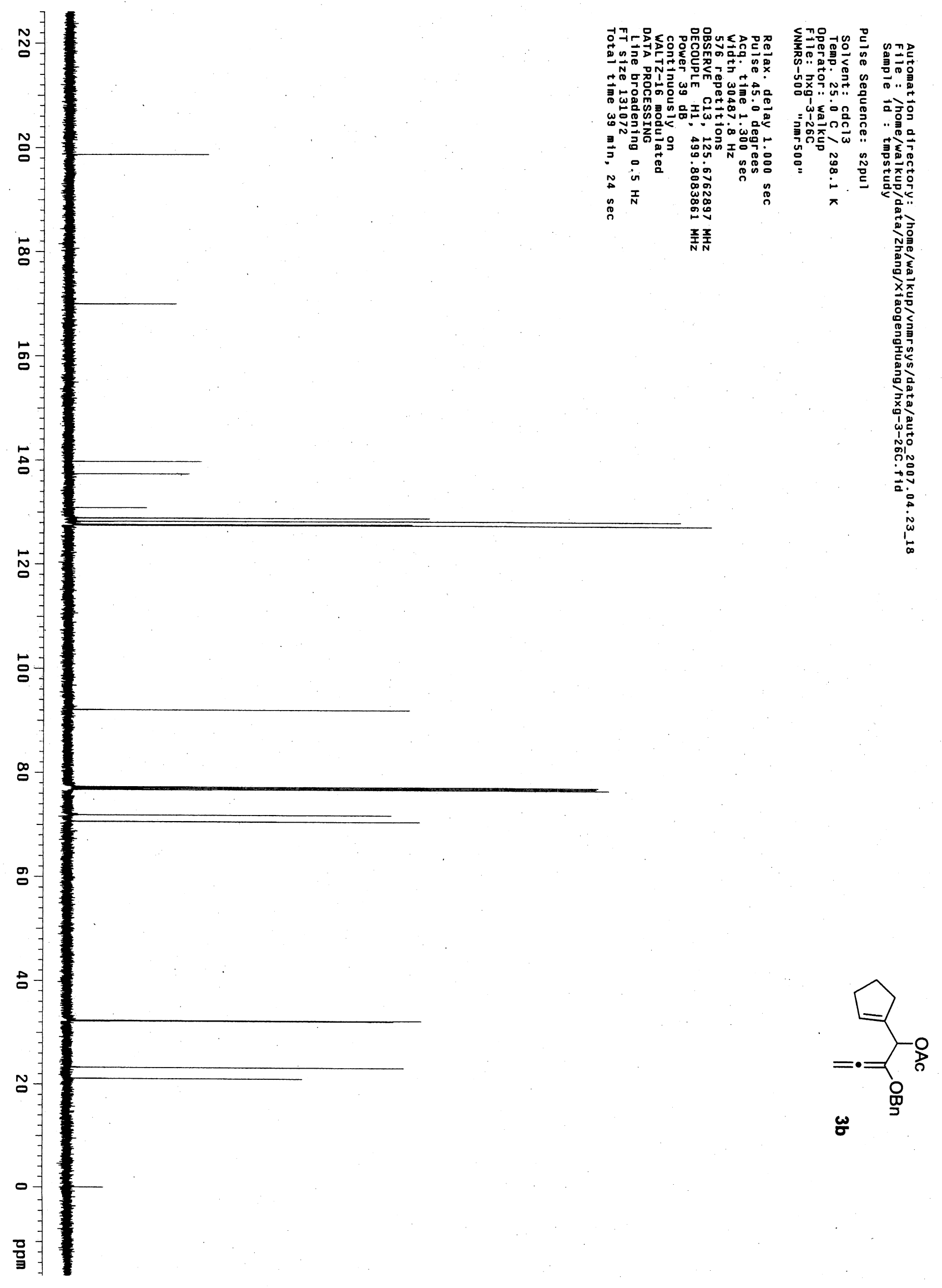



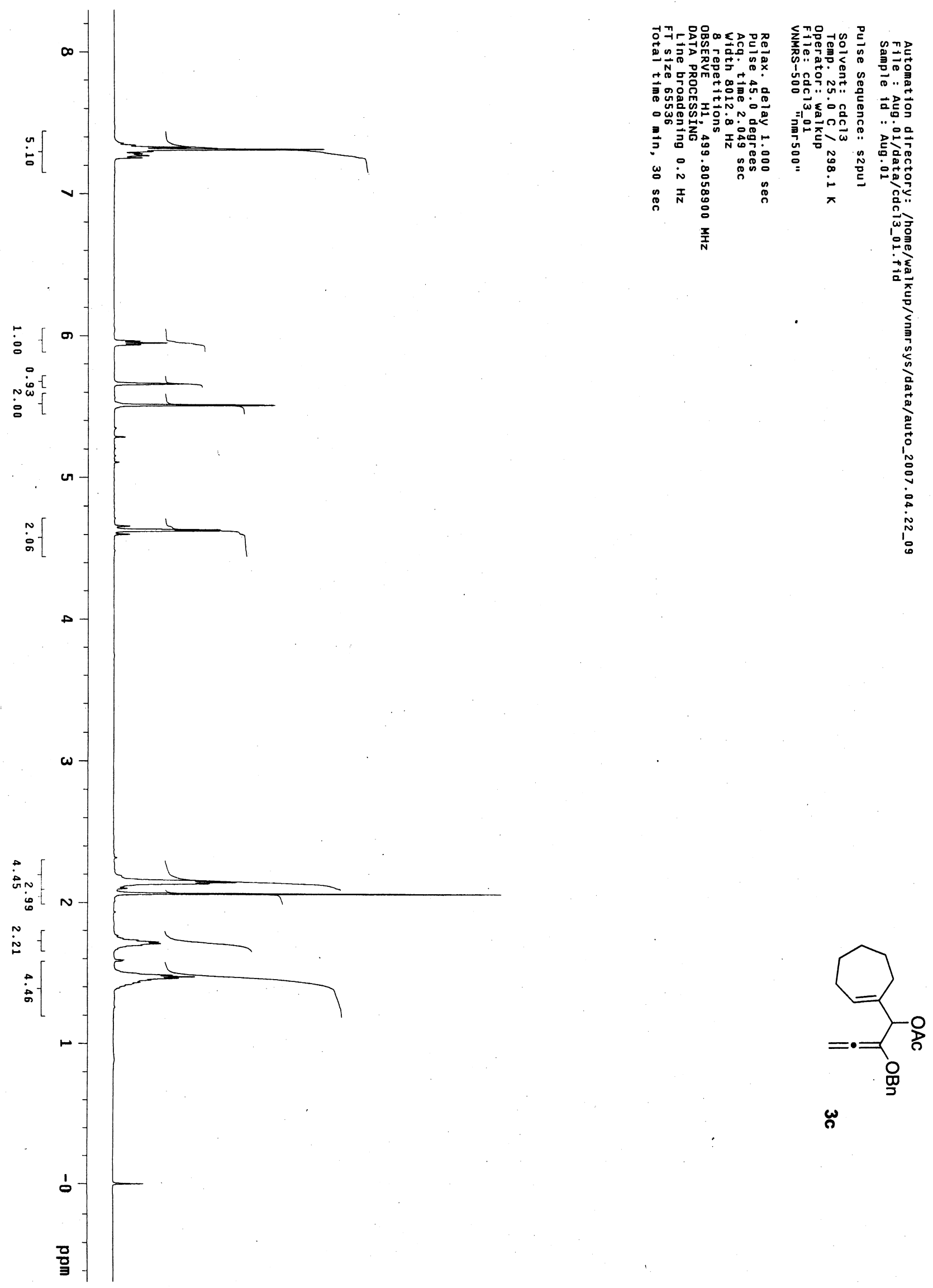

ณ 


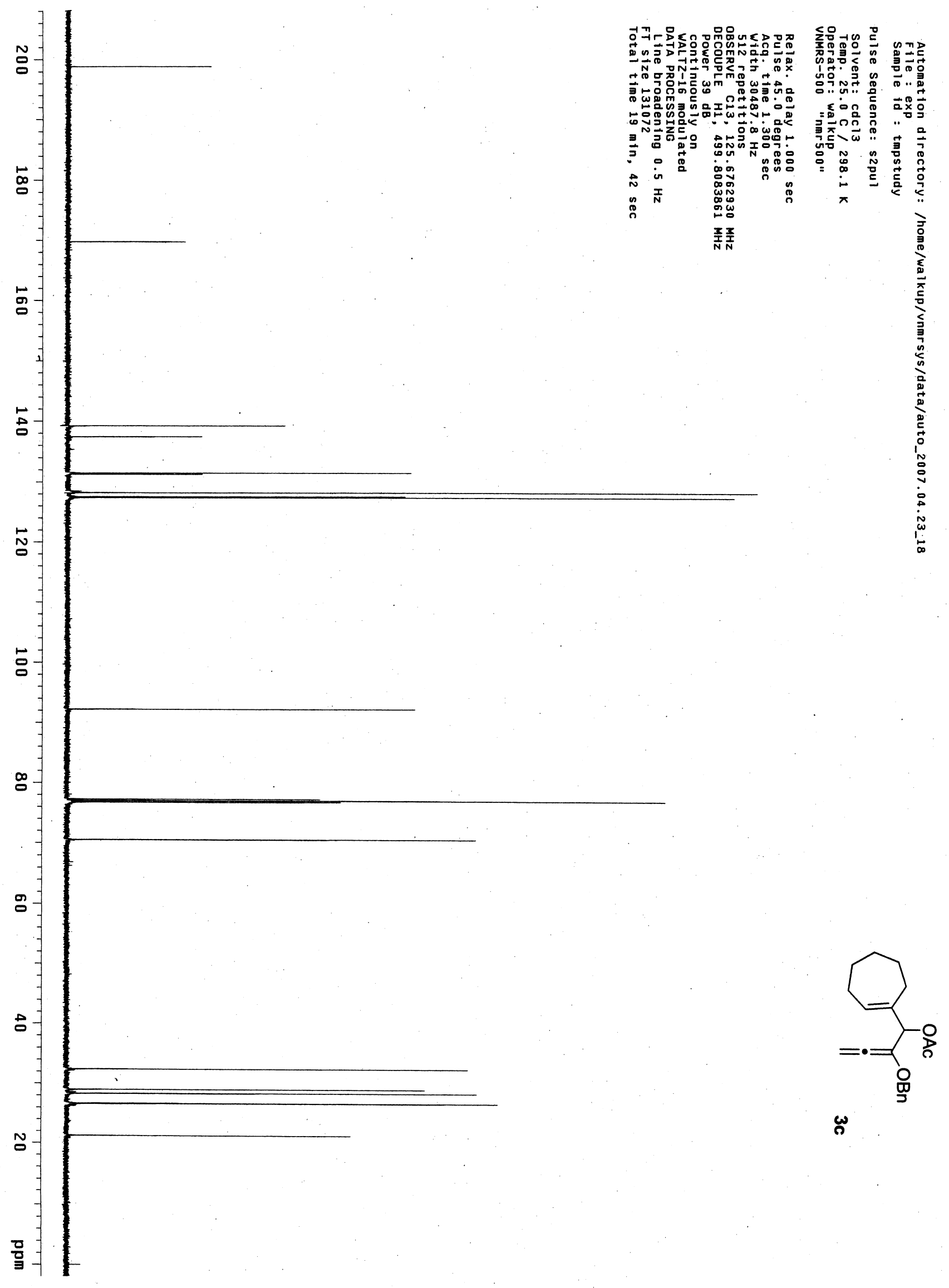




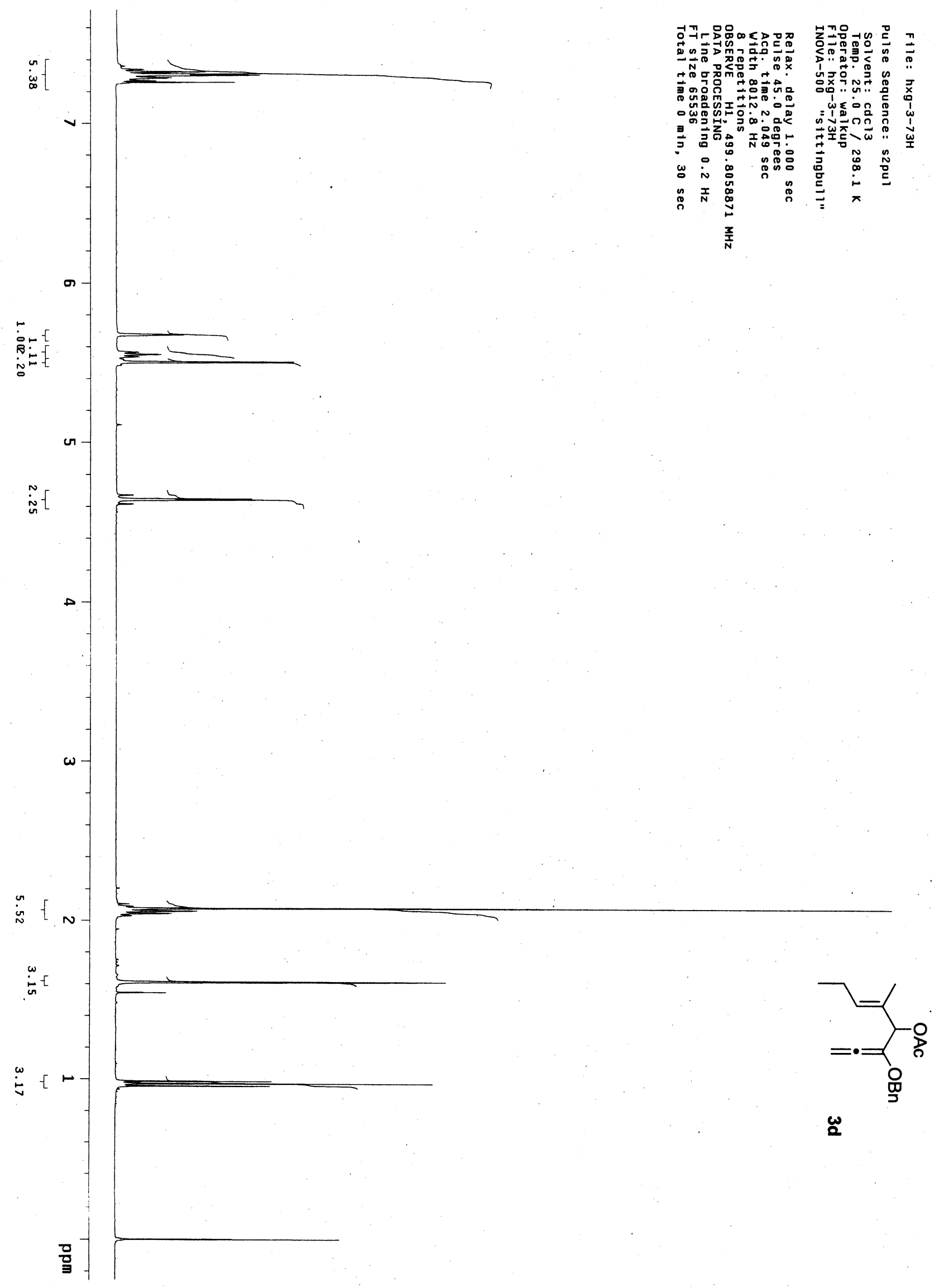



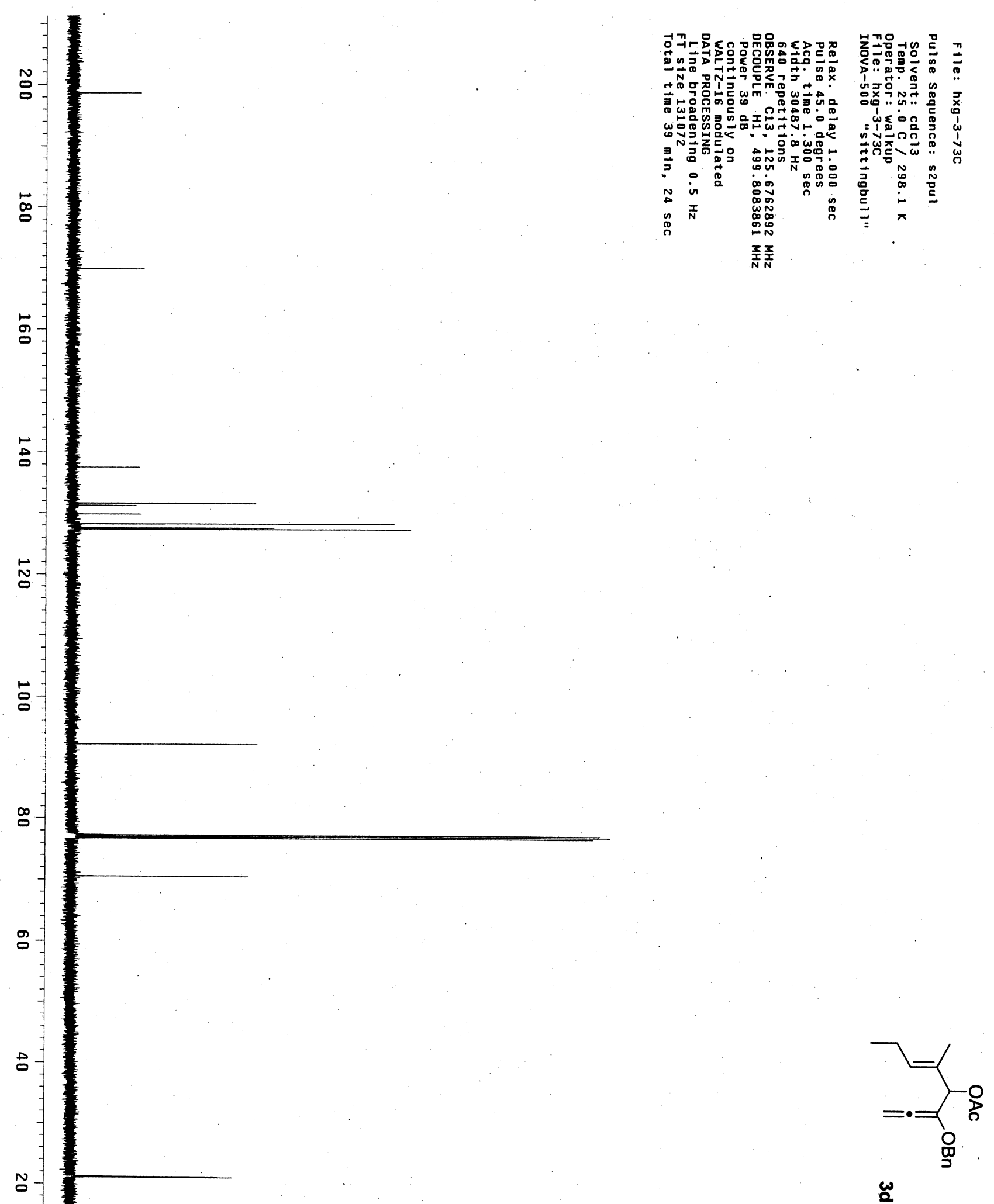


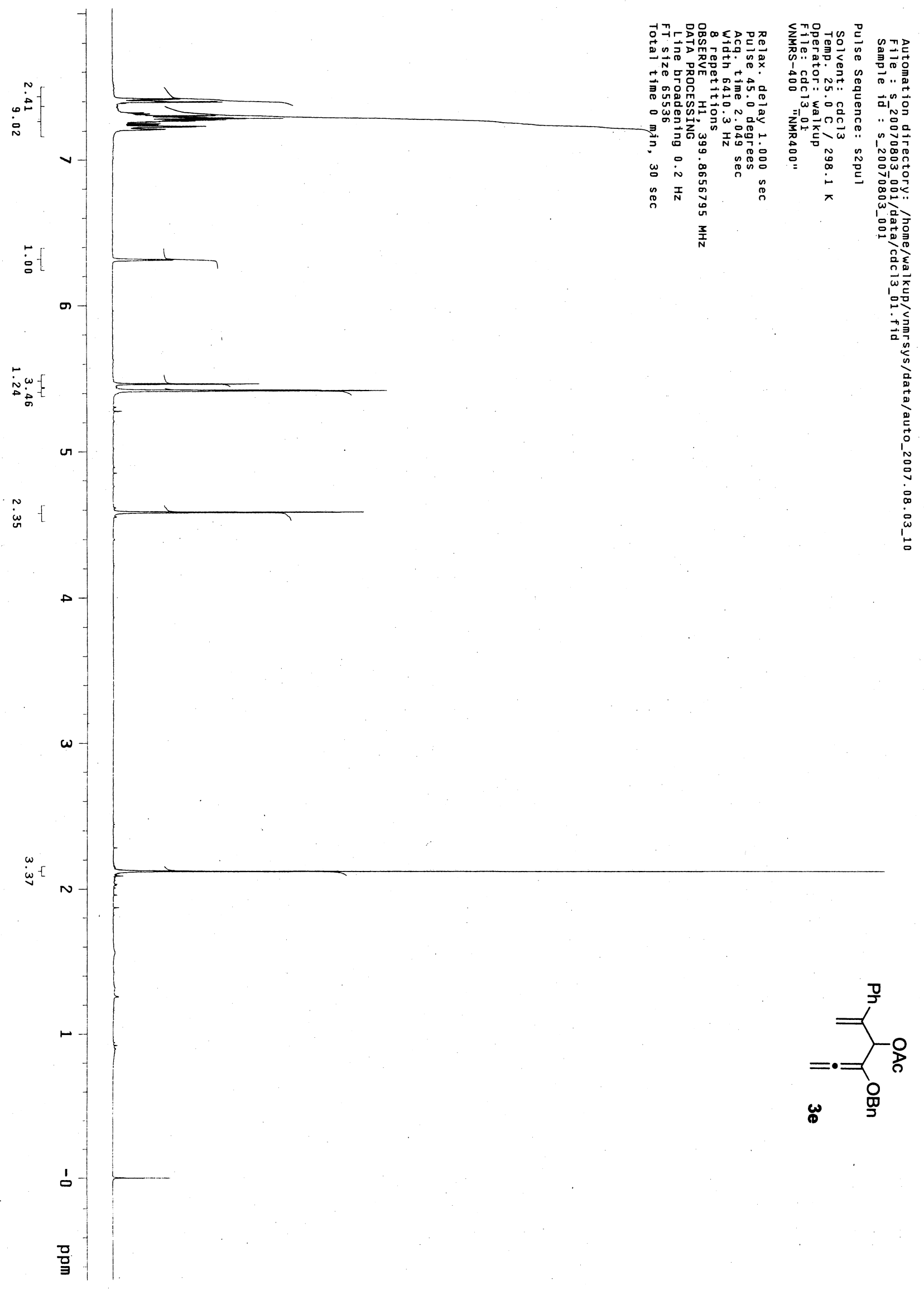



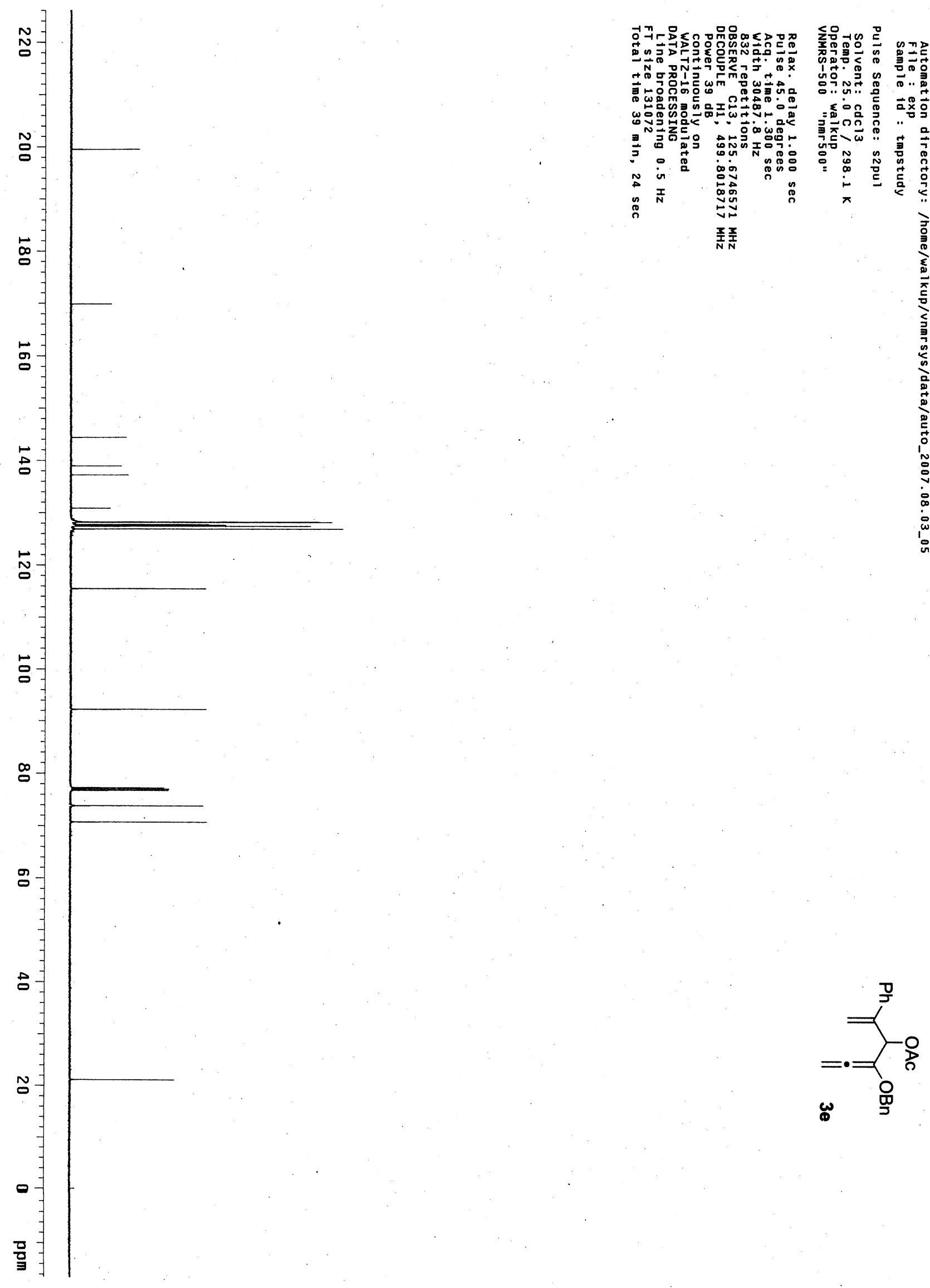

वे

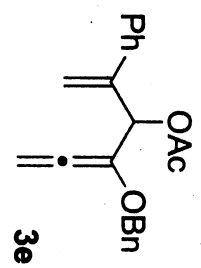




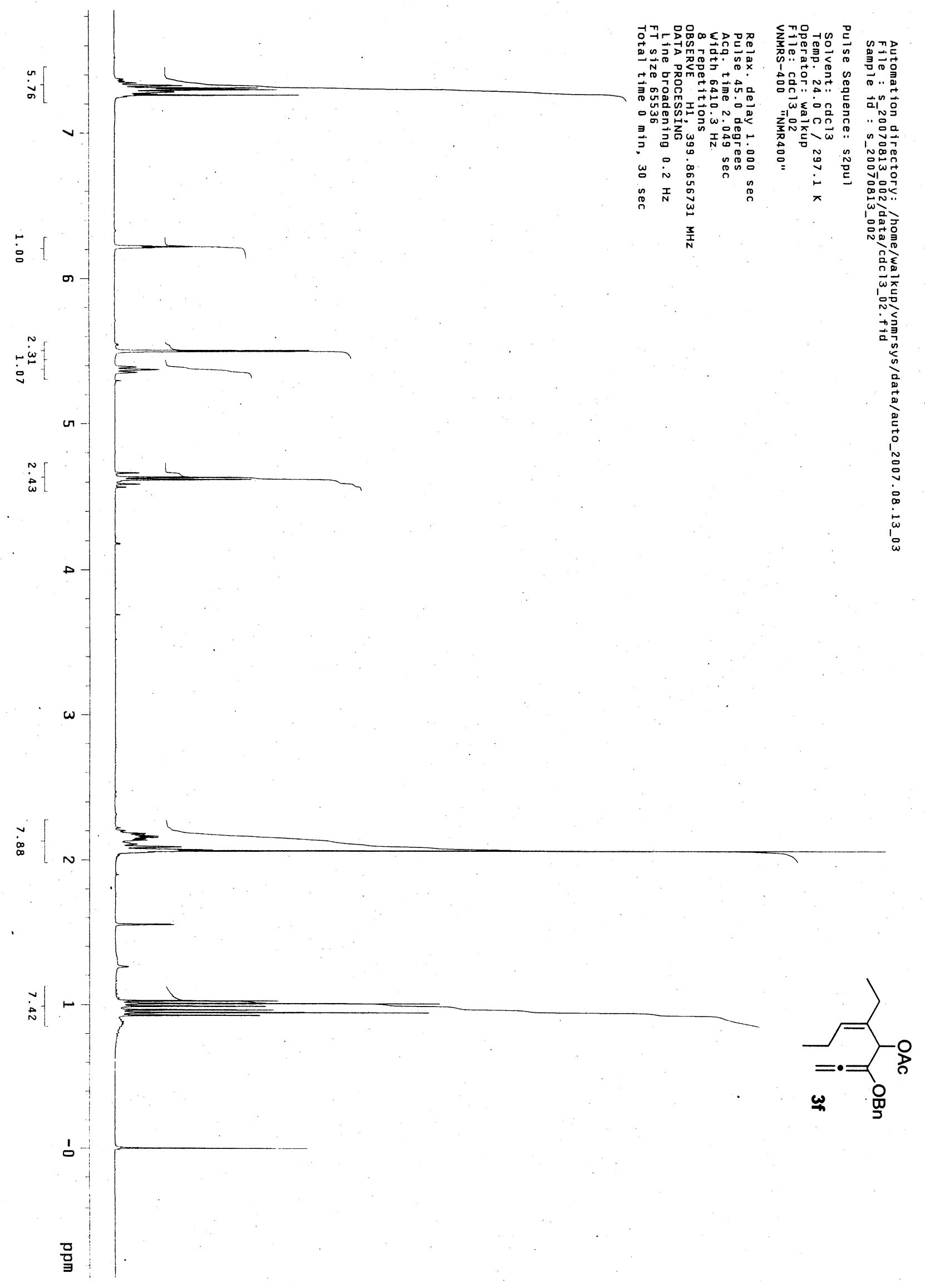




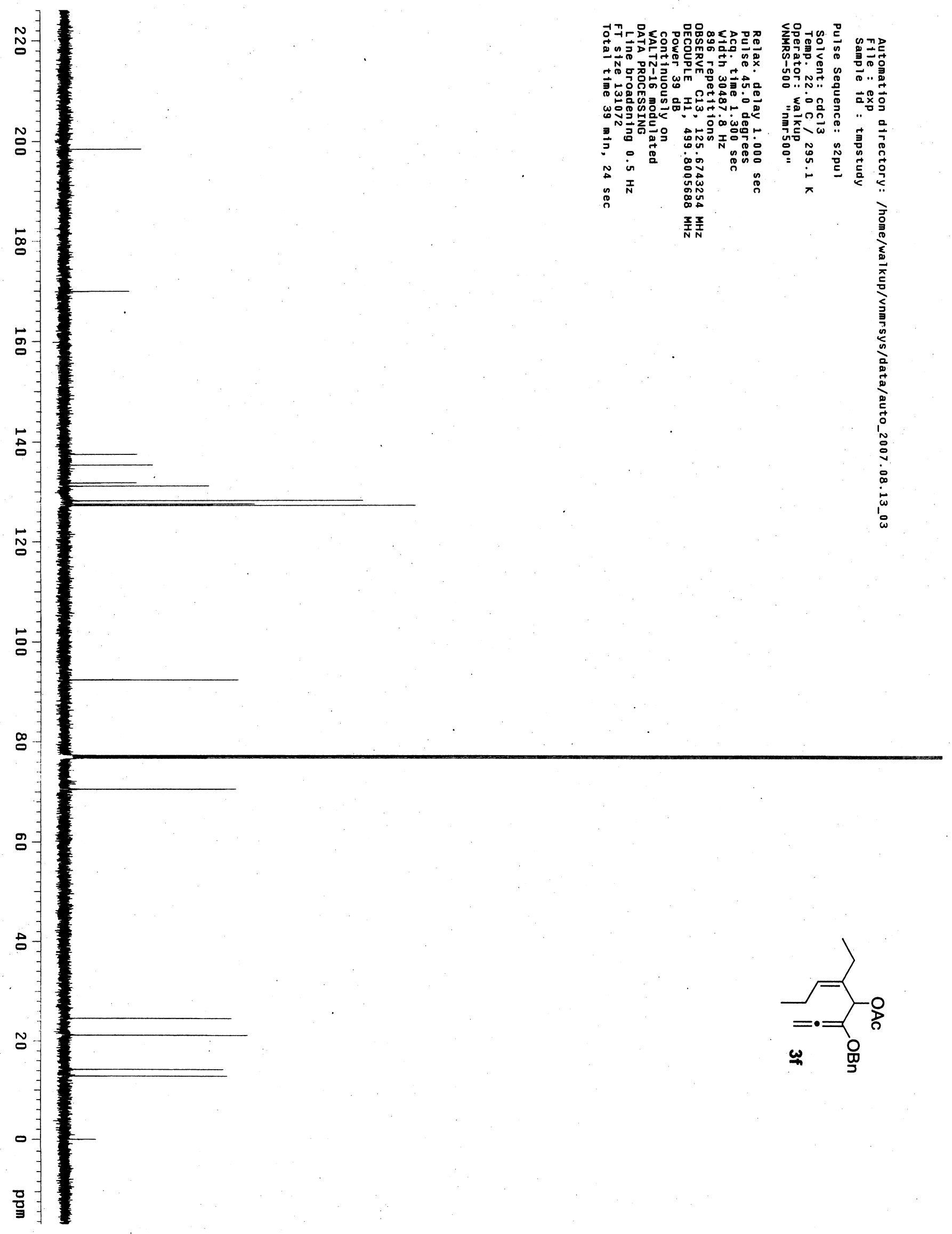



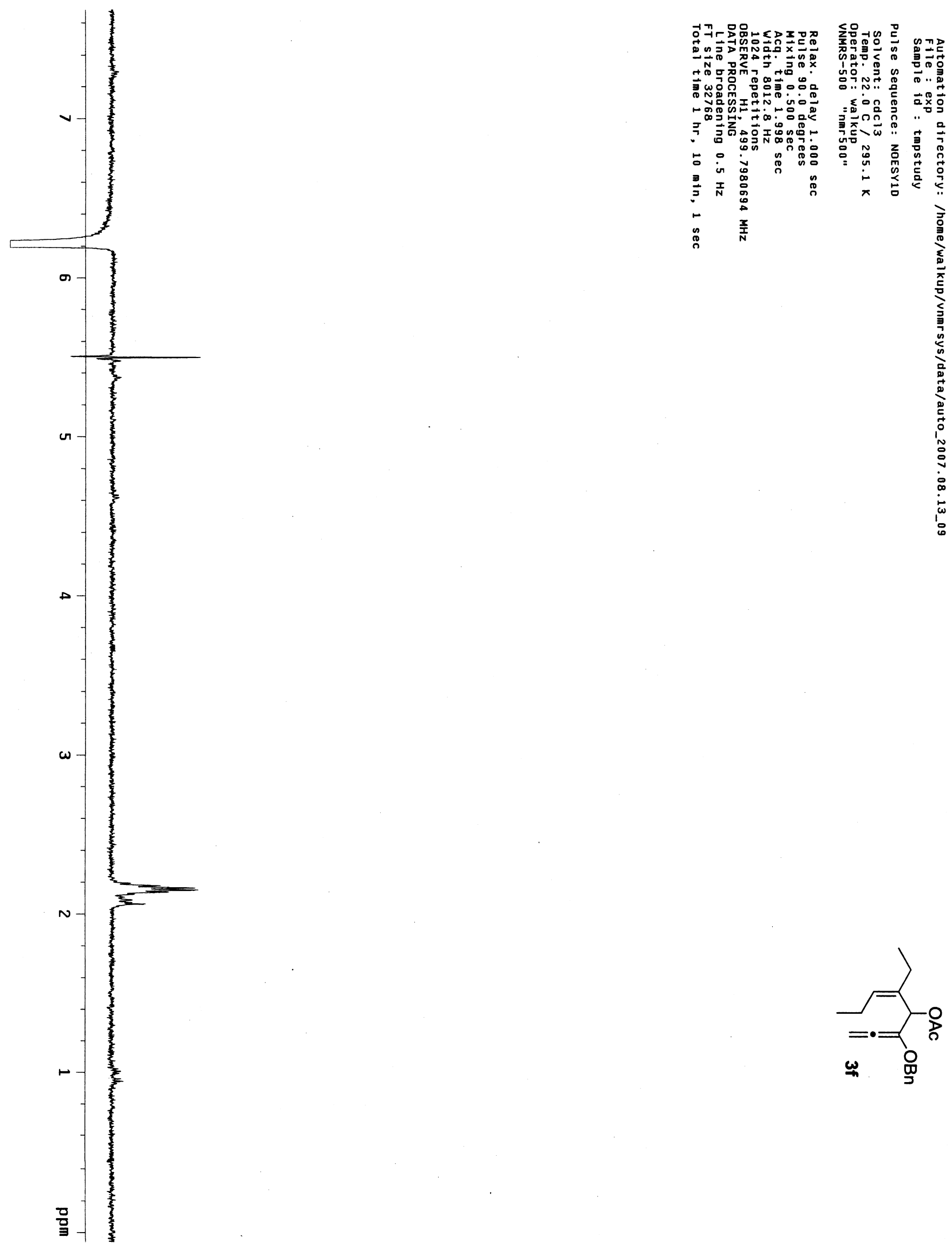


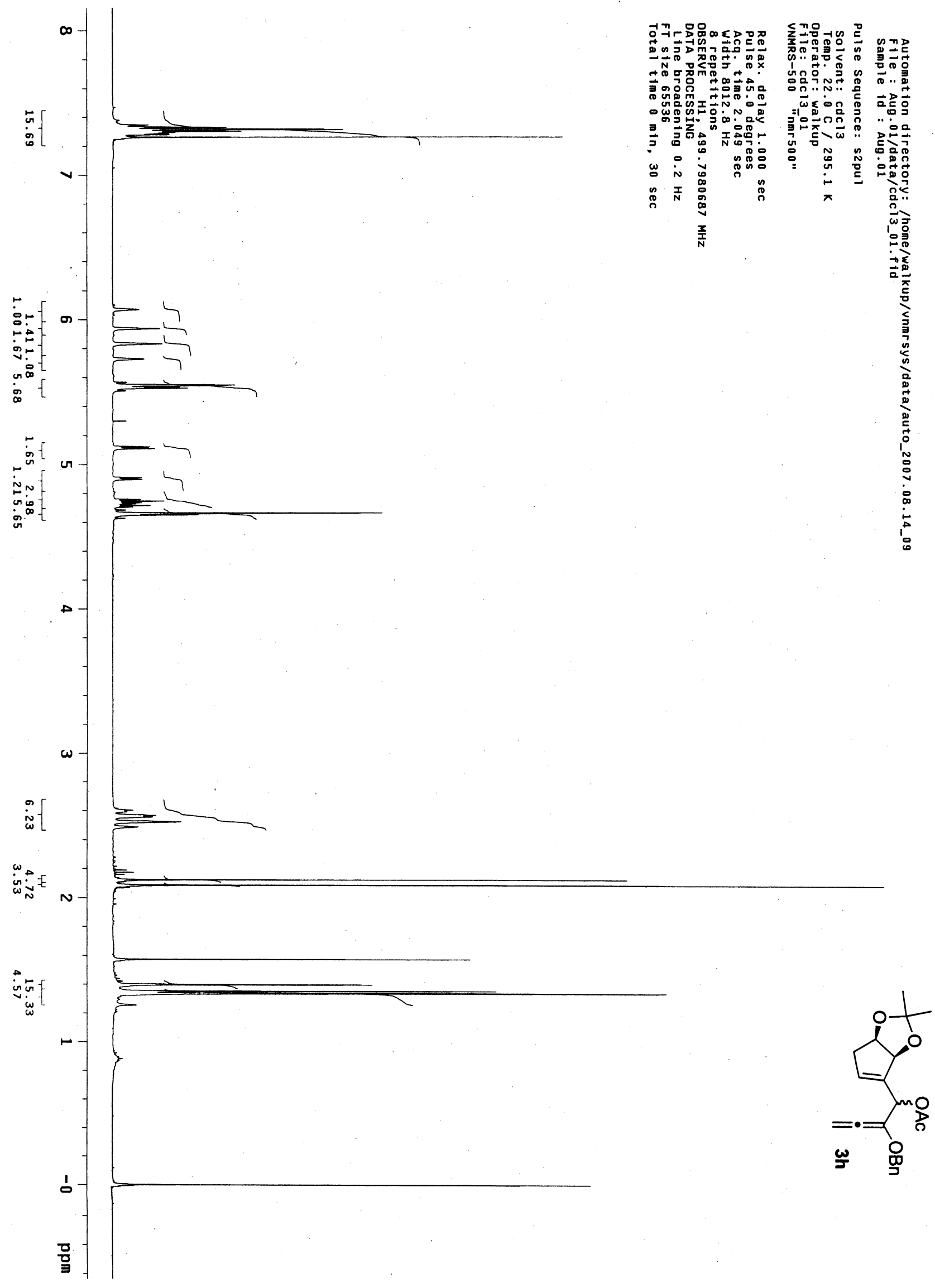




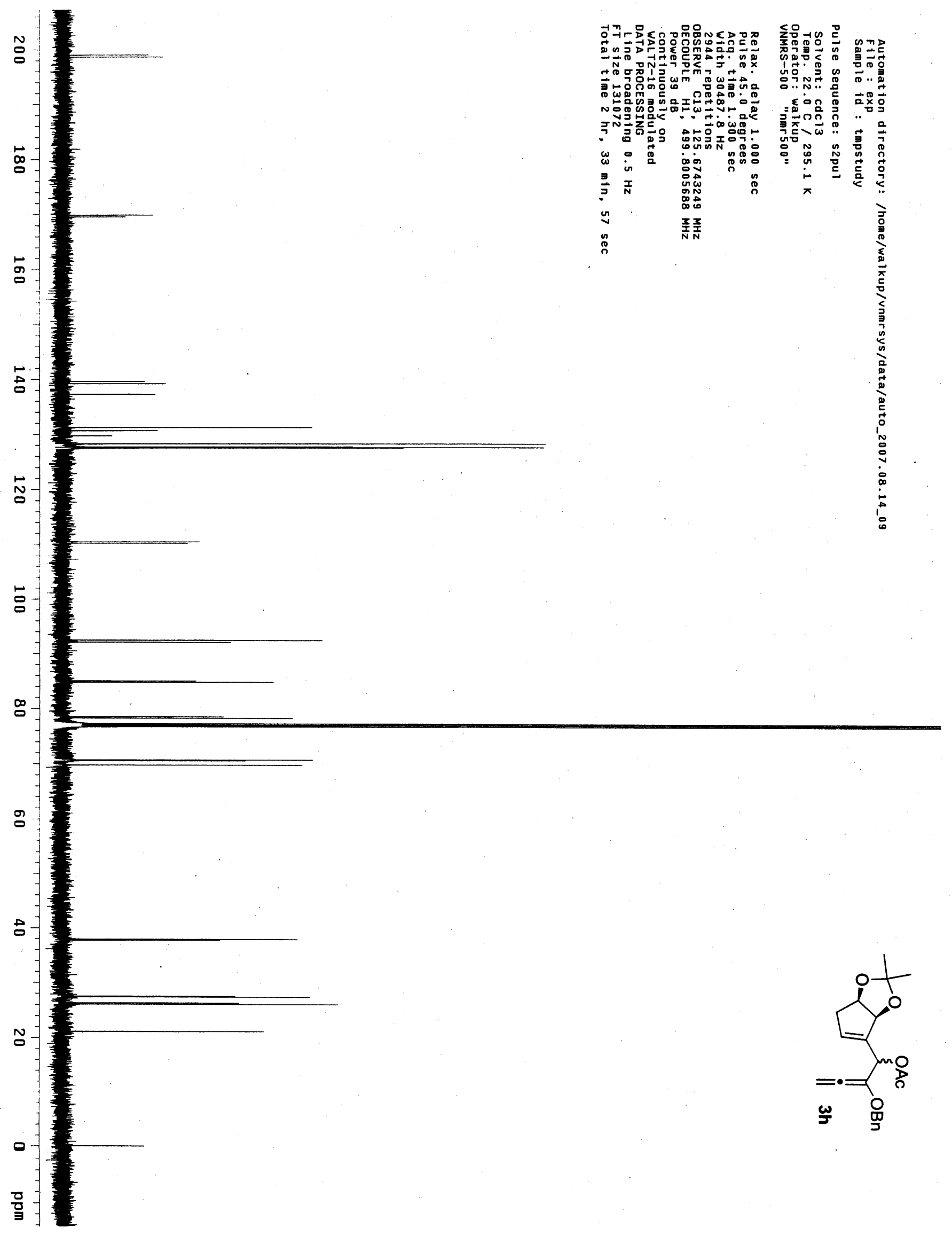



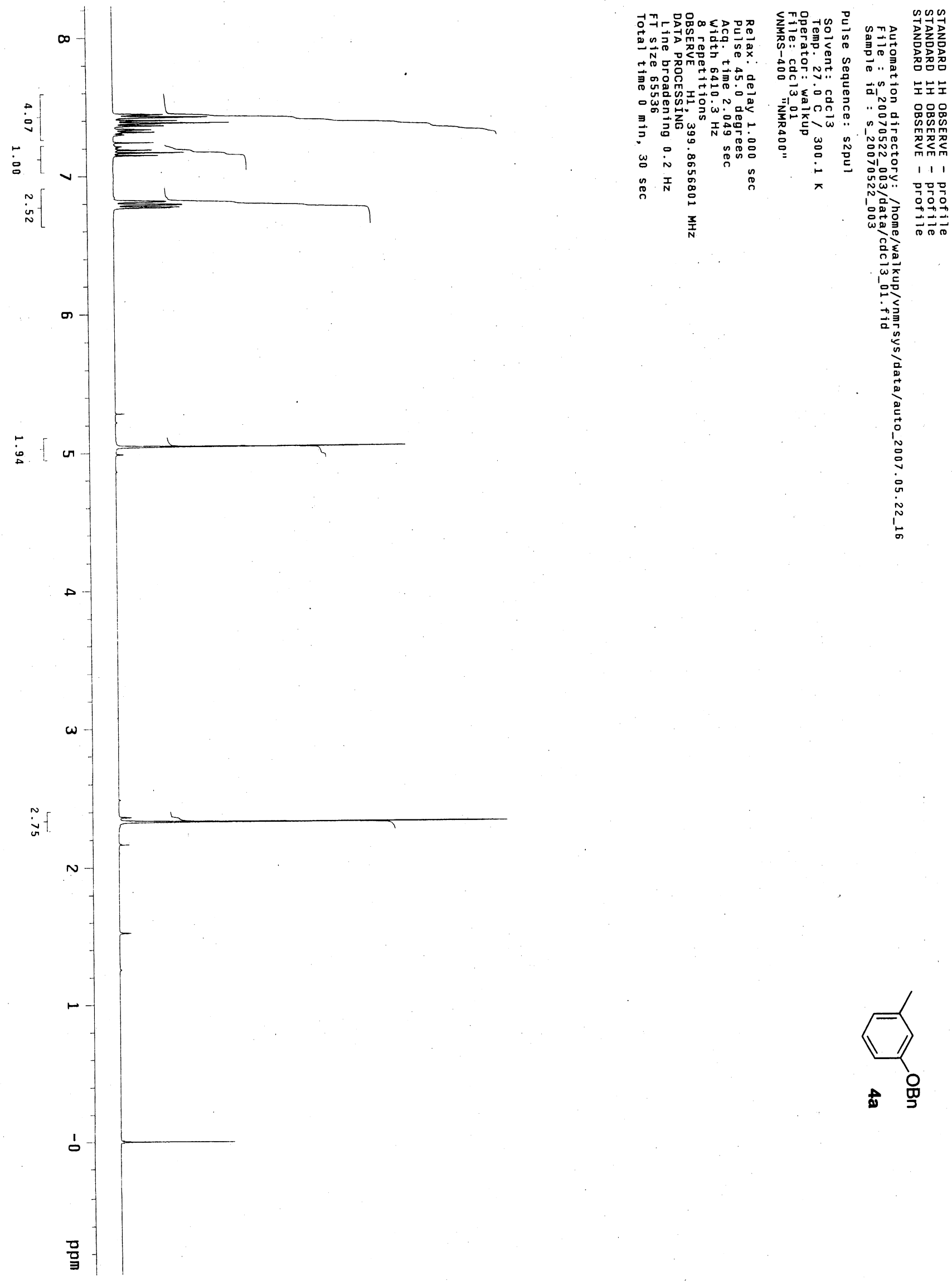

=1

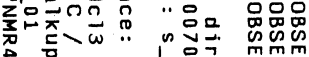

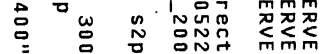

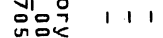

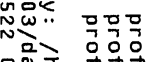

造记

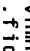

0
0
0
0
0
0
0
0
0
0
0
0
0
0
0
$\tilde{N}$
0
0
0

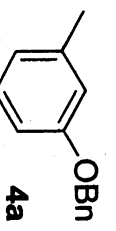



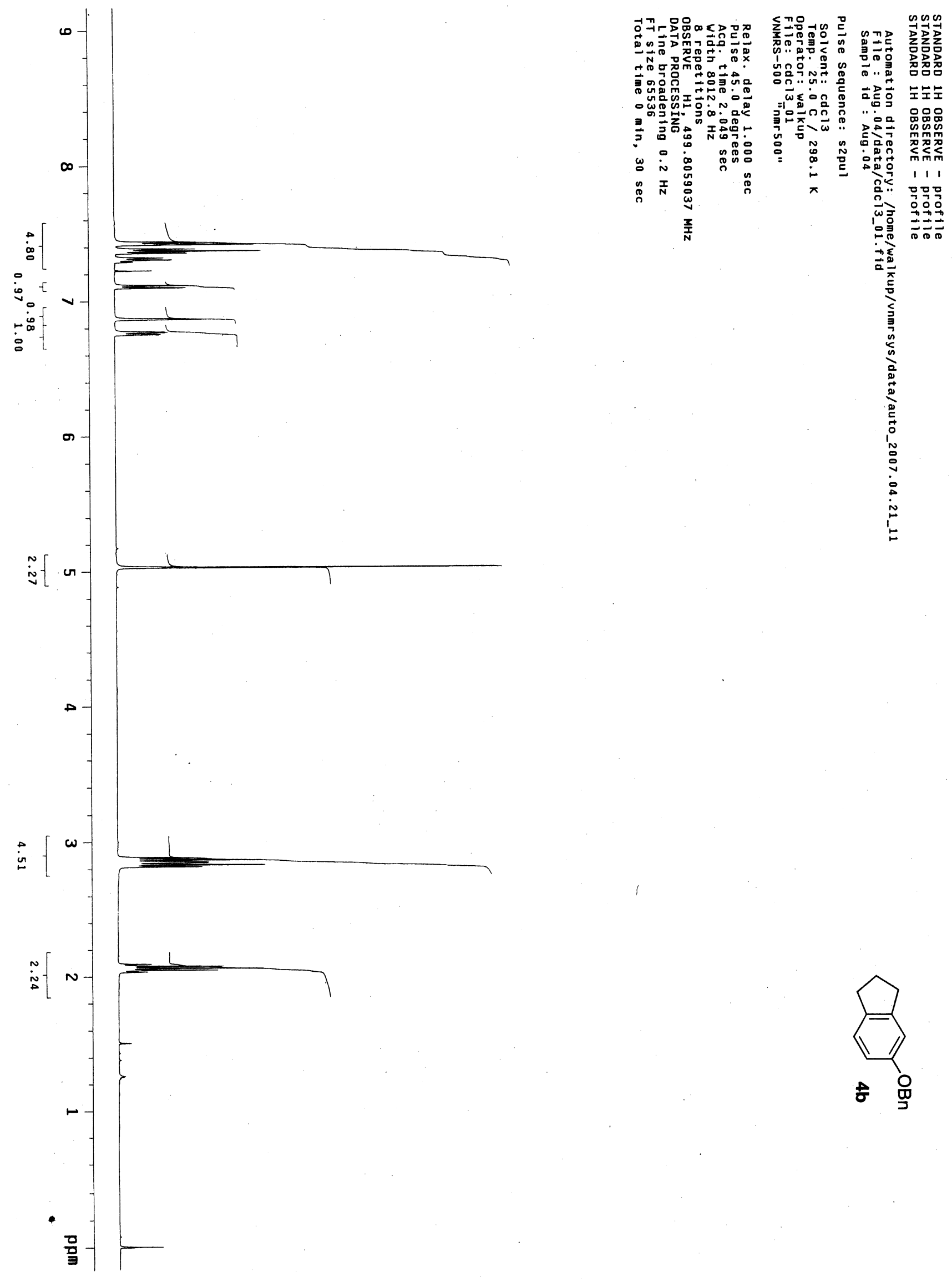

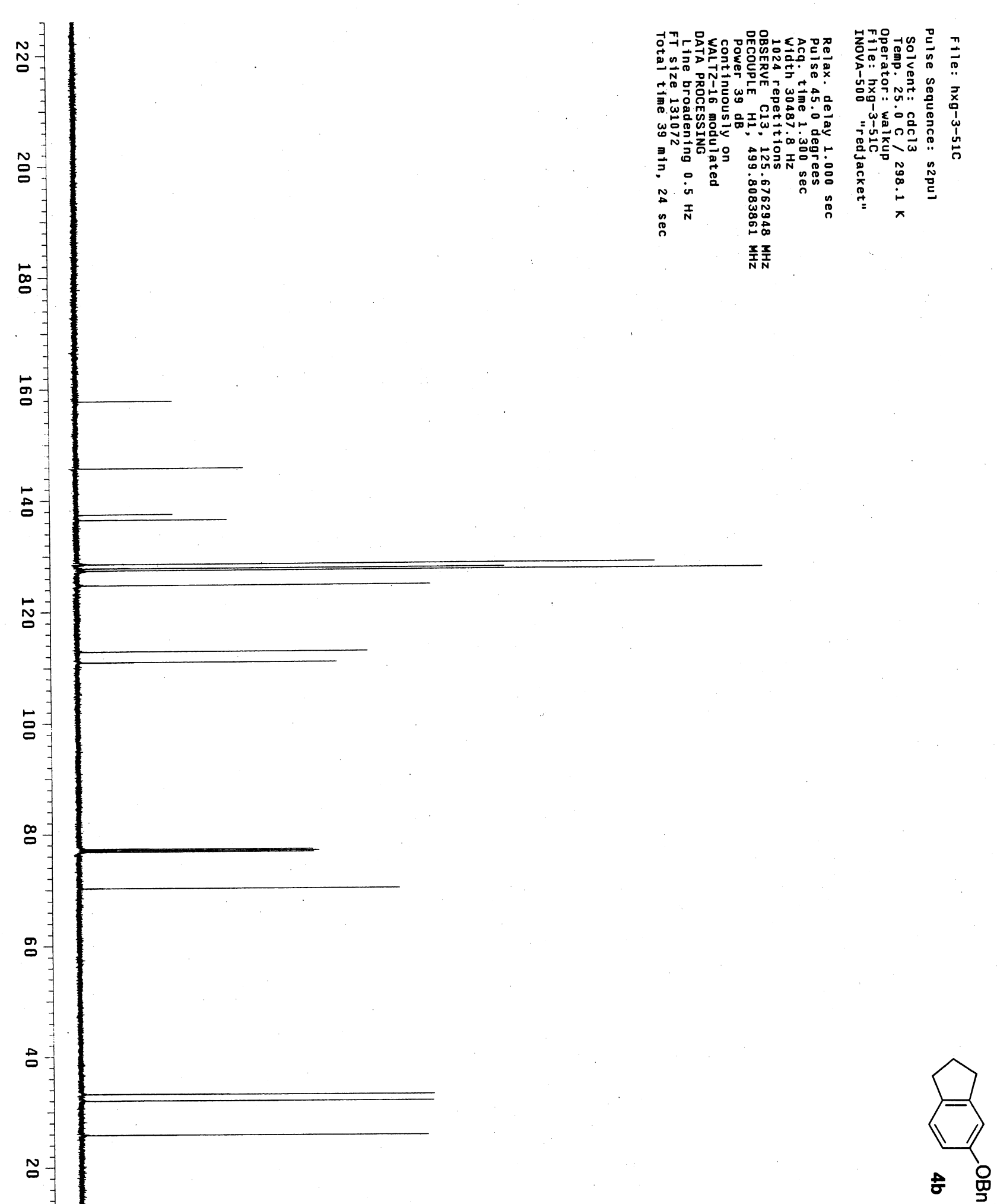

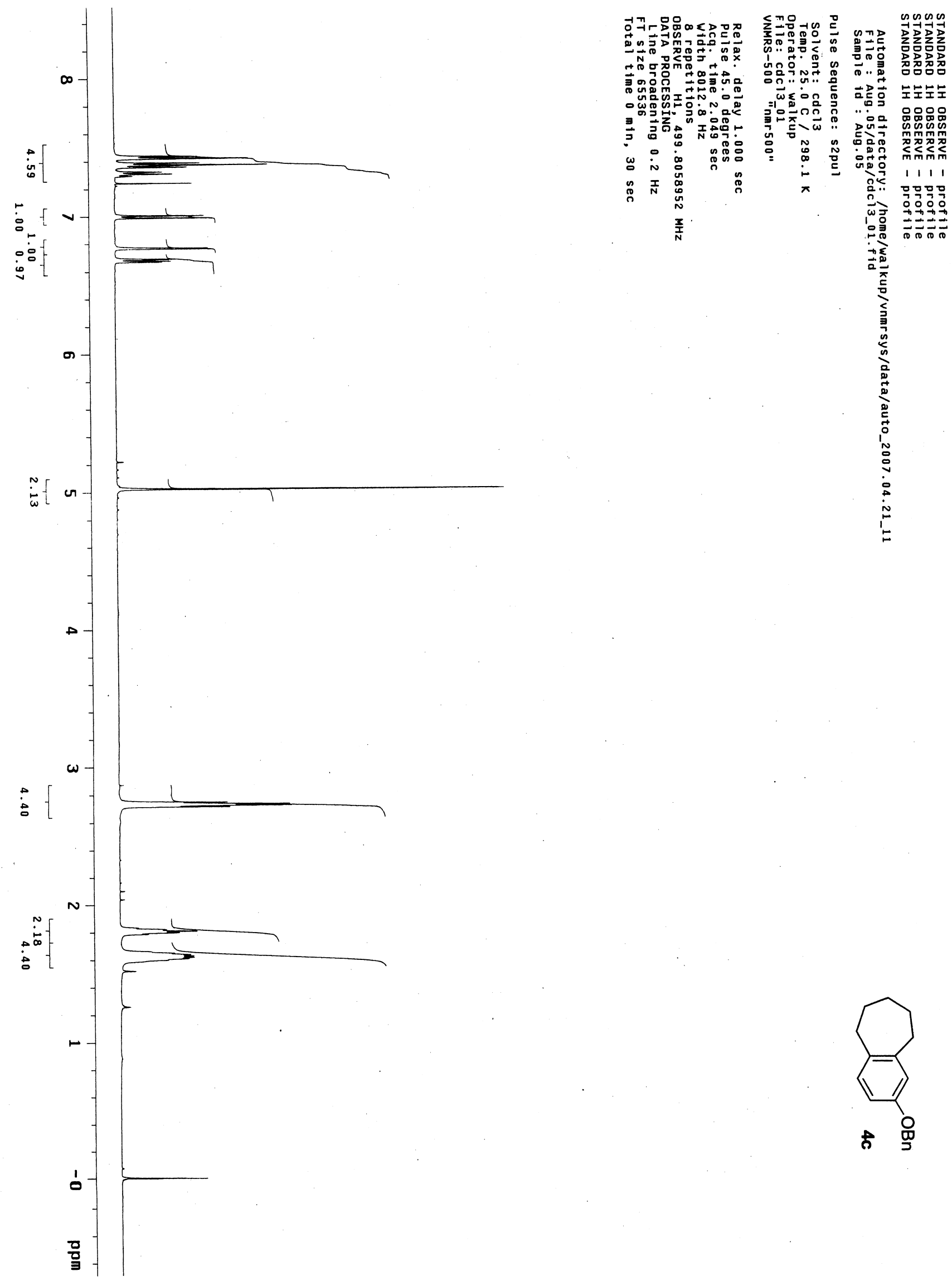

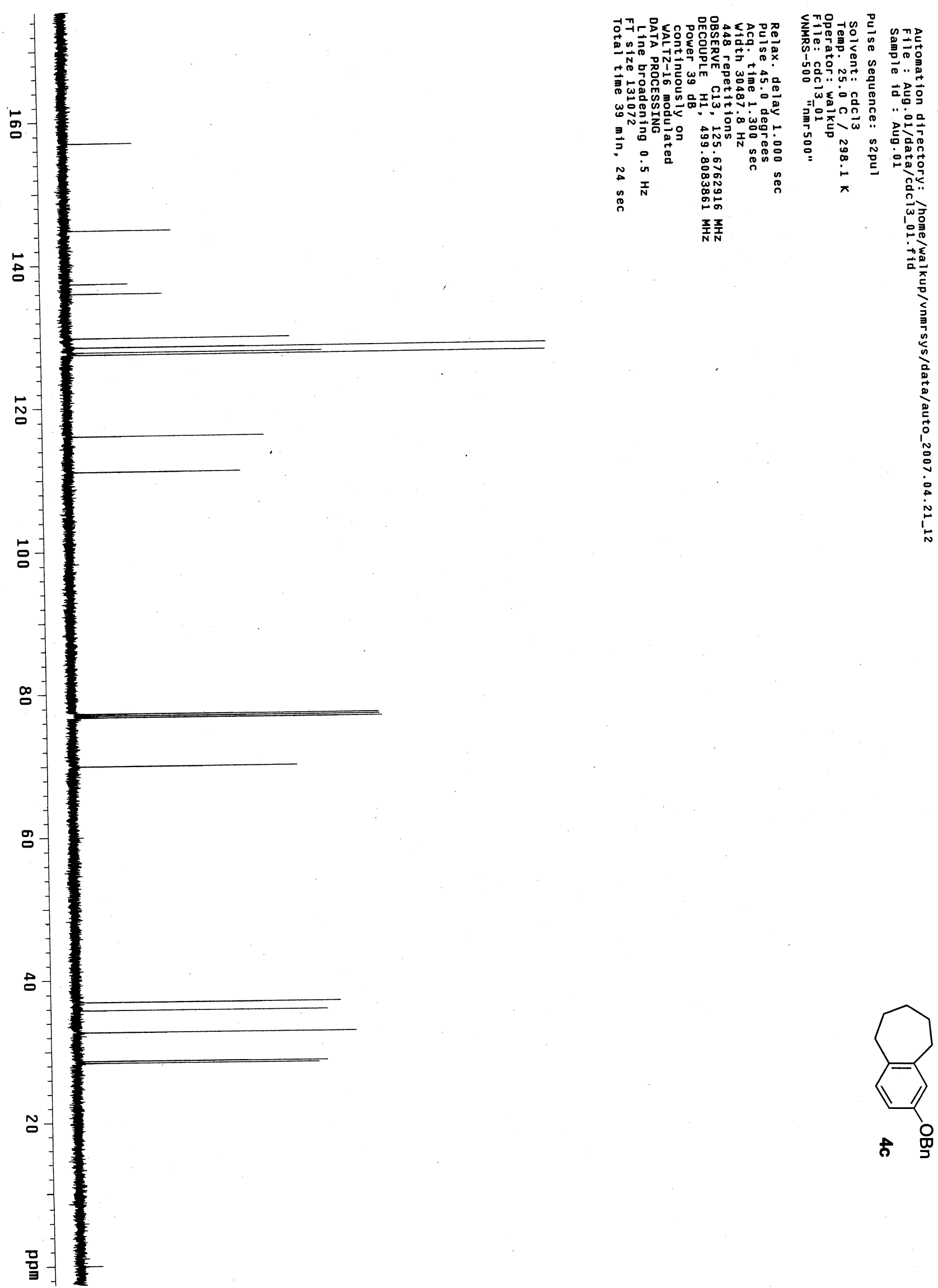


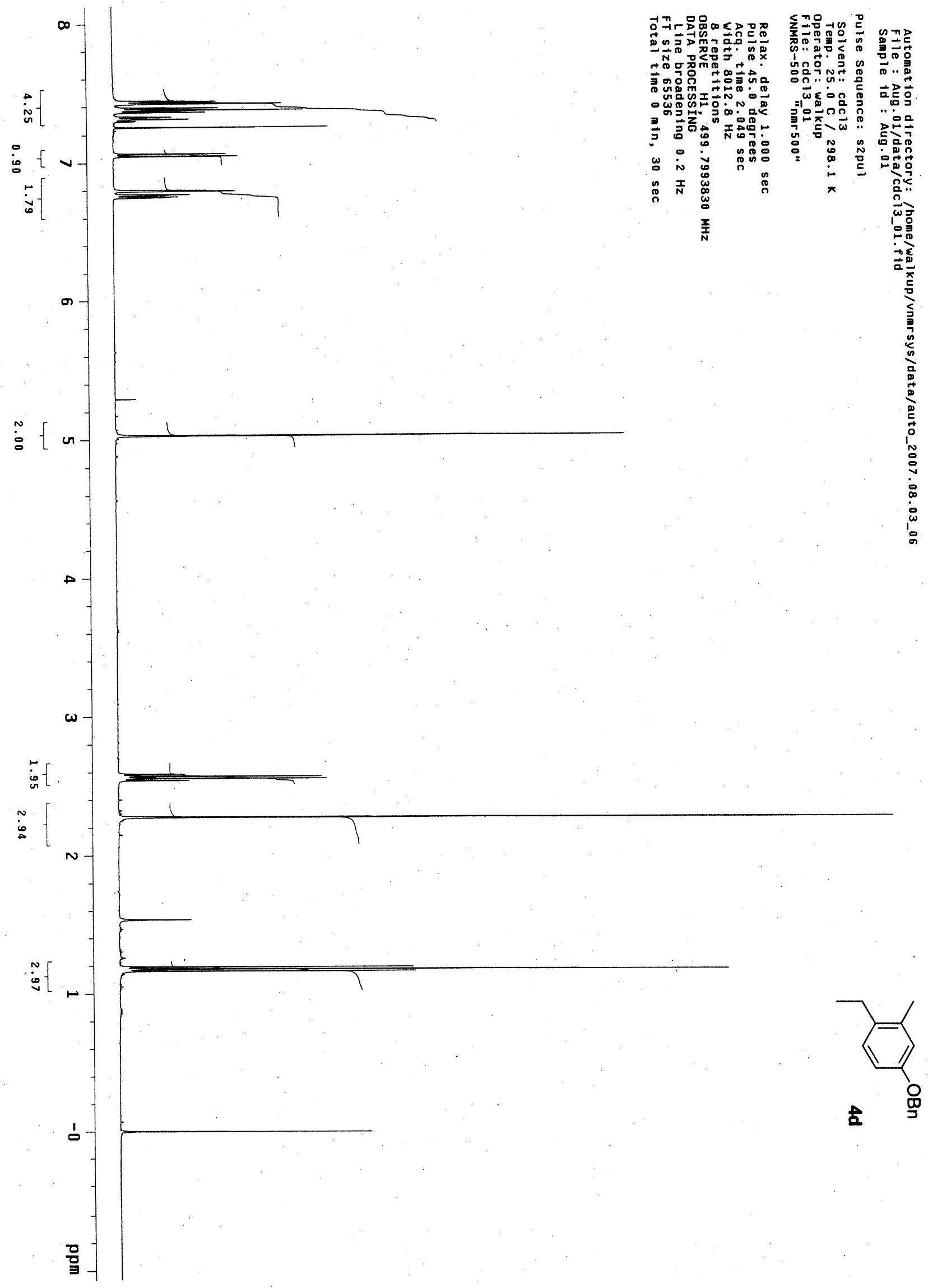



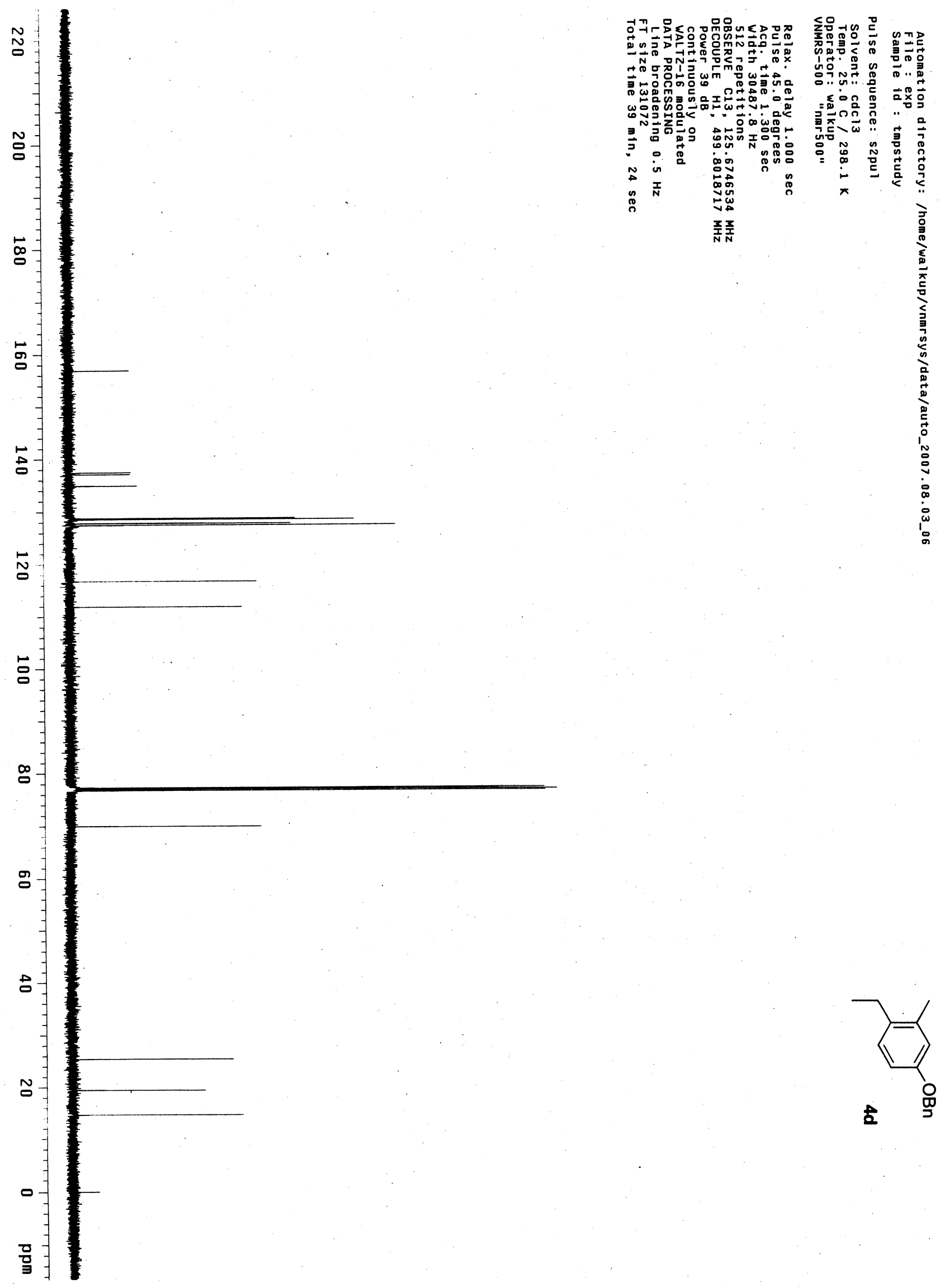

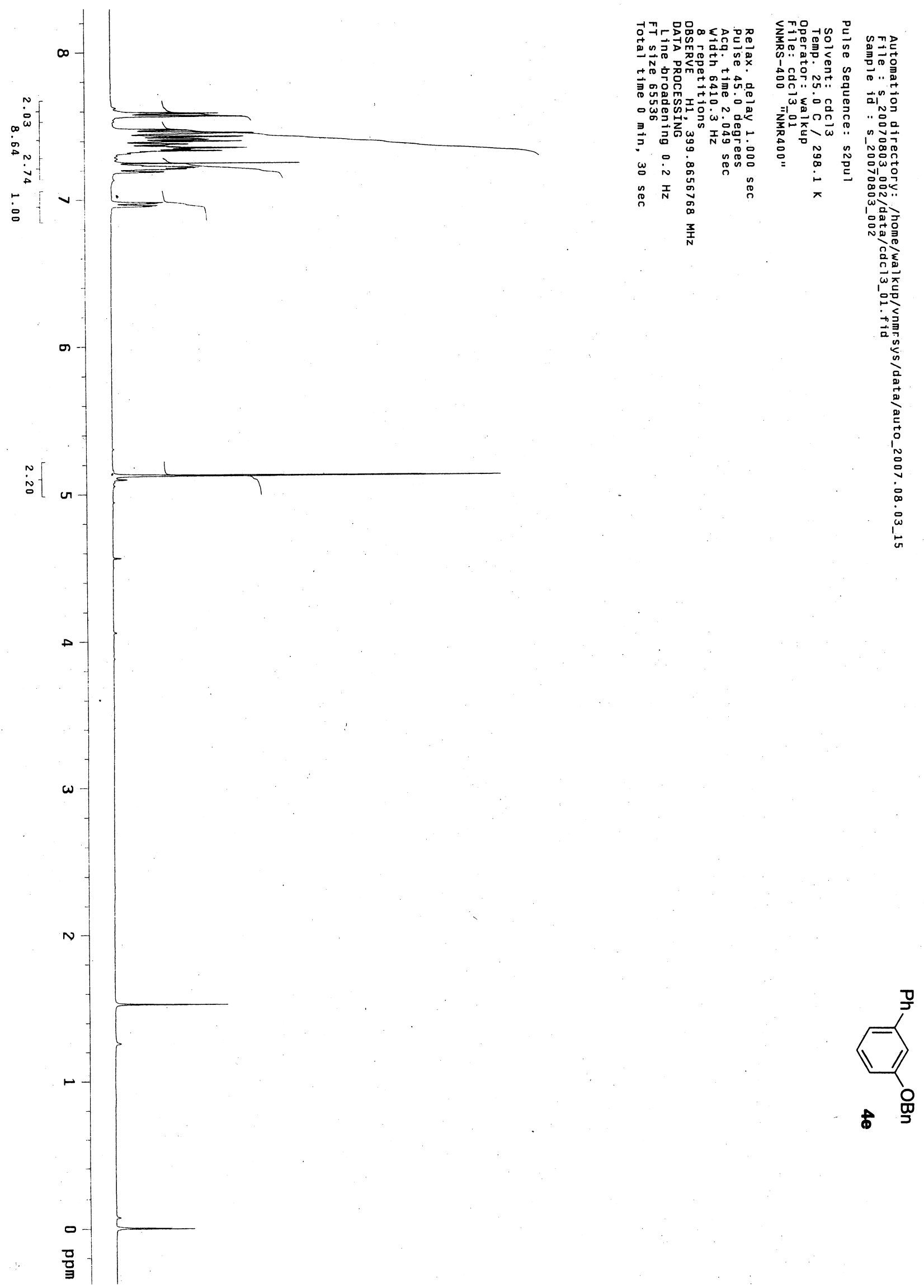

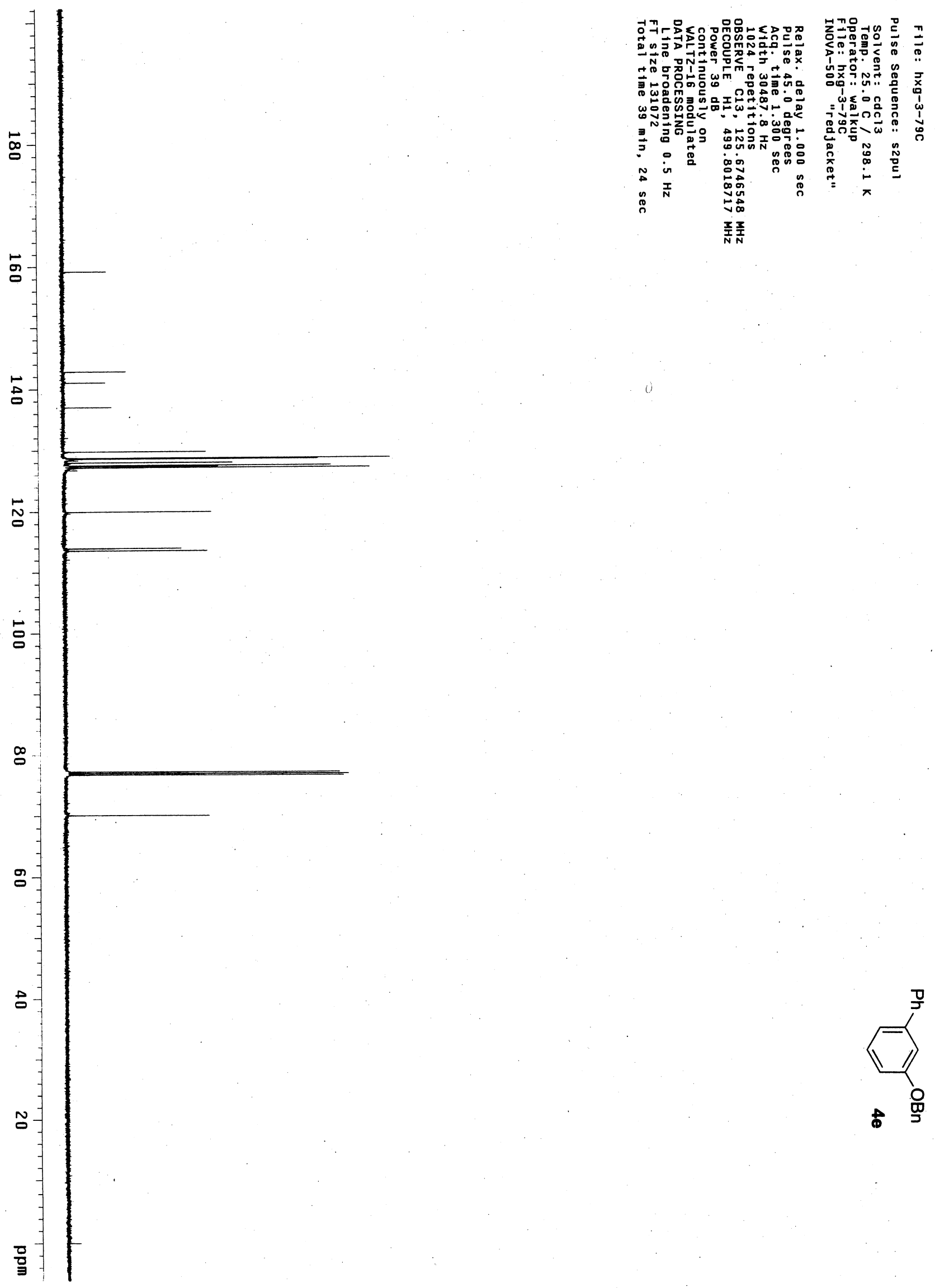


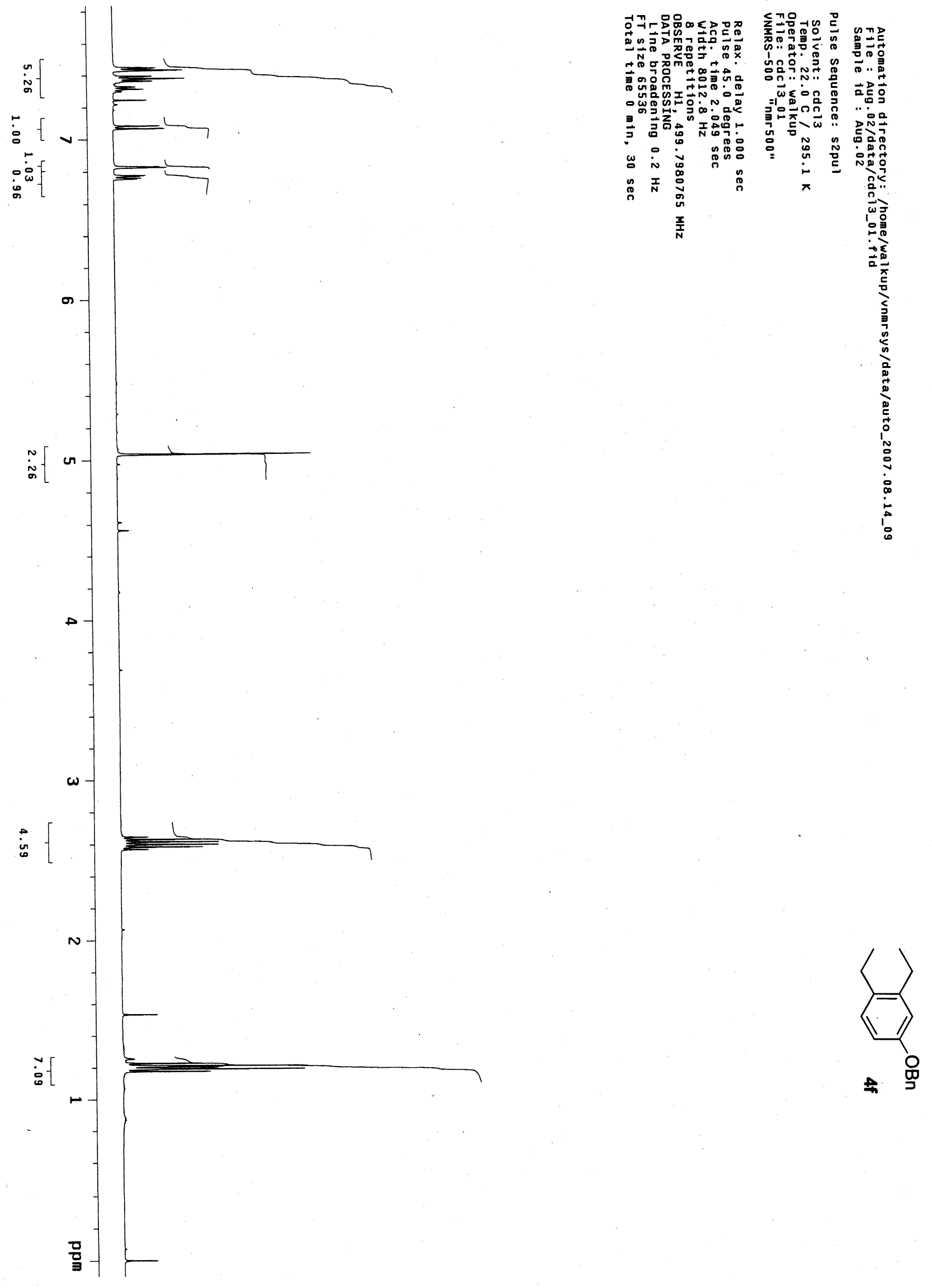



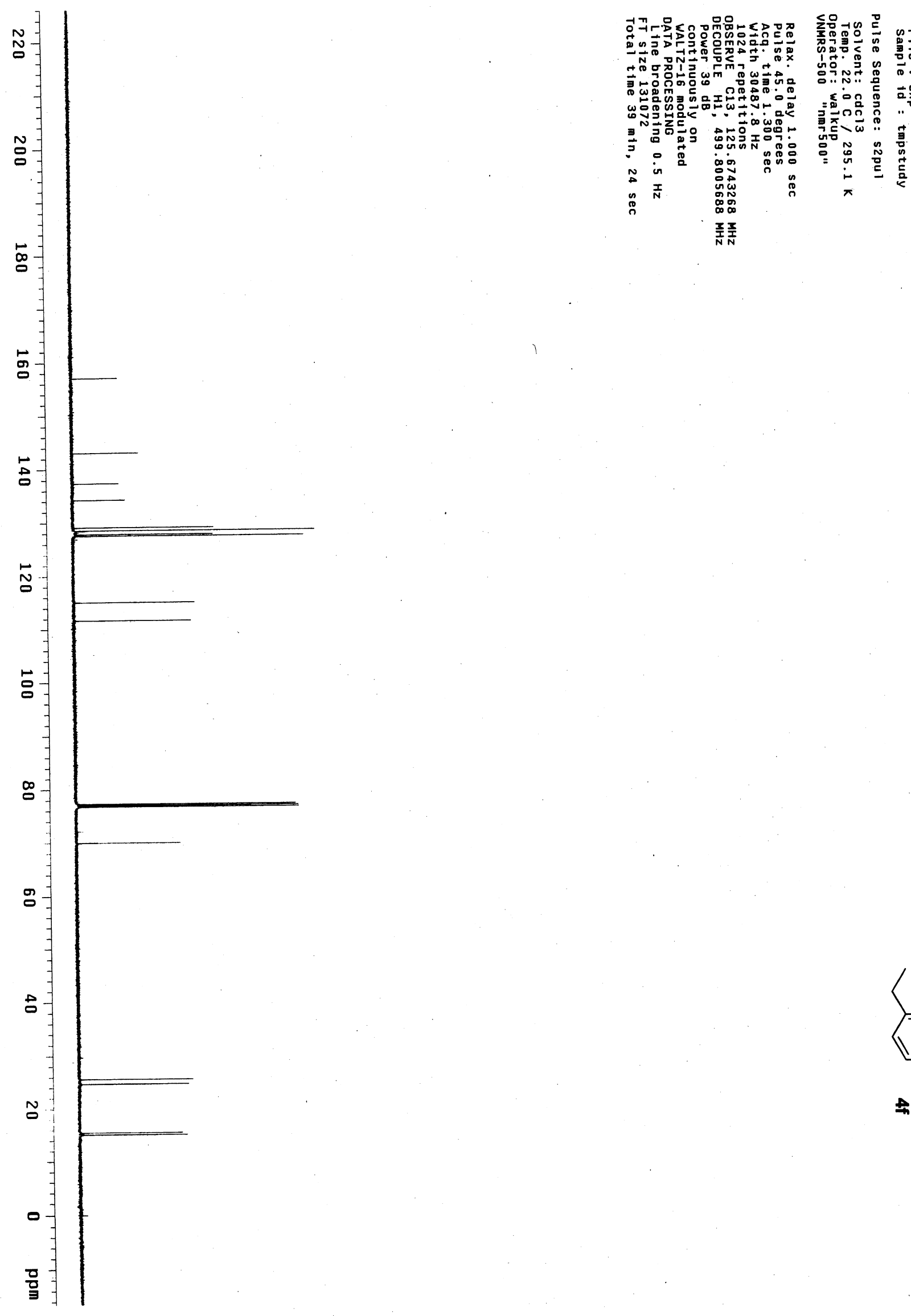

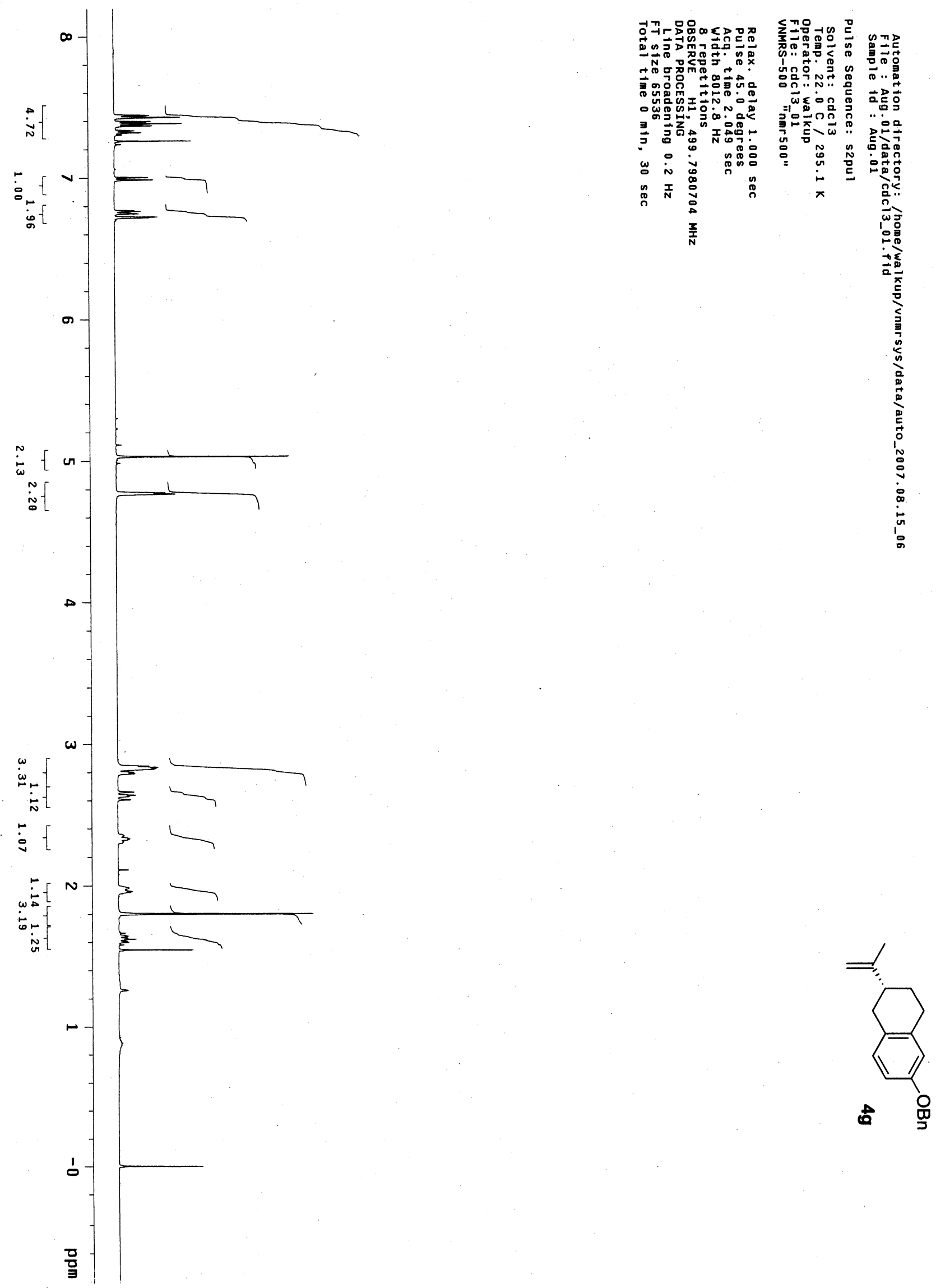


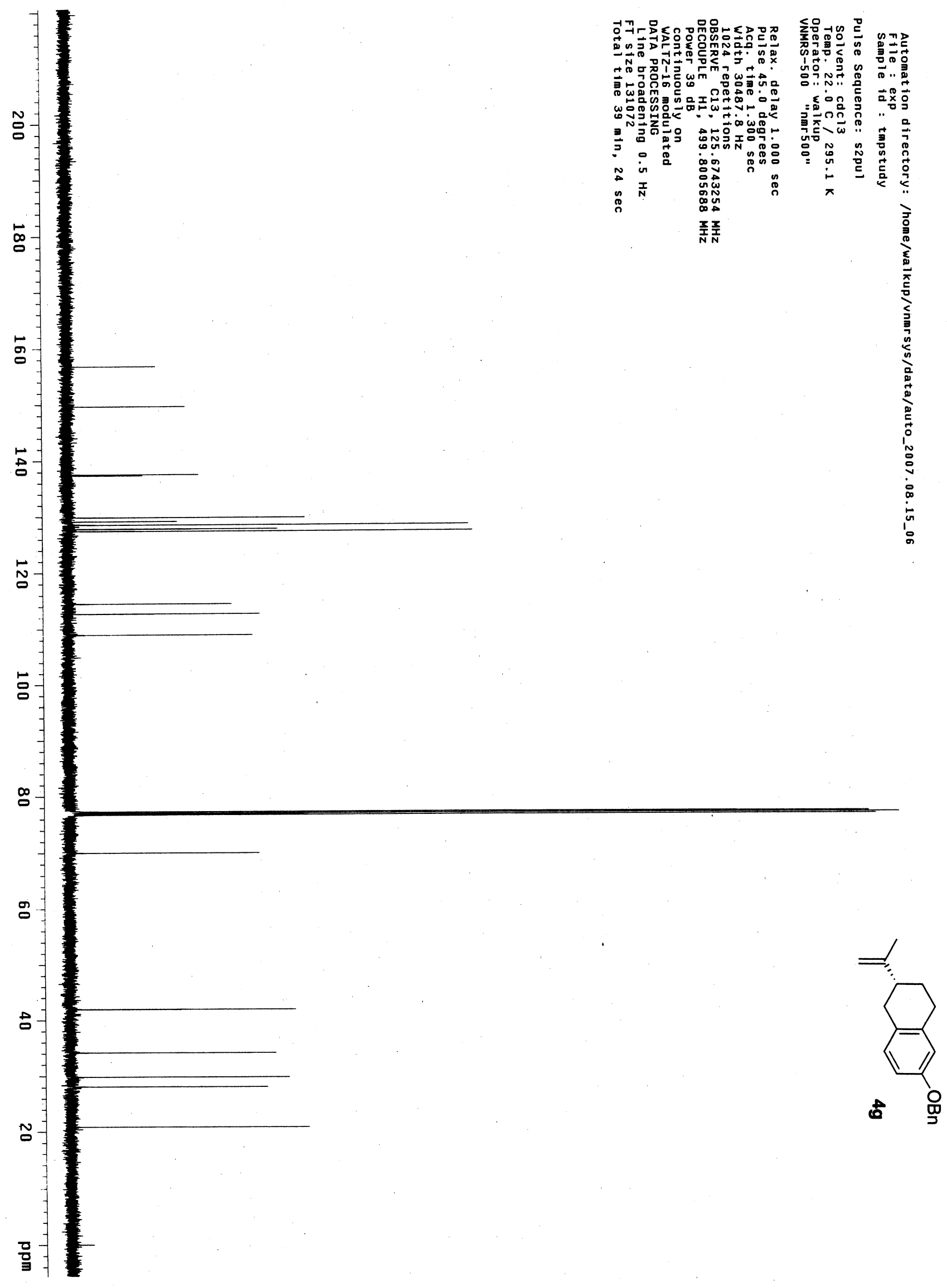



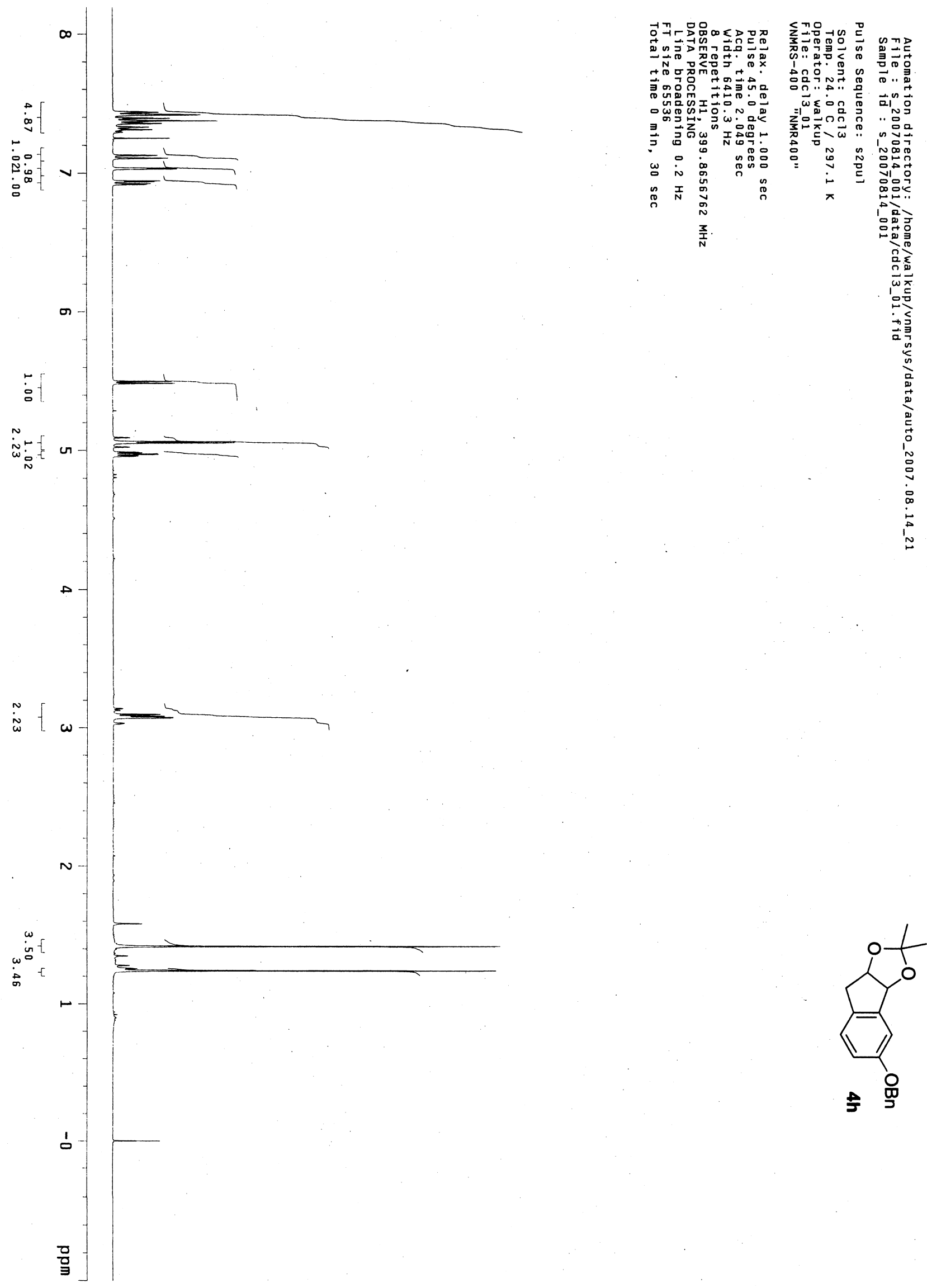

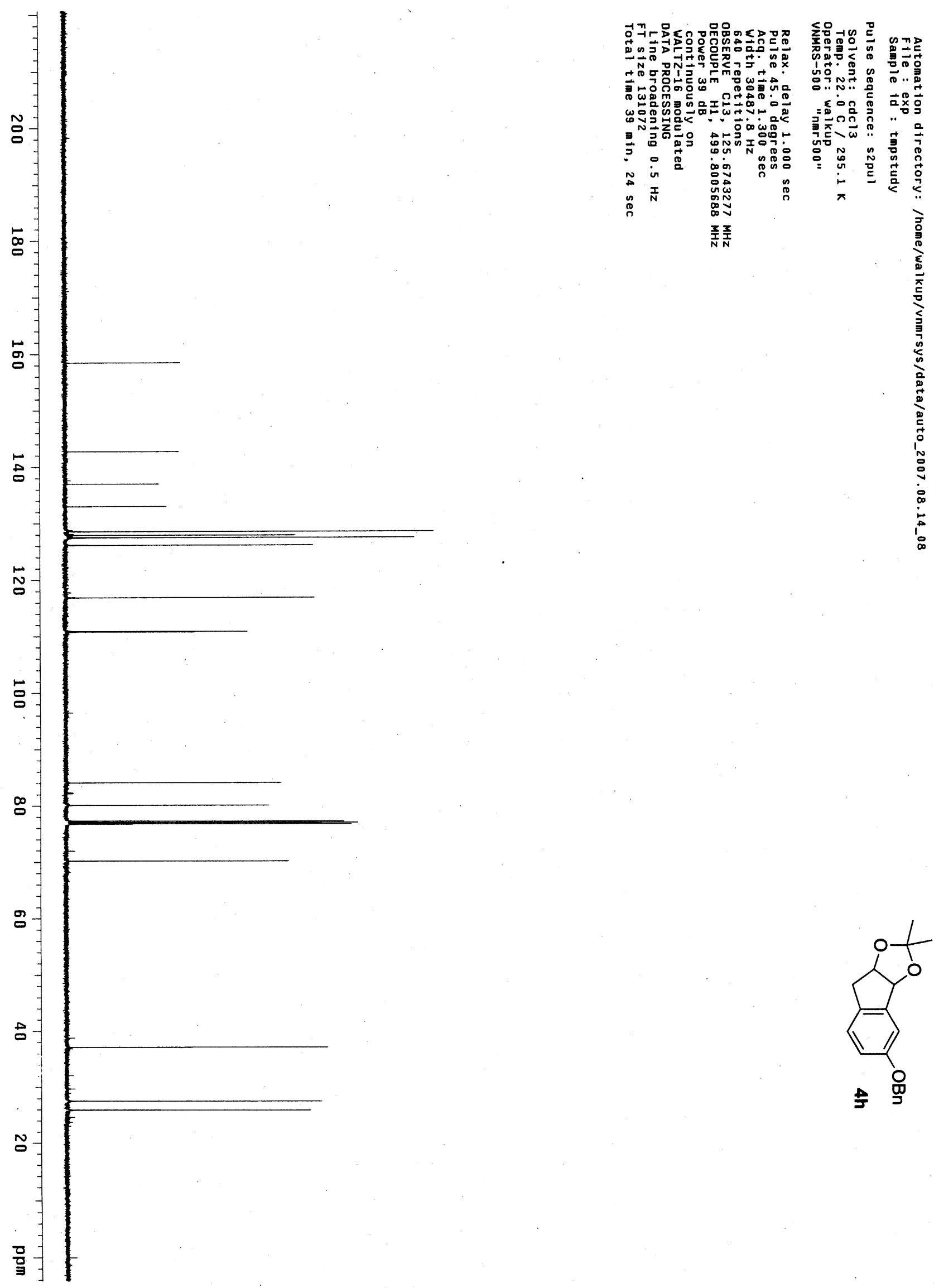

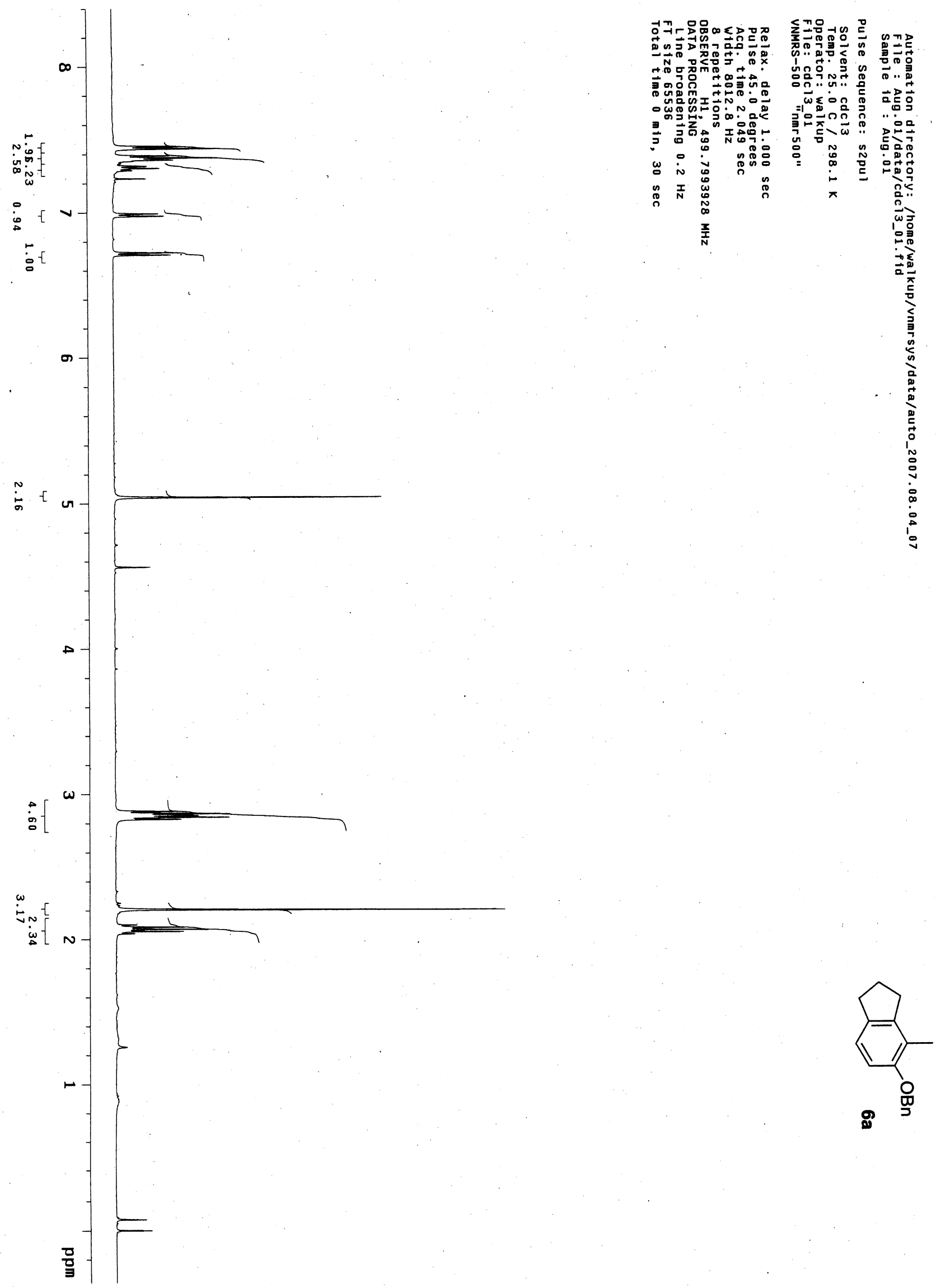

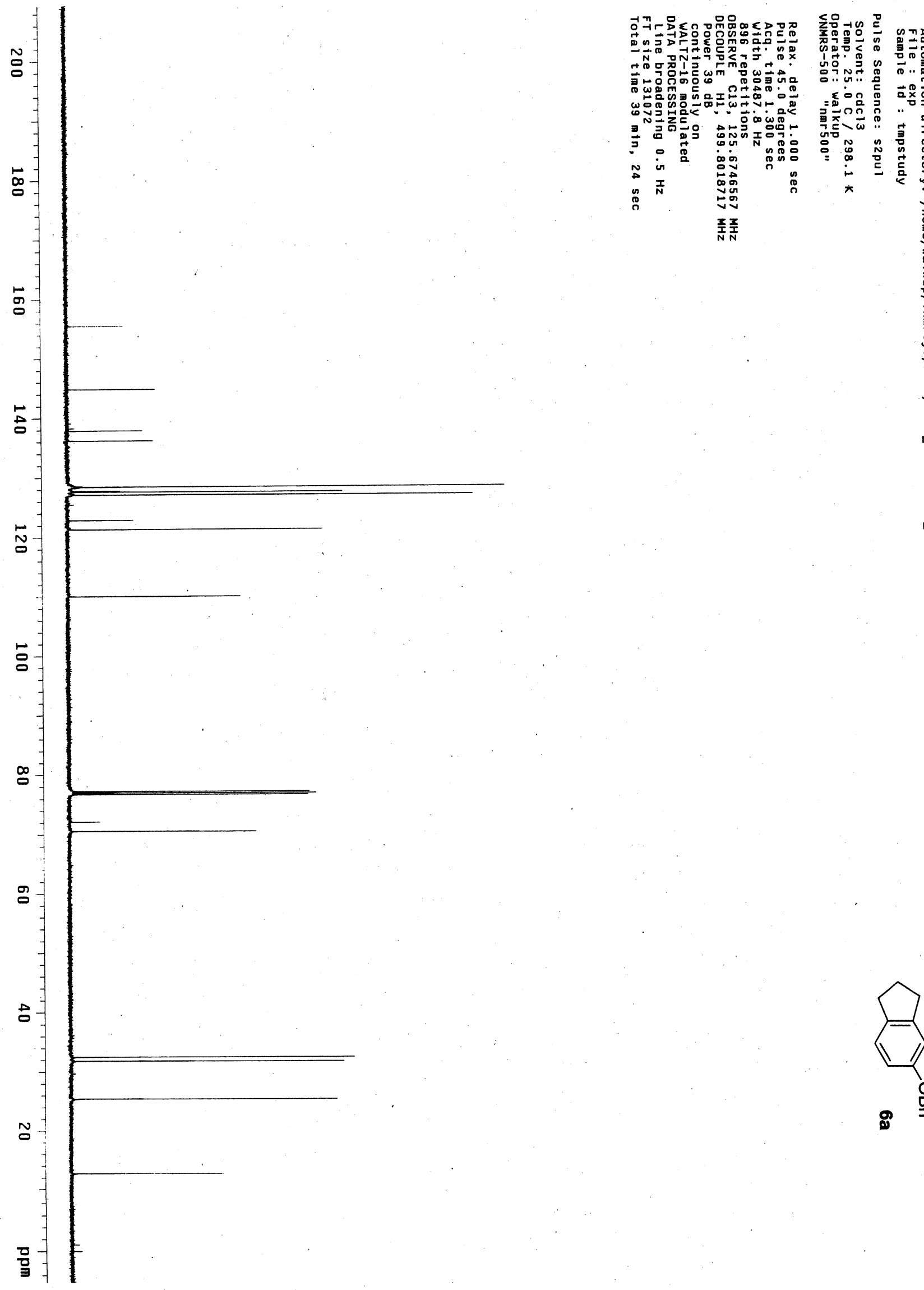

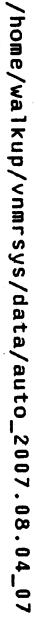

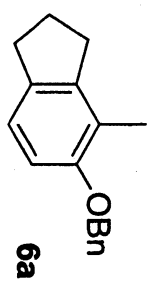




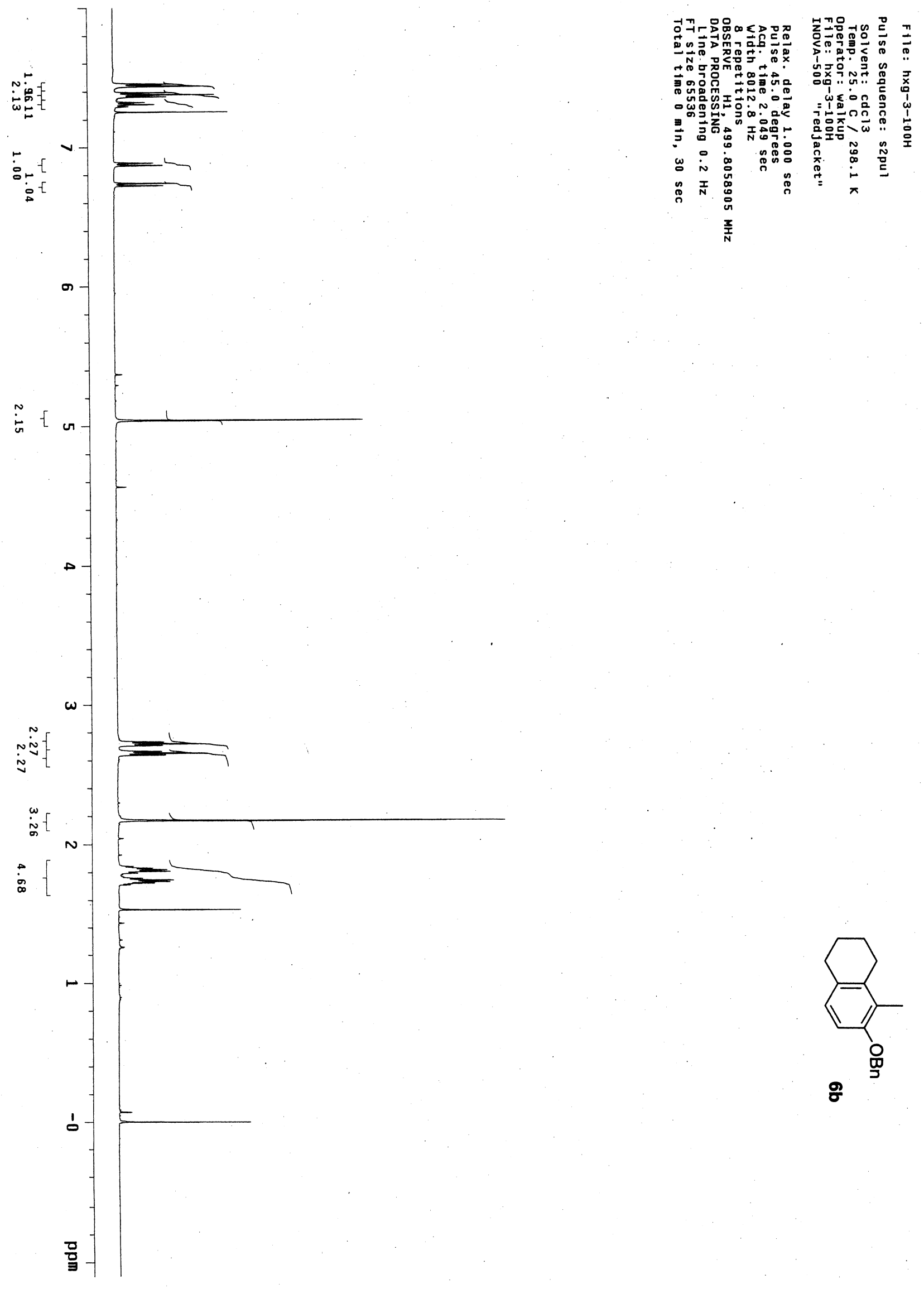




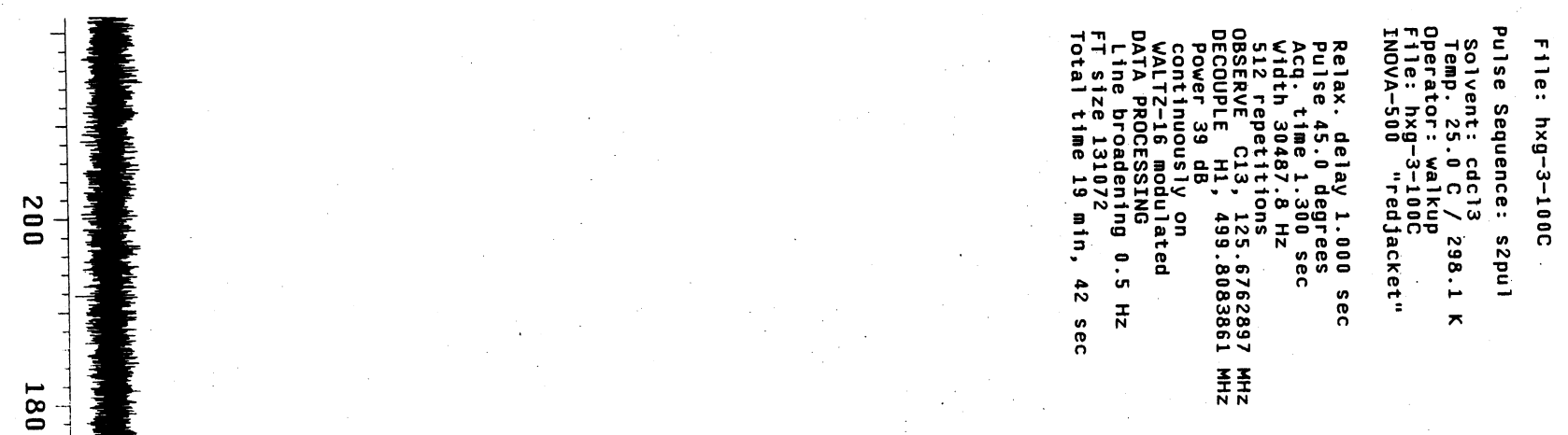




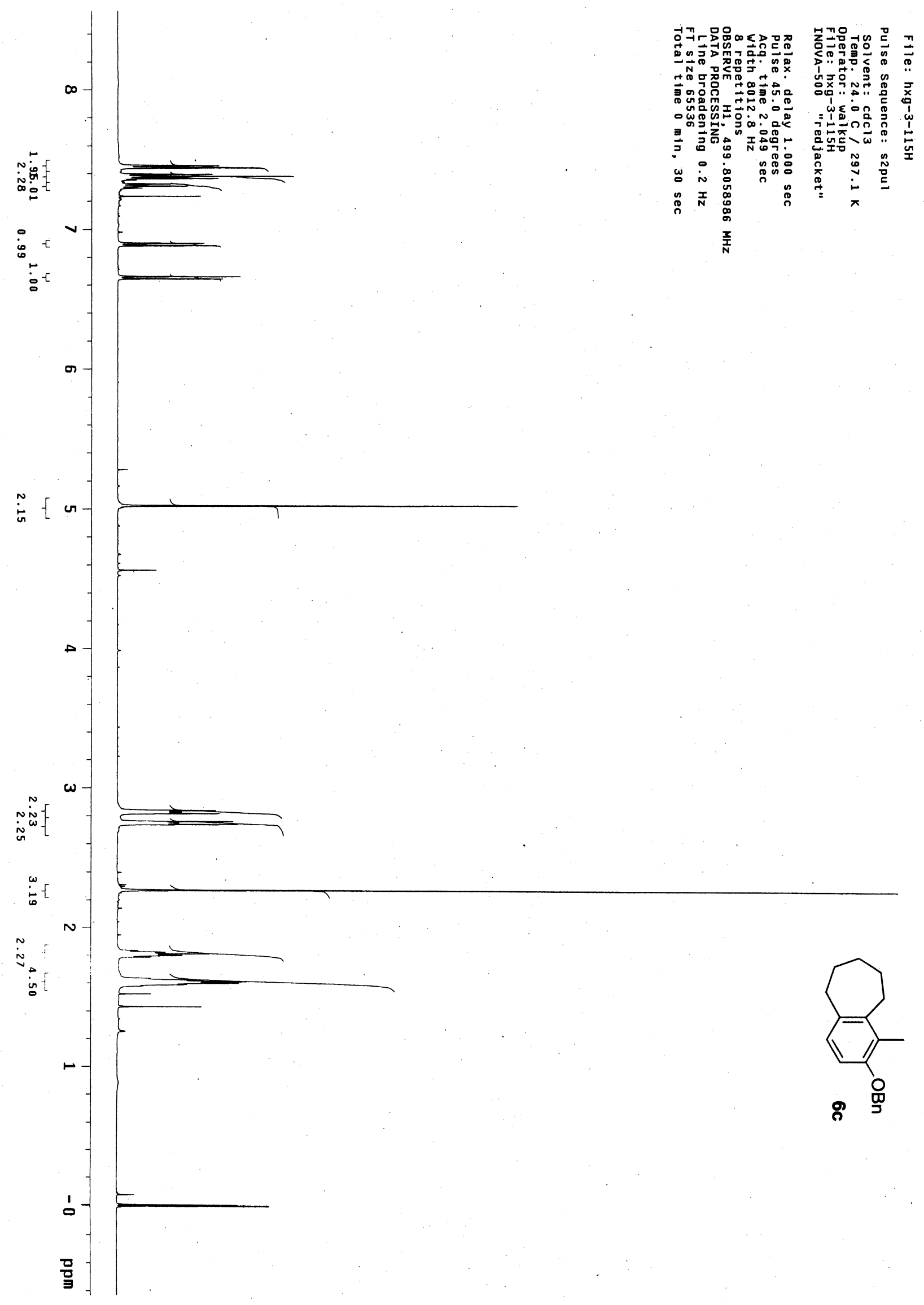



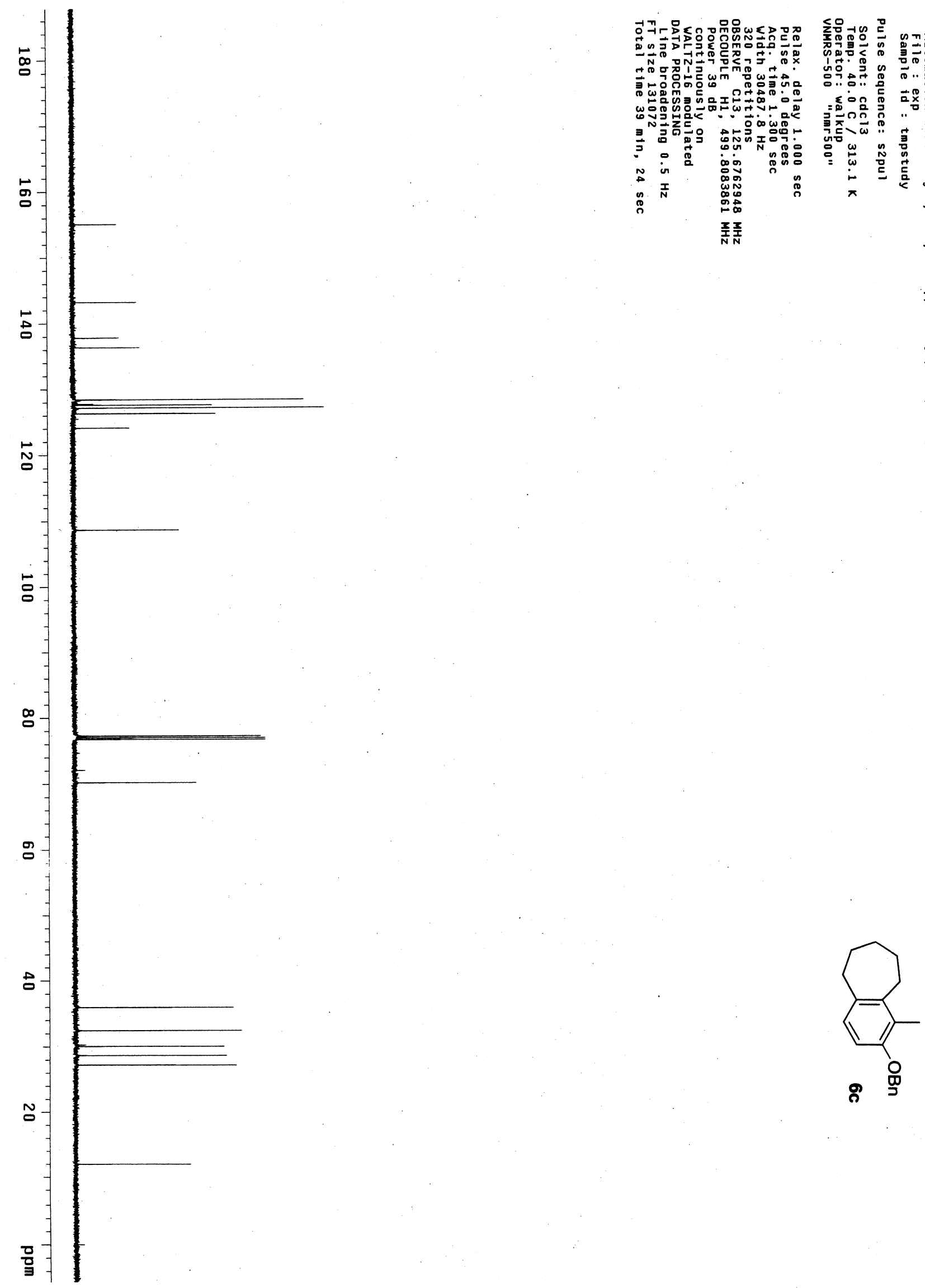


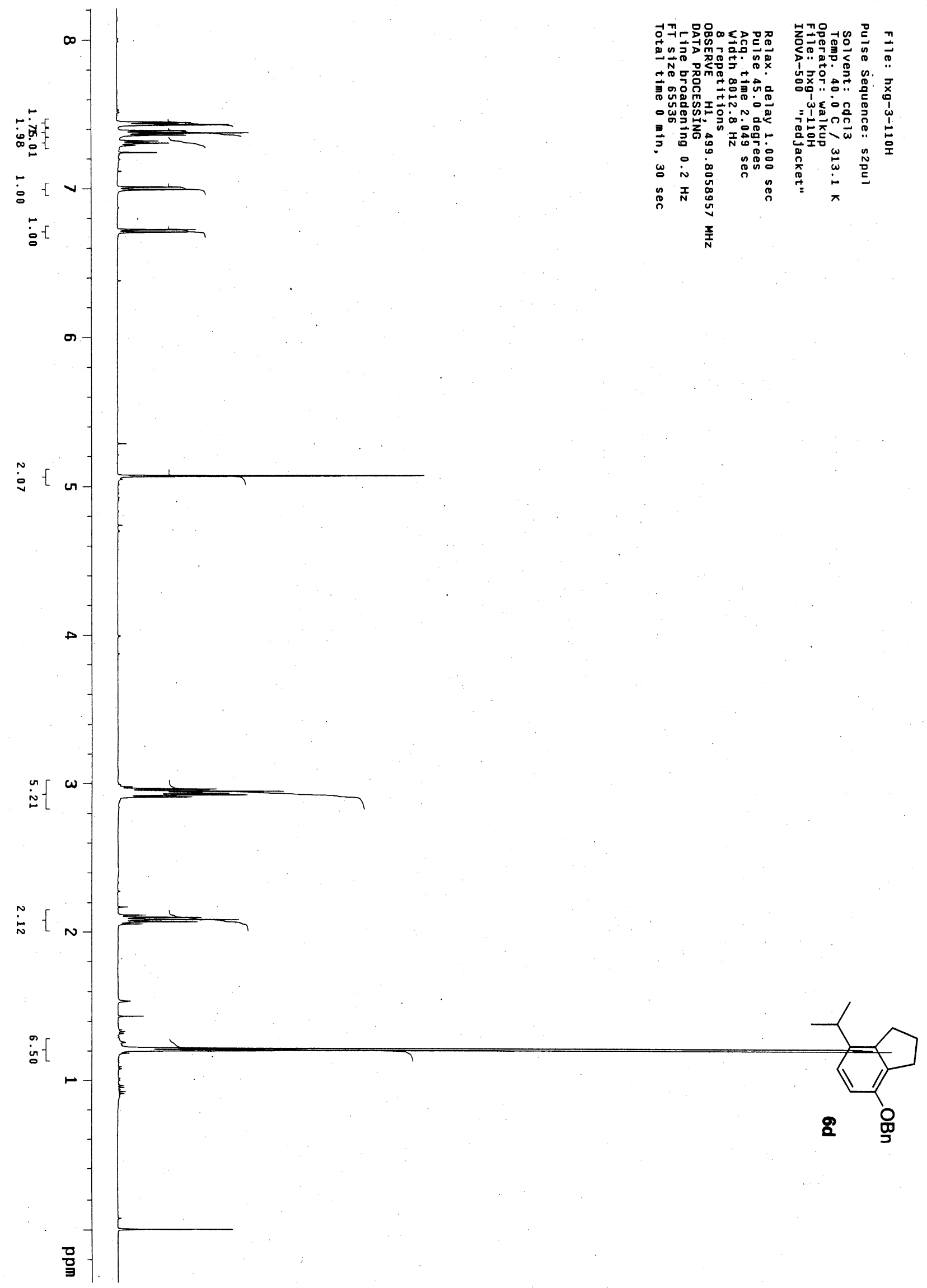



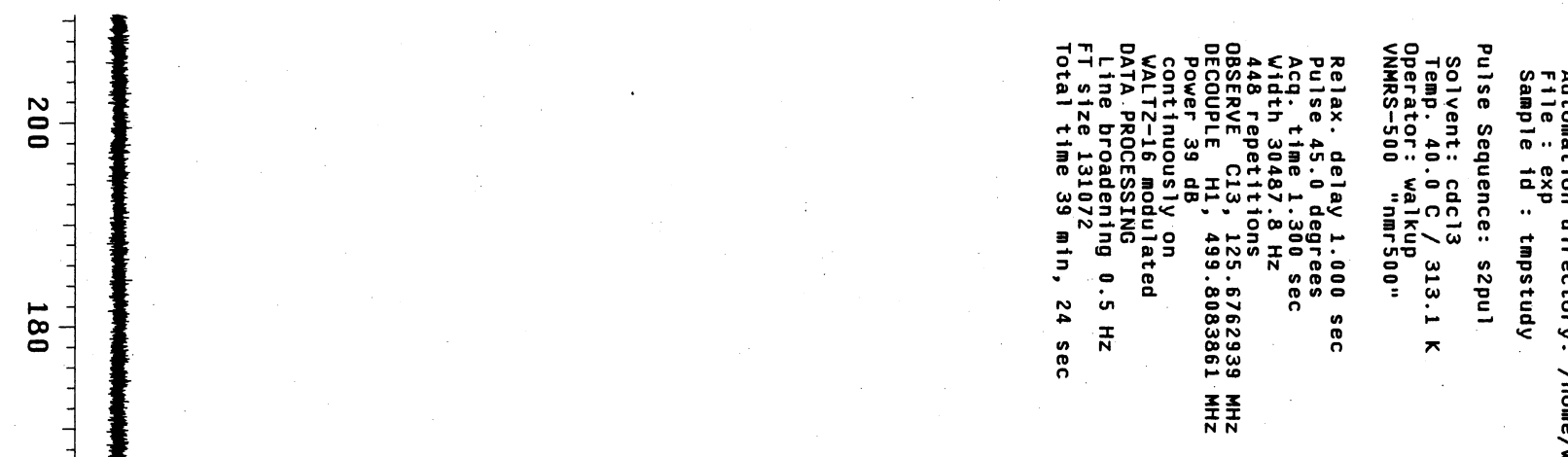

ס

$\stackrel{\leftarrow}{\circ}$

N

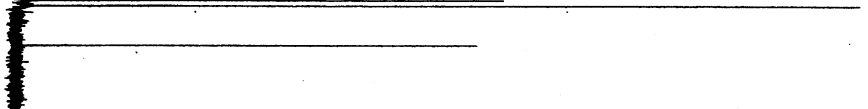

뭉

号

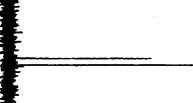




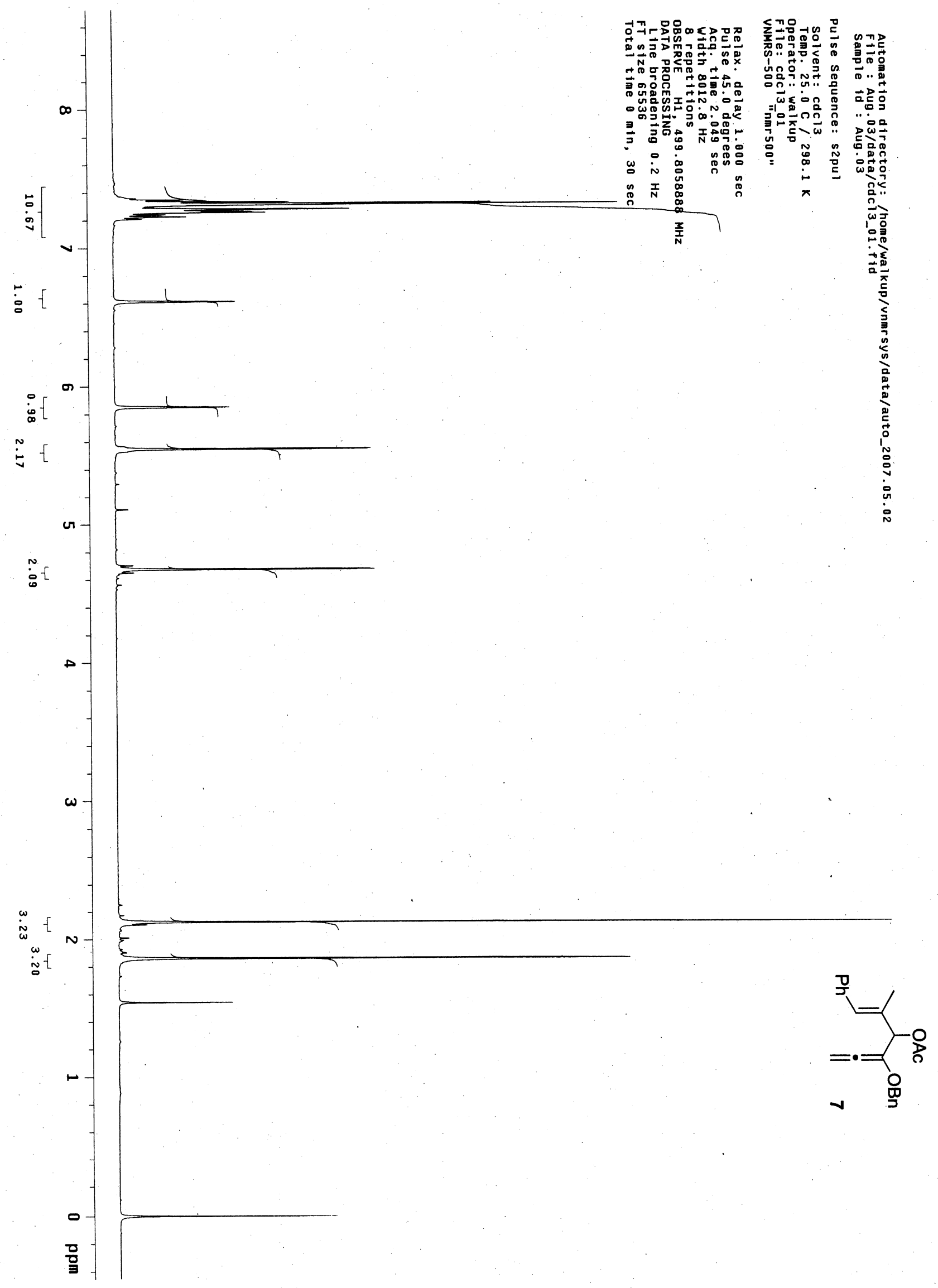




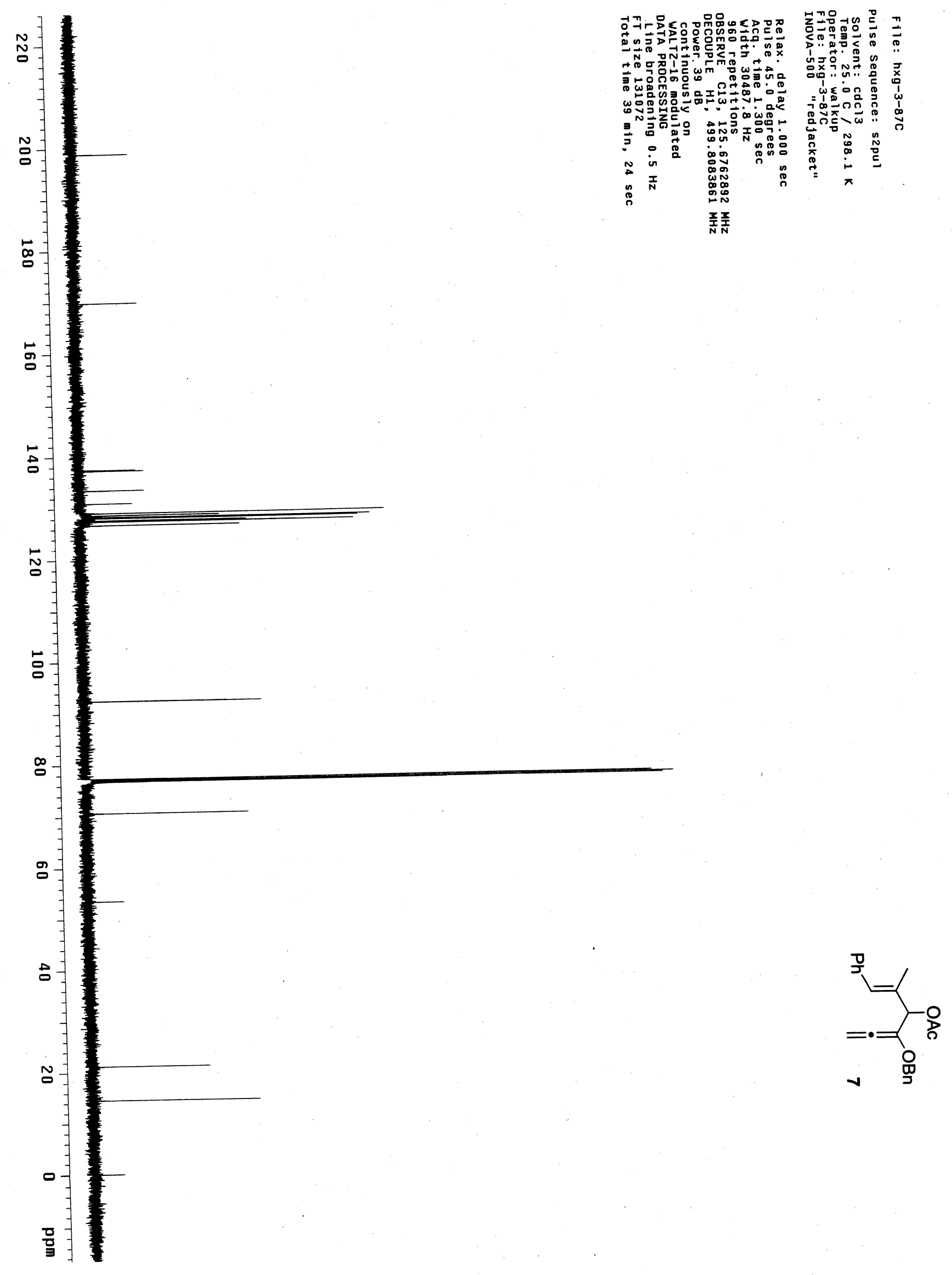




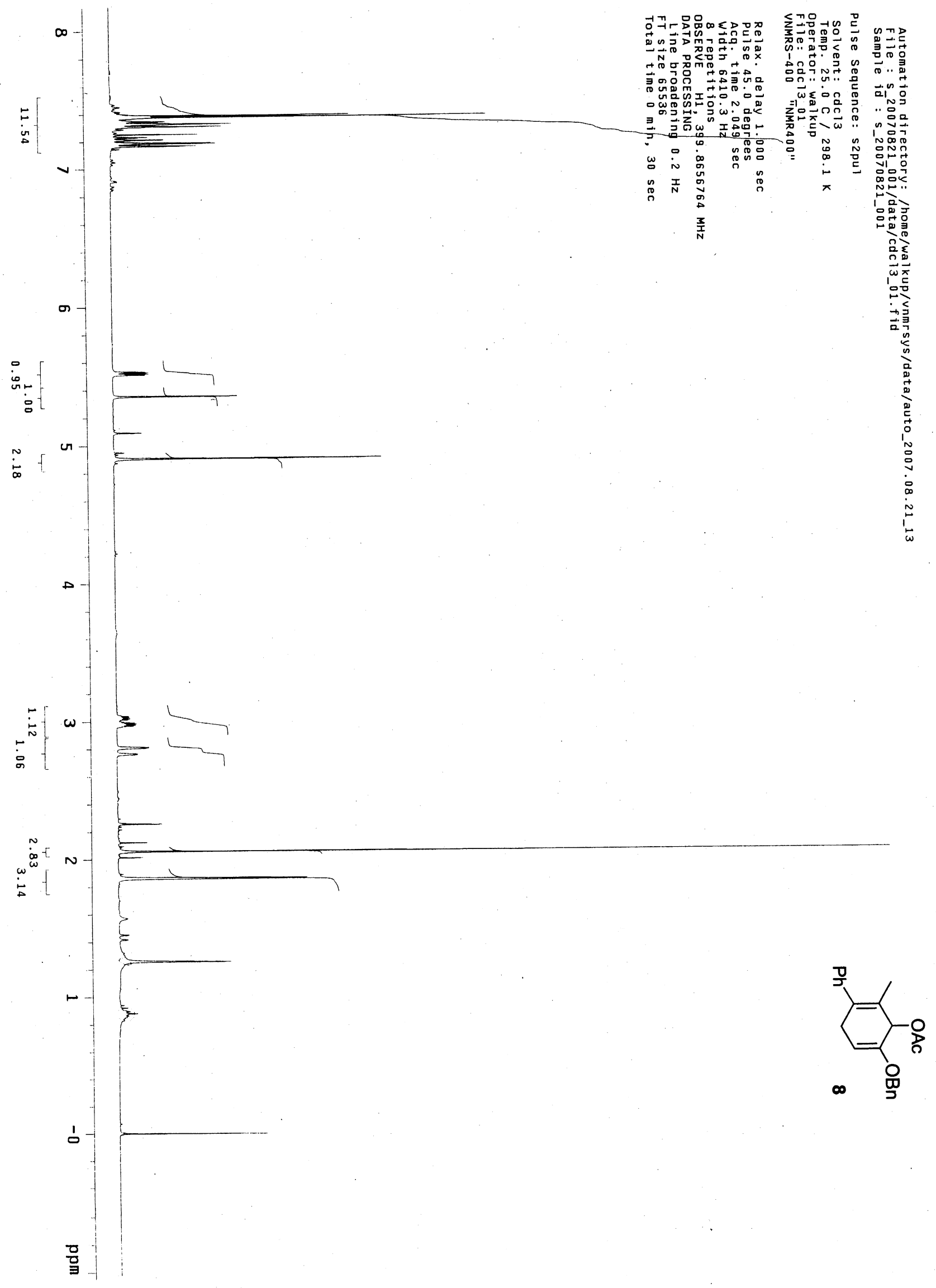



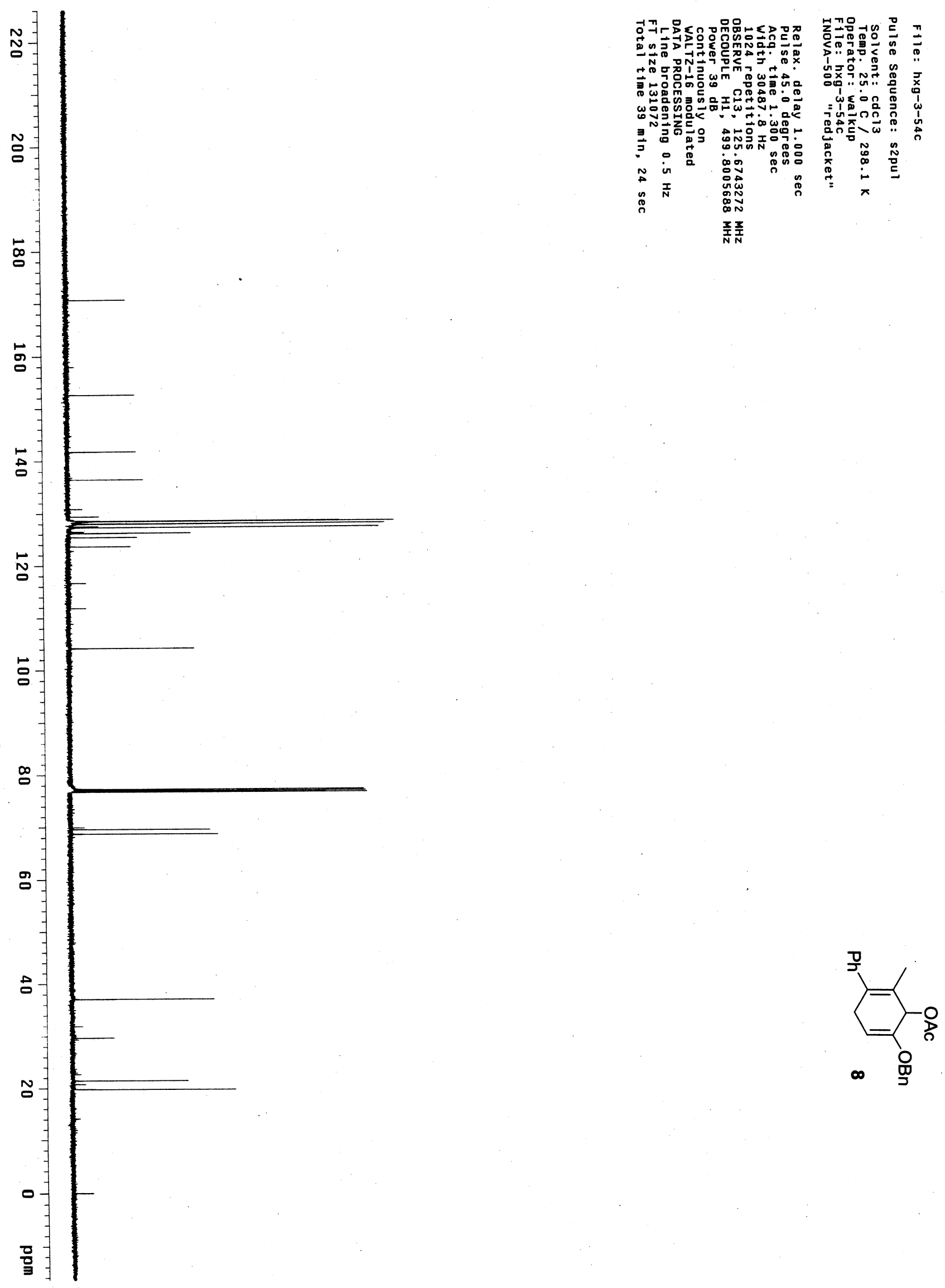

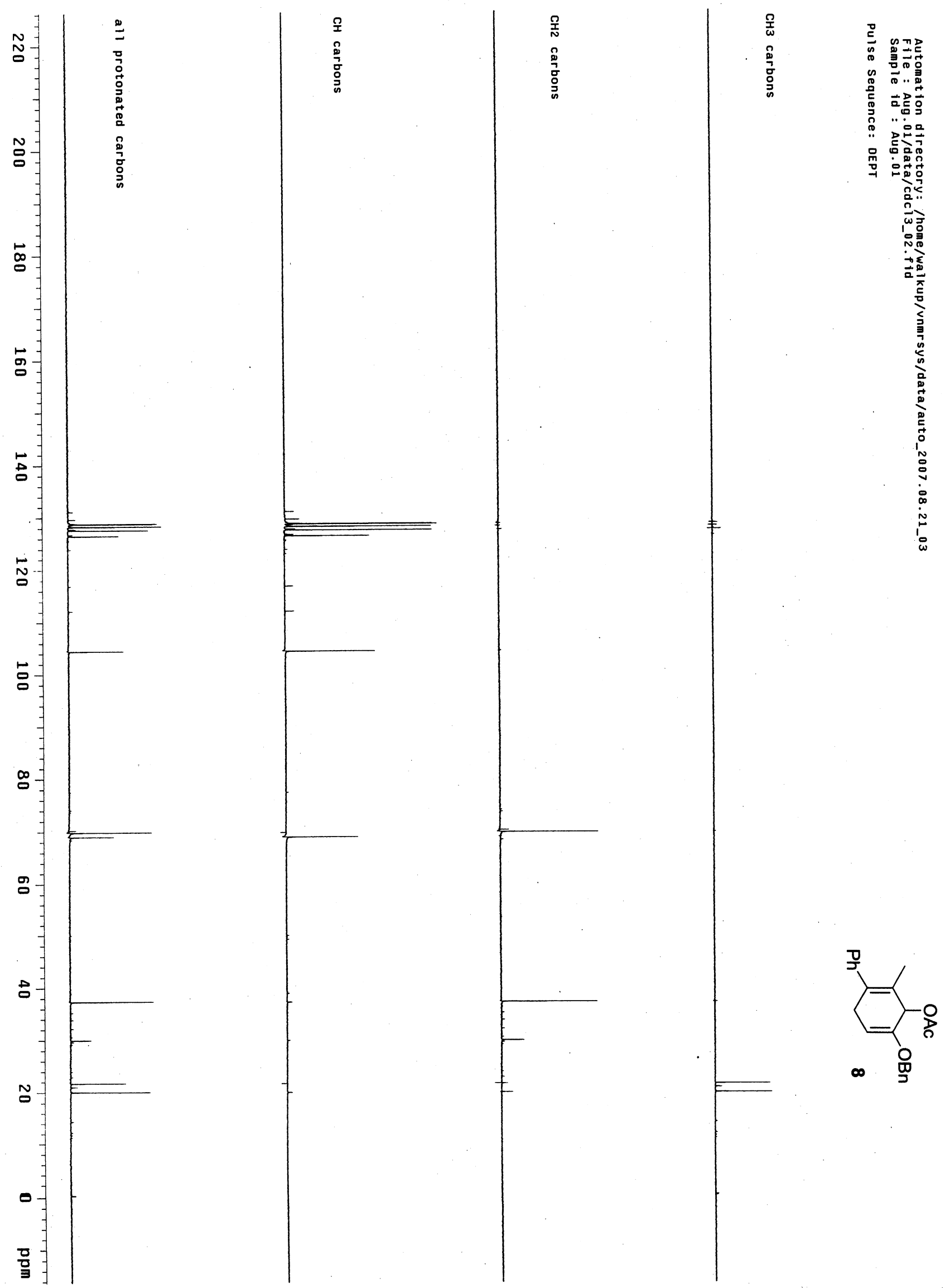

SI-59 

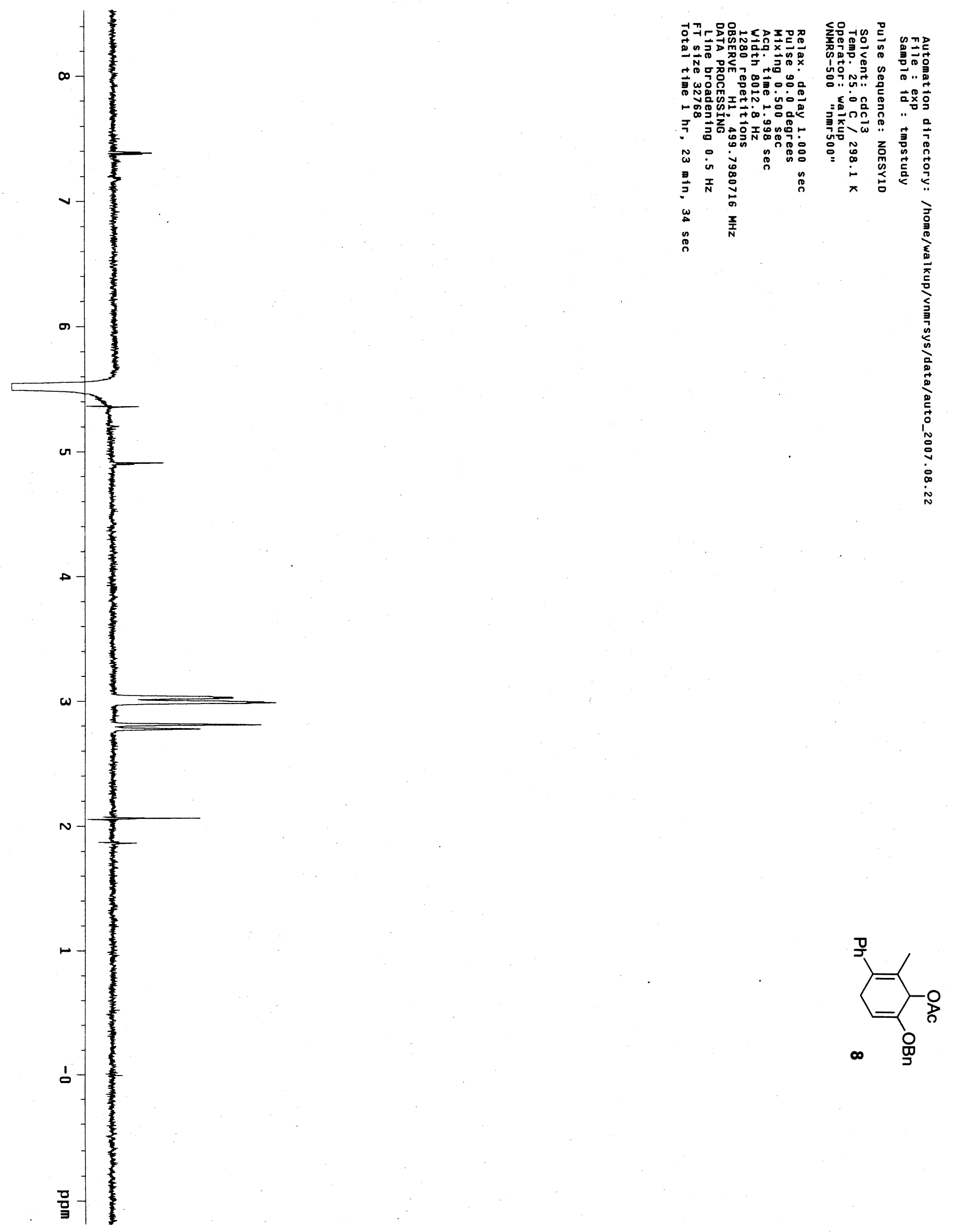

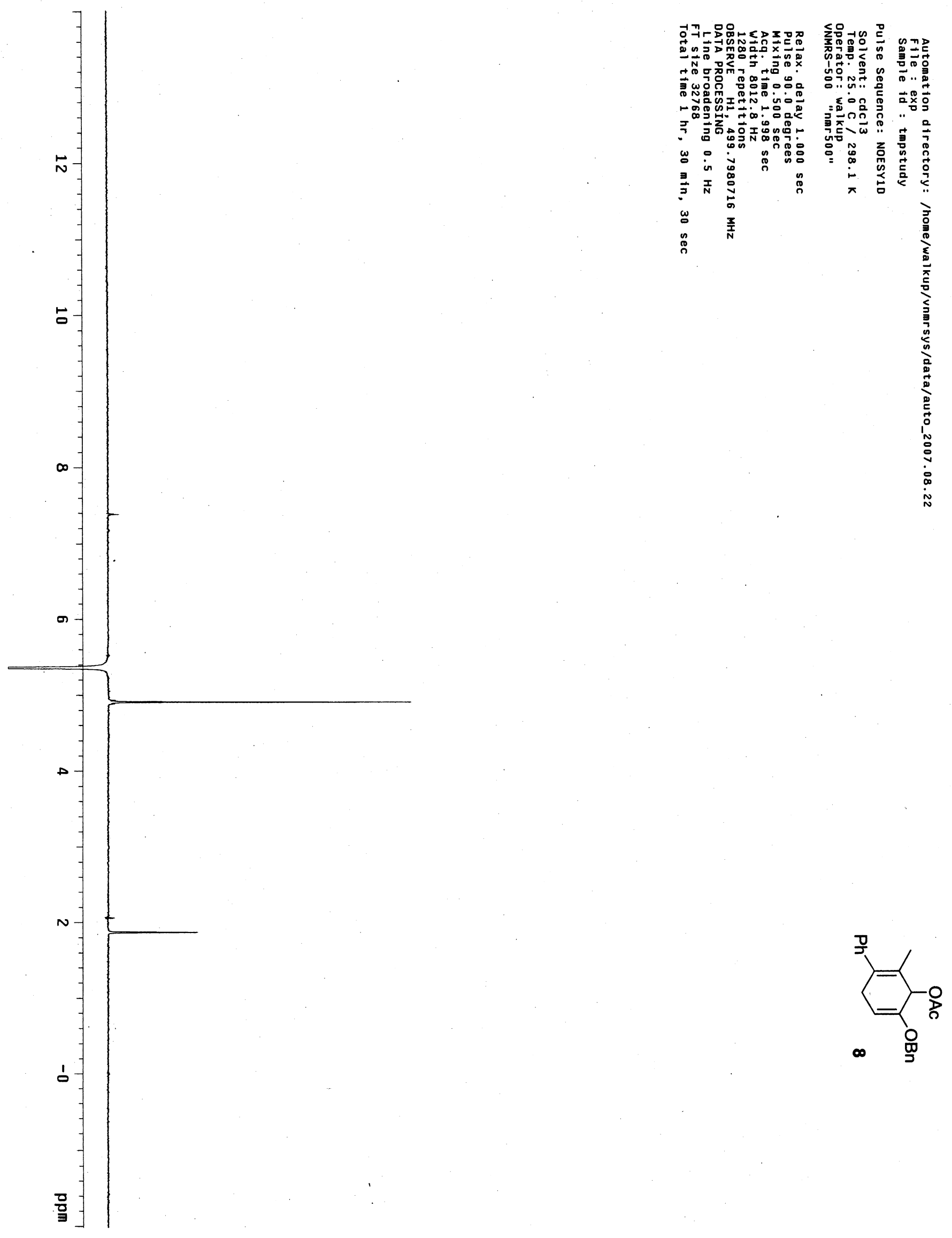


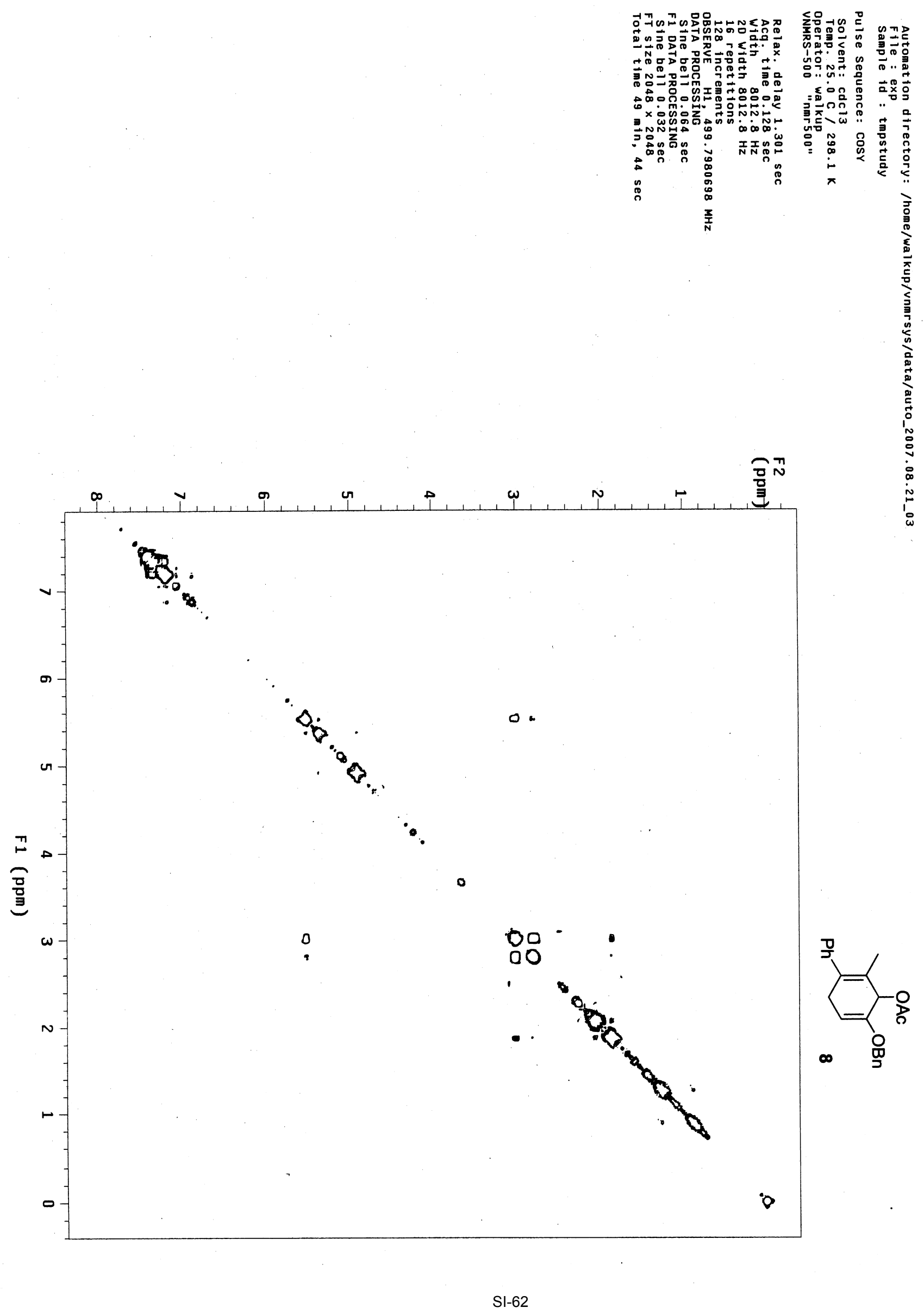



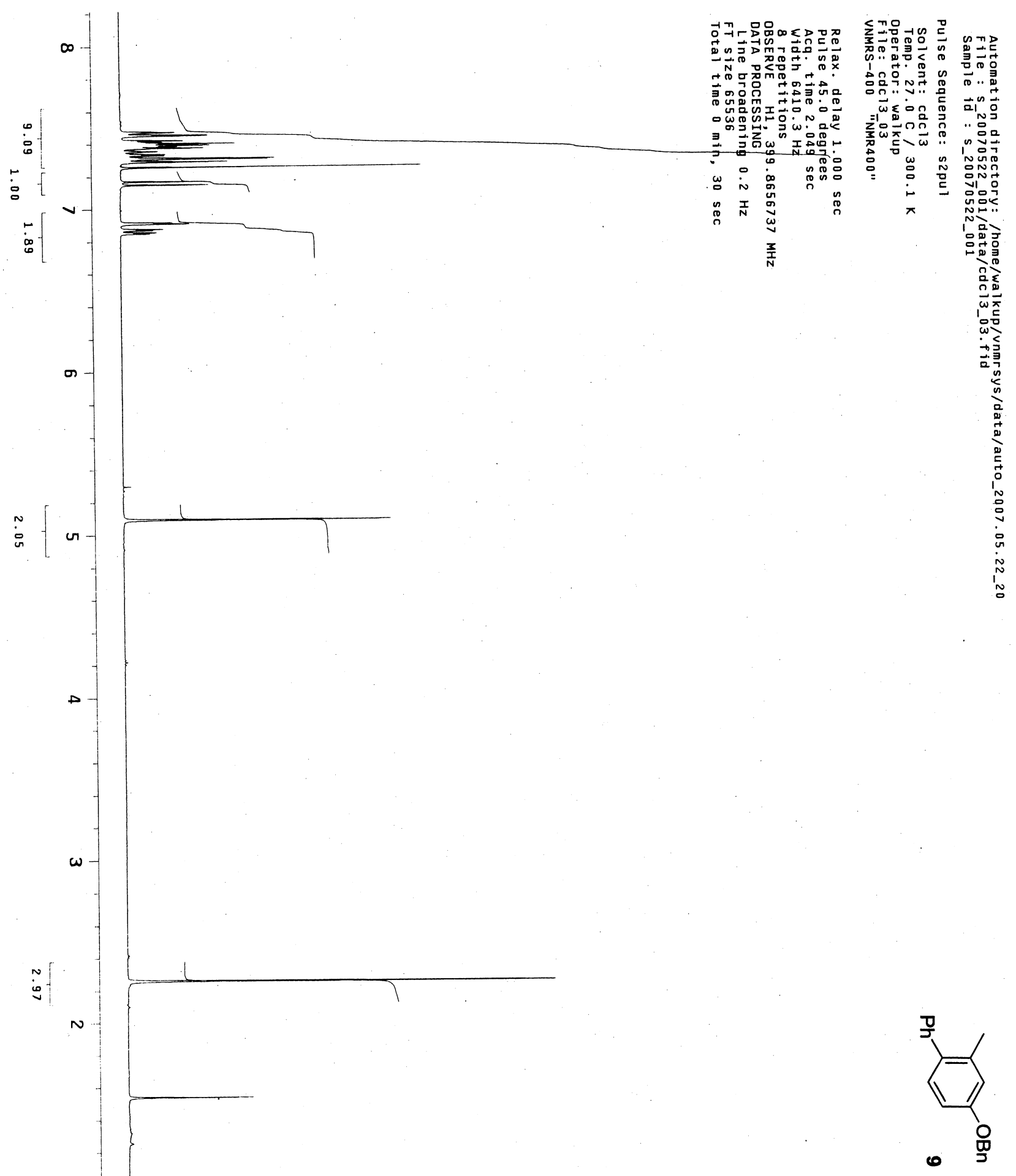

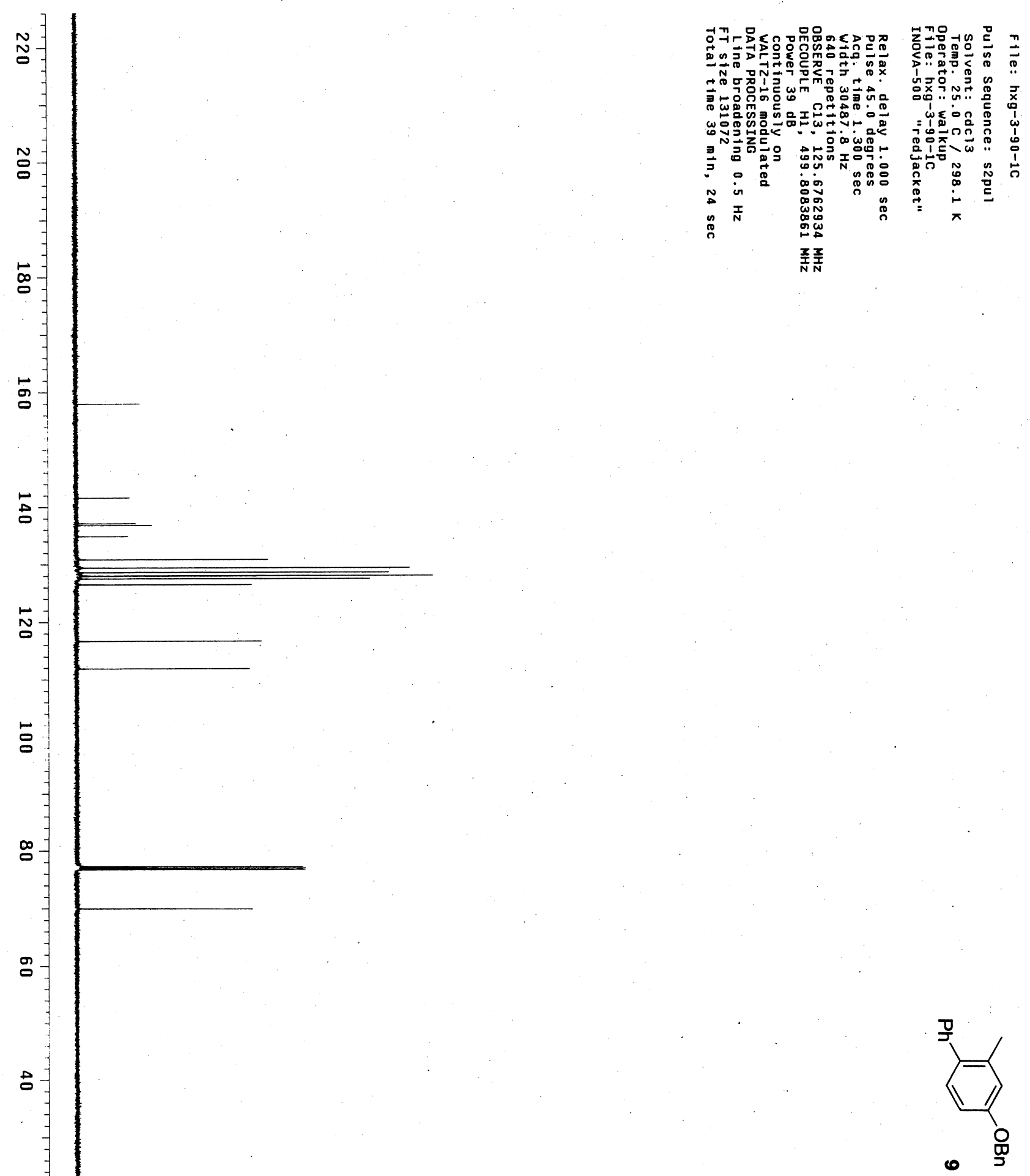